\title{
Contribuições ao Estudo de Modelos com Erros nas Variáveis
}

\author{
Mário de Castro Andrade Filho
}

TESE APRESENTADA

AO

INSTITUTO DE MATEMÁTICA E ESTATÍSTICA

DA

UNIVERSIDADE DE SÃO PAULO

PARA

OBTENÇÃO DO GRAU DE DOUTOR

EM

ESTATÍSTICA

Área de concentração: Estatística

Orientador: Prof. Dr. Heleno Bolfarine

Durante a elaboração deste trabalho o autor recebeu apoio financeiro da CAPES

-São Paulo, setembro de 2001- 


\title{
CONTRIBUIÇÕES AO ESTUDO DE MODELOS COM ERROS NAS VARIÁVEIS
}

\author{
Este exemplar corresponde à redação \\ final da tese de doutoramento, \\ devidamente corrigida e defendida por \\ Mário de Castro Andrade Filho e \\ aprovada pela comissão julgadora.
}

São Paulo, 5 de setembro de 2001.

Banca Examinadora:

Prof. Dr. Heleno Bolfarine - IME-USP

Prof. Dr. Julio da Motta Singer - IME-USP

Prof. ${ }^{a}$ Dr. ${ }^{a}$ Clarice Garcia Borges Demétrio - ESALQ-USP

Prof. Dr. Francisco Cribari Neto - UFPE

Prof. Dr. Manuel Jesus Galea Rojas - Universidad de Valparaíso 
À mamãe (IN MEMORIAM) Ao papai 


\section{Agradecimentos}

Ao Prof. Heleno Bolfarine, pela orientação deste trabalho, pelo estímulo e pelo companheirismo.

Ao Prof. Manuel Jesus Galea Rojas, pelas contribuições ao desenvolvimento deste trabalho.

À banca examinadora, pelos comentários e pelas sugestões.

Aos meus professores no IME-USP.

À turma de 1996 : Celso Cabral, Deborah Medeiros, Julio Hokama e Sebastião Lira.

À Eliana Zandonade, pela amizade lá e cá.

Aos professores e funcionários do Departamento de Estatística da UFES.

A todas as pessoas - distantes ou próximas, em Piripiri, em Fortaleza, no Rio de Janeiro, em Vila Velha, na Serra, em Vitória e em São Paulo - que participaram das várias etapas que compõem este trabalho. Felizmente, são muitas essas pessoas. 


\begin{abstract}
This work covers some errors-in-variables models. One of these models is the analysis of covariance (ANCOVA) model. The model considered encompasses both multiplicative and additive errors in the explanatory variables. Two consistent estimators of the treatment effects are compared in terms of asymptotic relative efficiency. It is shown that the naive estimator which ignores measurement errors is the one that presents the best behavior. A simulation study compares the relative merits of the estimators in small to moderate samples. In the sequence, the local influence approach for detecting the effect of small perturbations of the model or data is applied in the context of comparative calibration models. Such models are typically used for comparing several measuring instruments and can be considered in a functional version as well as in a structural version as is the case with ordinary errors-in-variables models. Different perturbation schemes are considered and two real data applications illustrate the usefulness of the approach. Finally, we extend the simple linear regression model to more general settings in which the explanatory variable is measured with error and there are two or more populations. Identifiability, estimation, a goodness-of-fit test and local influence are investigated. One set of real data is analyzed according to the proposed methods.
\end{abstract}




\section{Sumário}

1 Apresentação 12

2 Estimadores dos Efeitos dos Tratamentos em um Modelo ANCOVA com Erros nas Covariáveis $\quad 14$

2.1 Introdução . . . . . . . . . . . . . . . . . . . . . . 14

2.2 Modelo misto . . . . . . . . . . . . . . . . . . . . . . 15

2.3 Resultados assintóticos . . . . . . . . . . . . . . . . . . 17

2.4 Simulações . . . . . . . . . . . . . . . . . . . . . . 22

2.5 Extensões . . . . . . . . . . . . . . . . . . . . . 25

3 Influência Local no Modelo de Calibração Comparativa 27

3.1 Introdução . . . . . . . . . . . . . . . . . . . . . . . . . . . . . . . . .

3.2 O modelo homoscedástico . . . . . . . . . . . . . . . 30

3.2 .1 EMV . . . . . . . . . . . . . . . . . . 31

3.2 .2 Matriz de informação observada . . . . . . . . . . . . . 32

3.3 O modelo estrutural heteroscedástico . . . . . . . . . . . . . . 34

3.3 .1 EMV . . . . . . . . . . . . . . . . . . 35

3.3.2 Matriz de informação observada . . . . . . . . . . . . . . 37

3.4 Influência local . . . . . . . . . . . . . . . . . . . . 39

3.5 Esquemas de perturbação . . . . . . . . . . . . . . . . . . . 40

3.5.1 Perturbação da ponderação dos casos . . . . . . . . . . . . . . 41

Modelo homoscedástico . . . . . . . . . . . . . 41

Modelo estrutural heteroscedástico . . . . . . . . . . 42

3.5.2 Perturbação das observações . . . . . . . . . . . . . . . . . . . . 43

Modelo homoscedástico . . . . . . . . . . . . 43

Modelo estrutural heteroscedástico . . . . . . . . . 45 
3.5.3 Perturbação das variâncias dos erros de medição . . . . . . . . 46

3.6 Aplicações . . . . . . . . . . . . . . . . . . . . . . . . . . . . . . 49

4 Modelos Estruturais para Várias Populações $\quad 59$

4.1 Introdução . . . . . . . . . . . . . . . . . . . . . . . . . 59

4.2 Estimação . . . . . . . . . . . . . . . . . 62

4.2.1 Derivadas de segunda ordem . . . . . . . . . . . . . . 69

4.2 .2 Implementação computacional . . . . . . . . . . . . . . 76

4.3 Resultados assintóticos . . . . . . . . . . . . . . . . . 77

4.4 Influência local . . . . . . . . . . . . . . . . . . . . . . 79

4.4 .1 Perturbação das respostas . . . . . . . . . . . . . . . . . . . . . . . . . . 80

4.4.2 Perturbação das covariáveis . . . . . . . . . . . . . . . . . 81

4.5 Aplicação . . . . . . . . . . . . . . . . . . . . . . . 82

4.6 Comentários finais . . . . . . . . . . . . . . . . . . . 87

$\begin{array}{ll}\text { Referências Bibliográficas } & 88\end{array}$ 


\section{Lista de Tabelas}

2.1 Eficiências relativas assintóticas teóricas. . . . . . . . . . . . . . . 22 22

2.2 Valores dos parâmetros usados nas simulações. . . . . . . . . . . . . . . 23

2.3 Vícios simulados dos estimadores $\widehat{\tau}_{\mathrm{MQ}_{1}}, \widehat{\tau}_{c_{1}}$ e $\widehat{\tau}_{c_{1}}^{*} \ldots \ldots \ldots . . .24$

2.4 Eficiências relativas simuladas de $\widehat{\tau}_{\mathrm{MQ}_{1}}$ em relação a $\widehat{\tau}_{c_{1}}$ e $\widehat{\tau}_{c_{1}}^{*} \ldots \ldots .25$

3.1 Volume testicular $(\mathrm{em} \mathrm{ml})$ - Exemplo 3.1. . . . . . . . . . . . 51

4.1 Enumeração dos modelos estruturais entre vários grupos. . . . . . . . 61

4.2 Identificabilidade dos modelos estruturais entre vários grupos. . . . . 63 


\section{Lista de Figuras}

3.1 Algoritmo EM no modelo estrutural heteroscedástico. . . . . . . . . 36

3.2 Gráfico de índices de $\boldsymbol{l}_{\max }$ na perturbação da ponderação de casos Exemplo 3.1. . . . . . . . . . . . . . . . . . . 50 50

3.3 Gráficos do afastamento da verossimilhança $L D(\omega(a))$ versus a nas direções $\boldsymbol{l}_{\max }(+)$ e $\boldsymbol{e}_{20}(\square)$ - Exemplo 3.1. . . . . . . . . . 52

3.4 Gráfico de $\left|l_{\max }\right|$ versus a raiz cúbica das medições efetuadas com a técnica IV, perturbações aditivas - Exemplo 3.1. . . . . . . . . . 53

3.5 Gráfico de $\left|l_{\max }\right|$ versus a raiz cúbica das medições efetuadas com a técnica IV, perturbações multiplicativas - Exemplo 3.1 . . . . . . . 53

3.6 Gráficos do afastamento da verossimilhança $L D(\omega(a))$ versus a nas direções $\boldsymbol{l}_{\max }$ impondo perturbações aditivas $(+)$ e multiplicativas ( $\square$ ) nas medições feitas com a técnica IV - Exemplo 3.1. . . . . . . . . . 54

3.7 Gráfico de índices de $\boldsymbol{l}_{\max }$ na perturbação da ponderação de casos Exemplo 3.2. . . . . . . . . . . . . . . . . . . . 55

3.8 Gráfico de índices da distância de Cook $\left(D 2_{i}\right)$ - Exemplo 3.2. . . . . 56

3.9 Gráficos do afastamento da verossimilhança $L D_{1}(\omega(a))$ versus $a$ nas direções $l_{\max }(+)$ e $e_{67}(\square)$ - Exemplo 3.2. . . . . . . . . . . 57

3.10 Gráfico de índices de $\boldsymbol{l}_{\max }$ na perturbação da ponderação de casos, modelo heteroscedástico - Exemplo 3.2. . . . . . . . . . . 58

4.1 Algoritmo EM no modelo 34. . . . . . . . . . . . . 78

4.2 Gráfico de dispersão da largura e do comprimento de pétala e reta ajustada por mínimos quadrados - Exemplo 4.1. . . . . . . . 83

4.3 Gráfico de índices de $\boldsymbol{l}_{\max }$ nas perturbações aditiva (a) e multiplicativa. (b) das respostas, interesse em $\boldsymbol{\theta}$ - Exemplo 4.1. . . . . . . 85 
4.4 Gráfico de índices de $\boldsymbol{l}_{\max }$ nas perturbações aditiva (a) e multiplicativa (b) das respostas, interesse em $\theta_{1}$ - Exemplo 4.1. . . . . . . . 85

4.5 Gráfico de índices de $\boldsymbol{l}_{\max }$ nas perturbações aditiva (a) e multiplicativa (b) das covariáveis, interesse em $\boldsymbol{\theta}$ - Exemplo 4.1. . . . . . . . . 86

4.6 Gráfico de índices de $\boldsymbol{l}_{\max }$ nas perturbações aditiva (a) e multiplicativa (b) das covariáveis, interesse em $\theta_{1}$ - Exemplo 4.1. . . . . . . . 86 


\section{Capítulo 1}

\section{Apresentação}

Modelos com erros nas variáveis constituem o tema comum aos quatro capítulos deste trabalho. Genericamente, nesta classe de modelos algumas variáveis explicativas são inobserváveis exatamente. As observações são registradas na presença de erros de medição, sendo mais comum (e razoável em várias aplicações) uma estrutura aditiva e não-enviesada para eles, freqüentemente assumindo-se distribuição normal. Diversas variáveis nas áreas biomédica, agrícola, industrial e social, por exemplo, tipicamente são medidas com erros. Erros de medição têm efeitos importantes sobre as propriedades dos estimadores dos parâmetros dos modelos, de modo que técnicas desenvolvidas para corrigir estes efeitos recebem grande atenção por parte de pesquisadores.

A literatura sobre modelos com erros nas variáveis é vasta. Fuller (1987) é uma referência importante, basicamente voltada para modelos lineares. Brown \& Fuller (1990) reúnem trabalhos versando sobre assuntos como análise de resíduos, observações correlacionadas, modelos não-lineares, aspectos computacionais, robustez e um pouco de história. Modelos não-lineares são o principal interesse de Carroll, Ruppert \& Stefanski (1995). Recentemente, um volume tratando de modelos com erros nas variáveis foi adicionado à Kendall's Library of Statistics (Cheng \& Van Ness, 1999). Em Análise Numérica e Processamento de Sinais estuda-se um método chamado de mínimos quadrados totais, que está relacionado à modelagem com erros nas variáveis. Van Huffel \& Vandewalle (1991) expõem o método enfatizando pontos sobre a implementação computacional e dedicando um capítulo às propriedades estatísticas dos estimadores. Van Huffel (1997) traz contribuições de diversos autores nas áres de modelos com erros nas variáveis e mínimos quadrados totais. 
No segundo capítulo do nosso trabalho estudamos um modelo de análise de covariância (ANCOVA) em que temos variáveis explicativas medidas com erros aditivos e também variáveis explicativas contaminadas por erros multiplicativos. Comparamos dois estimadores consistentes dos efeitos dos tratamentos em termos de eficiência relativa assintótica. Um dos estimadores é o estimador de mínimos quadrados ordinários, cuja expressão depende do estimador de mínimos quadrados ordinários do vetor de coeficientes angulares (que é inconsistente). O outro estimador é uma modificação do estimador de mínimos quadrados ordinários em que utilizamos um estimador consistente para o vetor de coeficientes angulares, obtido sob certas suposições. Assintoticamente, o primeiro estimador é pelo menos tão eficiente quanto o segundo. Um estudo de simulação indica que esta conclusão parece válida também para amostras pequenas e moderadas. Portanto, estamos diante de um problema no qual um estimador que não leva em conta os erros de medição é preferível.

No Capítulo 3 aplicamos uma proposta de avaliação de influência local (Cook, 1986) ao modelo de calibração comparativa. Diferentes esquemas de perturbação do modelo postulado são considerados. O modelo de calibração comparativa descreve situações envolvendo formas distintas de medir uma mesma característica de interesse em um grupo de unidades experimentais. Embora este modelo seja um caso particular do modelo com erros nas variáveis multivariado, alguns esquemas de perturbação são específicos do problema de comparação comparativa e merecem estudo separado.

Uma relação linear envolvendo duas ou mais populações ocupa o último capítulo. Esta relação, dependendo da estrutura paramétrica, cobre vários modelos. Estendemos o modelo de regressão linear simples para situações mais gerais onde temos erros de medição na variável explicativa e diversas populações estão presentes. Discutimos a identificabilidade dos modelos. Apresentamos métodos de estimação dos parâmetros dos modelos, um teste de ajuste e uma análise de influência local (como no Capítulo 3).

Exemplos com dados reais ilustram as técnicas desenvolvidas nos capítulos 3 e 4 . Os programas computacionais que foram implementados podem ser obtidos enviando uma mensagem para o autor no endereço mario@cce.ufes.br. 


\section{Capítulo 2}

\section{Estimadores dos Efeitos dos Tratamentos em um Modelo ANCOVA com Erros nas Covariáveis}

\subsection{Introdução}

Análise de covariância (ANCOVA) é uma técnica que reúne elementos de análise de variância e regressão. Cochran (1957) e Cox \& McCullagh (1982) revisam seus aspectos históricos, características e principais usos. Este capítulo diz respeito ao modelo ANCOVA com erros de medição nas covariáveis. Um estudo de modelos com erros de medição é desenvolvido por Fuller (1987) e Cheng \& Van Ness (1999). Especificamente, Lord (1960), Porter (1967), Cochran (1968), DeGracie (1968), DeGracie \& Fuller (1972), Carroll, Gallo \& Gleser (1985), Carroll (1989) e Chen (1997) dedicam-se ao modelo ANCOVA com erros nas covariáveis.

Modelamos as covariáveis como variáveis aleatórias (modelo estrutural) distintas quanto à natureza dos erros de medição, algumas delas observadas com erros multiplicativos (Hwang, 1986) e as restantes observadas com erros aditivos (modelo misto). O objetivo principal é comparar - via eficiência relativa assintótica - dois estimadores consistentes dos efeitos dos tratamentos, derivados de dois estimadores dos coeficientes da regressão, um consistente e outro inconsistente. Os resultados aqui apresentados também se aplicam aos modelos aditivo e multiplicativo, casos 
particulares do modelo misto. Na Seção 2.4 em um estudo de simulação comparamos os estimadores propostos em amostras de tamanho pequeno a moderado. $\mathrm{O}$ material deste capítulo se fundamenta em Bolfarine \& de Castro (2000).

\subsection{Modelo misto}

Consideramos o modelo ANCOVA para dados colhidos sob um delineamento completamente casualizado e balanceado, efeitos dos tratamentos fixos e com $p$ covariáveis $(p \geq 1)$, expressável por

$$
Y_{i j}=\alpha+\tau_{i}+\boldsymbol{x}_{i j}^{\prime} \boldsymbol{\beta}+e_{i j}, \quad j=1, \ldots, n, i=1, \ldots, k,
$$

onde $Y_{i j}$ é o valor observado da resposta na $j$-ésima observação no $i$-ésimo tratamento, $\tau_{i}$ é o efeito do $i$-ésimo tratamento, $\beta=\left(\beta_{1}, \ldots, \beta_{p}\right)^{\prime}$ é o vetor de coeficientes da regressão das respostas nas covariáveis, $\boldsymbol{x}_{i j}=\left(x_{i j 1}, \ldots, x_{i j p}\right)^{\prime}, e_{i j}$ é a soma dos erros de medição e na equação, $k$ é o número de tratamentos e $n$ é o número de réplicas de cada tratamento. $\mathrm{O}$ vetor de efeitos dos tratamentos $\tau=\left(\tau_{1}, \ldots, \tau_{k}\right)^{\prime}$ está sujeito à restrição $\mathbf{1}_{k}^{\prime} \boldsymbol{\tau}=0$.

As $p$ covariáveis diferem quanto à natureza dos erros de medição. Subdividimos $\boldsymbol{x}_{i j}$ em dois grupos, o primeiro com $l$ e o segundo com $p-l$ componentes:

$$
\boldsymbol{x}_{i j}=\left(\boldsymbol{w}_{i j}^{\prime}, \boldsymbol{z}_{i j}^{\prime}\right)^{\prime}
$$

onde $\boldsymbol{w}_{i j}=\left(w_{i j 1}, \ldots, w_{i j l}\right)^{\prime}$ e $\boldsymbol{z}_{i j}=\left(z_{i j 1}, \ldots, z_{i j(p-l)}\right)^{\prime}$. Os valores de $\boldsymbol{x}_{i j}$ não são observáveis diretamente, mas com erros através de

$$
\mathbf{X}_{i j}=\left(\mathbf{W}_{i j}^{\prime}, \mathbf{Z}_{i j}^{\prime}\right)^{\prime}
$$

onde

$$
\mathbf{W}_{i j}=\boldsymbol{w}_{i j} \odot \boldsymbol{u}_{i j}, \mathbf{Z}_{i j}=z_{i j}+\boldsymbol{v}_{i j}
$$

e "๑" denota produto elemento a elemento (ou de Hadamard) (Styan, 1973). Seja $\mathrm{E}\left(\boldsymbol{x}_{i j}\right)=\boldsymbol{\mu}_{x}, \operatorname{com} \boldsymbol{\mu}_{x}=\left(\mathrm{E}\left(\boldsymbol{w}_{i j}^{\prime}\right), \mathrm{E}\left(\boldsymbol{z}_{i j}^{\prime}\right)\right)^{\prime}=\left(\boldsymbol{\mu}_{w}^{\prime}, \boldsymbol{\mu}_{z}^{\prime}\right)^{\prime}$. Fazendo $e_{. j}=\left(e_{1 j}, \ldots, e_{k j}\right)^{\prime}$, $\boldsymbol{u}_{. j}=\left(\boldsymbol{u}_{1 j}, \ldots, \boldsymbol{u}_{k j}\right)$ e definindo $\boldsymbol{v}_{. j}$ e $\boldsymbol{x}_{. j}$ de maneira similar, acrescentamos as suposições abaixo: 
S2.1

$$
\begin{gathered}
e_{i j}, \boldsymbol{u}_{i j}, \boldsymbol{v}_{i j} \text { e } \boldsymbol{x}_{i j} \text { são independentes, } \\
\left(e_{i j}, \boldsymbol{u}_{i j}^{\prime}, \boldsymbol{v}_{i j}^{\prime}, \boldsymbol{x}_{i j}^{\prime}\right)^{\prime} \stackrel{\text { iid }}{\sim}(\boldsymbol{\mu}, \boldsymbol{\Sigma}), j=1, \ldots, n, \quad i=1, \ldots, k,
\end{gathered}
$$

onde

$$
\boldsymbol{\mu}=\left(0, \mathbf{1}_{l}^{\prime}, 0_{p-l}^{\prime}, \boldsymbol{\mu}_{x}^{\prime}\right)^{\prime} \text { e } \Sigma=\operatorname{bdiag}\left(\sigma_{e e}, \Sigma_{u u}, \Sigma_{v v}, \Sigma_{x x}\right)
$$

com

$$
\Sigma_{x x}=\left(\begin{array}{cc}
\Sigma_{w w} & \Sigma_{w z} \\
& \Sigma_{z z}
\end{array}\right) \text { definida positiva }
$$

ou, considerando todos os tratamentos,

$$
\operatorname{vec}\left(\begin{array}{l}
e_{. j}^{\prime} \\
\boldsymbol{u}_{. j} \\
\boldsymbol{v}_{. j} \\
\boldsymbol{x}_{. j}
\end{array}\right) \stackrel{\text { iid }}{\sim}\left(\mathbf{1}_{k} \otimes \boldsymbol{\mu}, \mathbf{I}_{k} \otimes \Sigma\right), j=1, \ldots, n .
$$

Em S2.1 «iid $\sim(\mathbf{m}, \mathbf{M})$ ” denota vetores independentes e identicamente distribuídos com média m e matriz de covariâncias $\mathbf{M}$, "bdiag" é uma matriz diagonal em blocos constituída dos blocos indicados e "vec(M)" é o vetor formado pelas colunas de $\mathbf{M}$. Em matrizes simétricas omitimos o bloco inferior esquerdo. A aleatorização permite verificar se a suposição $\boldsymbol{x}_{i j} \stackrel{\text { iid }}{\sim}\left(\boldsymbol{\mu}_{x}, \boldsymbol{\Sigma}_{x x}\right)$ é justificável. As matrizes $\boldsymbol{\Sigma}_{u u}$ e $\boldsymbol{\Sigma}_{v v}$ podem ser singulares; em particular, podemos ter covariáveis medidas sem erro.

As médias das observações, na notação ponto e barra usual, são calculadas conforme abaixo:

$$
\begin{gathered}
\overline{\mathbf{X}}_{.}=\left(\overline{\mathbf{X}}_{1 .}, \ldots, \overline{\mathbf{X}}_{k .}\right), \overline{\mathbf{X}}_{i .}=\frac{1}{n} \sum_{j=1}^{n} \mathbf{X}_{i j}, i=1, \ldots, k, \\
\overline{\mathbf{X}}_{. .}=\frac{1}{k} \sum_{i=1}^{k} \overline{\mathbf{X}}_{i .}=\frac{1}{k} \overline{\mathbf{X}}_{.} \mathbf{1}_{k}, \\
\overline{\mathbf{Y}}_{.}=\left(\bar{Y}_{1 .}, \ldots, \bar{Y}_{k .}\right)^{\prime}, \bar{Y}_{i .}=\frac{1}{n} \sum_{j=1}^{n} Y_{i j}, i=1, \ldots, k, \\
\bar{Y}_{. .}=\frac{1}{k} \sum_{i=1}^{k} \bar{Y}_{i .}=\frac{1}{k} \mathbf{1}_{k}^{\prime} \overline{\mathbf{Y}}_{.} .
\end{gathered}
$$


Denotamos por $\widehat{\boldsymbol{\beta}}_{g}$ uma seqüencia de estimadores de $\boldsymbol{\beta}$ tal que $\sqrt{n}\left(\widehat{\boldsymbol{\beta}}_{g}-\mathbf{B} \boldsymbol{\beta}\right)=$ $O_{p}(\mathbf{1})$, onde $\mathbf{B}$ é uma matriz $p \times p$. As ordens estocásticas $O_{p}(1)$ e $o_{p}(1)$ encontram-se definidas em Sen \& Singer (1993, definições 2.2.5 e 2.2.7). Baseando-se no estimador de mínimos quadrados ordinários, uma classe de estimadores para o vetor de efeitos dos tratamentos é dada por

$$
\begin{aligned}
\widehat{\boldsymbol{\tau}}_{g} & =\left(\overline{\mathbf{Y}}_{.}-\mathbf{1}_{k} \bar{Y}_{. .}\right)-\left(\overline{\mathbf{X}}_{.}^{\prime}-\mathbf{1}_{k} \overline{\mathbf{X}}_{. .}^{\prime}\right) \widehat{\boldsymbol{\beta}}_{g} \\
& =\mathrm{C}_{k}\left(\overline{\mathbf{Y}}_{.}-\overline{\mathbf{X}}_{.}^{\prime} \widehat{\boldsymbol{\beta}}_{g}\right)
\end{aligned}
$$

onde $\mathbf{C}_{k}=\mathbf{I}_{k}-\frac{1}{k} \mathbf{1}_{k} \mathbf{1}_{k}^{\prime}$ é a matriz de centralização.

\subsection{Resultados assintóticos}

O próximo resultado fornece a distribuição assintótica do estimador $\widehat{\tau}_{g}$.

Teorema 2.1 No modelo (2.1)-(2.3) com S2.1, e supondo que $\sqrt{n}\left(\widehat{\boldsymbol{\beta}}_{g}-\mathbf{B} \boldsymbol{\beta}\right)=$ $O_{p}(1)$ temos

$$
\sqrt{n}\left(\widehat{\tau}_{g}-\boldsymbol{\tau}\right) \stackrel{\mathrm{d}}{\longrightarrow} \mathrm{N}_{k}\left(\mathbf{0}, \mathbf{C}_{k}\left(\sigma_{e e}+\beta^{\prime} \Sigma_{x x} \boldsymbol{\beta}-2 \boldsymbol{\beta}^{\prime} \mathbf{B}^{\prime} \Sigma_{x x} \beta+\boldsymbol{\beta}^{\prime} \mathbf{B}^{\prime} \Sigma_{X X} \mathbf{B} \boldsymbol{\beta}\right)\right)
$$

quando $n \rightarrow \infty$, onde

$$
\begin{aligned}
& \Sigma_{X X}=\Sigma_{x x}+\Sigma_{1}, \\
& \Sigma_{1}=\left(\begin{array}{cc}
\Sigma_{u u}^{*} & 0 \\
& \Sigma_{v v}
\end{array}\right)
\end{aligned}
$$

com

$$
\Sigma_{u u}^{*}=\Sigma_{u u} \odot\left(\Sigma_{w w}+\boldsymbol{\mu}_{w} \boldsymbol{\mu}_{w}^{\prime}\right)
$$

Prova. De (2.1) obtemos $\overline{\mathbf{Y}} .=\mathbf{1}_{k} \alpha+\boldsymbol{\tau}+\overline{\boldsymbol{x}}^{\prime} \boldsymbol{\beta}+\overline{\boldsymbol{e}}_{\text {. }}$, onde $\overline{\boldsymbol{x}}$. e $\overline{\boldsymbol{e}}$. são definidas analogamente a $\overline{\mathrm{X}}$. e $\overline{\mathrm{Y}}$. Substituindo $\overline{\mathrm{Y}}$. acima em (2.5), algumas passagens conduzem a.

$$
\sqrt{n}\left(\widehat{\boldsymbol{\tau}}_{g}-\tau\right)=\mathrm{C}_{k} \overline{\mathrm{X}}^{\prime} \sqrt{n}\left(\mathrm{~B} \boldsymbol{\beta}-\widehat{\boldsymbol{\beta}}_{g}\right)+\left[\mathrm{C}_{k} \otimes\left(1, \boldsymbol{\beta}^{\prime},-\boldsymbol{\beta}^{\prime} \mathrm{B}^{\prime}\right)\right] \sqrt{n} \operatorname{vec}\left(\begin{array}{l}
\bar{e}^{\prime} \\
\overline{\boldsymbol{x}} \\
\overline{\mathrm{X}}
\end{array}\right)
$$


As suposições S2.1 e (2.1)-(2.3) implicam que

$$
\operatorname{vec}\left(\begin{array}{c}
e_{. j}^{\prime} \\
x_{. j} \\
\mathbf{X}_{. j}
\end{array}\right) \stackrel{\text { iid }}{\sim}\left(\mathbf{1}_{k} \otimes \boldsymbol{\mu}^{*}, \mathbf{I}_{k} \otimes \Sigma^{*}\right), \quad j=1, \ldots, n
$$

com

$$
\mathbf{X}_{. j}=\left(\mathbf{X}_{1 j}, \ldots, \mathbf{X}_{k j}\right), \quad \boldsymbol{\mu}^{*}=\left(0, \boldsymbol{\mu}_{x}^{\prime}, \boldsymbol{\mu}_{x}^{\prime}\right)^{\prime} \text { e } \quad \boldsymbol{\Sigma}^{*}=\left(\begin{array}{ccc}
\sigma_{e e} & 0 & 0 \\
& \Sigma_{x x} & \boldsymbol{\Sigma}_{x x} \\
& & \Sigma_{X X}
\end{array}\right) .
$$

Levando em conta que $\left[\mathbf{C}_{k} \otimes\left(1, \boldsymbol{\beta}^{\prime},-\boldsymbol{\beta}^{\prime} \mathbf{B}^{\prime}\right)\right]\left(\mathbf{1}_{k} \otimes \boldsymbol{\mu}^{*}\right)=0$ segue que

$$
\begin{aligned}
\sqrt{n}\left(\widehat{\tau}_{g}-\tau\right)= & \mathbf{C}_{k} \overline{\mathbf{X}}_{.}^{\prime} \sqrt{n}\left(\mathbf{B} \boldsymbol{\beta}-\widehat{\boldsymbol{\beta}}_{g}\right) \\
& +\left[\mathbf{C}_{k} \otimes\left(1, \boldsymbol{\beta}^{\prime},-\boldsymbol{\beta}^{\prime} \mathbf{B}^{\prime}\right)\right] \sqrt{n}\left[\operatorname{vec}\left(\begin{array}{c}
\bar{e}^{\prime} \\
\overline{\boldsymbol{x}} . \\
\overline{\mathbf{X}} .
\end{array}\right)-\mathbf{1}_{k} \otimes \boldsymbol{\mu}^{*}\right]
\end{aligned}
$$

Os vetores $\operatorname{vec}\left(\mathbf{X}_{. j}\right), j=1, \ldots, n$, formam uma seqüência de vetores aleatórios iid com vetor de médias $\mathbf{1}_{k} \otimes \boldsymbol{\mu}_{x}$. A lei forte dos grandes números (Sen \& Singer, 1993, Teorema 2.3.13) implica que $\overline{\mathbf{X}} . \stackrel{\text { qc }}{\longrightarrow} \mathbf{1}_{k}^{\prime} \otimes \boldsymbol{\mu}_{x}$, quando $n \rightarrow \infty$. Conseqüentemente, $\mathrm{C}_{k} \overline{\mathbf{X}}^{\prime} \stackrel{\mathrm{qc}}{\longrightarrow}\left(\mathbf{I}_{k}-\frac{1}{k} \mathbf{1}_{k} \mathbf{1}_{k}^{\prime}\right) \mathbf{1}_{k} \boldsymbol{\mu}_{x}^{\prime}=\mathbf{0} \mathrm{e}$

$$
\mathrm{C}_{k} \overline{\mathrm{X}}^{\prime} \cdot \sqrt{n}\left(\mathbf{B} \boldsymbol{\beta}-\widehat{\boldsymbol{\beta}}_{g}\right)=o_{p}(\mathbf{1}) .
$$

O teorema central do limite (Muirhead, 1982, Teorema 1.2.16) aplicado à seqüência (2.8) fornece

$$
\sqrt{n}\left[\operatorname{vec}\left(\begin{array}{l}
\bar{e}^{\prime} \\
\overline{x_{.}} \\
\overline{\mathbf{X}} .
\end{array}\right)-\mathbf{1}_{k} \otimes \boldsymbol{\mu}^{*}\right] \stackrel{\mathrm{d}}{\longrightarrow} \mathrm{N}_{k}\left(\mathbf{0}, \mathbf{I}_{k} \otimes \Sigma^{*}\right) .
$$

Portanto, a segunda parcela em (2.9) é assintoticamente distribuída segundo a distribuição

$$
\mathrm{N}_{k}\left(\mathbf{0}, \mathrm{C}_{k}\left(\sigma_{e e}+\beta^{\prime} \Sigma_{x x} \beta-2 \beta^{\prime} \mathbf{B}^{\prime} \Sigma_{x x} \beta+\beta^{\prime} \mathbf{B}^{\prime} \Sigma_{X X} \mathbf{B} \beta\right)\right) .
$$

Este resultado, (2.10) e uma aplicação do teorema de Slutsky (Sen \& Singer, 1993, Teorema 3.4.3) em (2.9) completam a prova. 
Em seguida consideramos estimadores de $\beta$, a partir dos quais derivamos estimadores de $\boldsymbol{\tau}$ através de (2.5). Sejam $\mathbf{S}_{X X}$ e $s_{X Y}$ as covariâncias amostrais entre as covariáveis e entre estas e a variável resposta, respectivamente, tendo expressões

$$
\begin{gathered}
\mathbf{S}_{X X}=\frac{1}{k(n-1)} \sum_{i=1}^{k} \sum_{j=1}^{n}\left(\mathbf{X}_{i j}-\overline{\mathbf{X}}_{i .}\right)\left(\mathbf{X}_{i j}-\overline{\mathbf{X}}_{i .}\right)^{\prime} \mathrm{e} \\
s_{X Y}=\frac{1}{k(n-1)} \sum_{i=1}^{k} \sum_{j=1}^{n}\left(\mathbf{X}_{i j}-\overline{\mathbf{X}}_{i .}\right)\left(Y_{i j}-\bar{Y}_{i .}\right) .
\end{gathered}
$$

Assumindo $\mathbf{S}_{X X}$ inversível, dispomos do estimador de mínimos quadrados de $\beta$, que ignora os erros de medição (estimador ingênuo), cuja expressão é

$$
\widehat{\boldsymbol{\beta}}_{\mathrm{MQ}}=\mathrm{S}_{X X}^{-1} s_{X Y} .
$$

A consistência deste estimador pode ser analisada sob S2.1. Aplicando a lei forte dos grandes números a (2.11), temos que $\mathbf{S}_{X X} \stackrel{\mathrm{qc}}{\longrightarrow} \Sigma_{X X}=\boldsymbol{\Sigma}_{x x}+\boldsymbol{\Sigma}_{1}$ e $\boldsymbol{s}_{X Y} \stackrel{\mathrm{qc}}{\longrightarrow} \boldsymbol{\Sigma}_{X Y}=$ $\Sigma_{x x} \beta$. Logo,

$$
\widehat{\boldsymbol{\beta}}_{\mathrm{MQ}} \stackrel{\mathrm{qc}}{\longrightarrow} \Sigma_{X X}^{-1} \sigma_{X Y}=\left(\Sigma_{x x}+\Sigma_{1}\right)^{-1} \Sigma_{x x} \beta,
$$

quando $n \rightarrow \infty$, mostrando a inconsistência de $\widehat{\boldsymbol{\beta}}_{\mathrm{MQ}}$. Isto caracteriza um tipo de atenuação (Fuller, 1987) em que

$$
\left\|\Sigma_{X X}^{-1} \Sigma_{x x} \beta\right\| \leq\|\beta\|,
$$

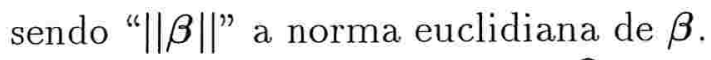

Se tivermos um estimador $\widehat{\Sigma_{1}}$ de $\Sigma_{1}$ satisfazendo $\widehat{\Sigma_{1}} \stackrel{\text { qc }}{\longrightarrow} \Sigma_{1}$, podemos corrigir $\widehat{\boldsymbol{\beta}}_{\mathrm{MQ}}$ tomando

$$
\widehat{\boldsymbol{\beta}}_{c}=\left(\mathbf{S}_{X X}-\widehat{\Sigma_{1}}\right)^{-1} s_{X Y},
$$

de forma que $\widehat{\boldsymbol{\beta}}_{c} \stackrel{\mathrm{qc}}{\longrightarrow} \boldsymbol{\beta}$. Seguindo Hwang (1986), assumimos que $\boldsymbol{\Sigma}_{u u}$ e $\boldsymbol{\Sigma}_{v v}$ são conhecidas. Pela lei forte dos grandes números segue que

$$
\mathbf{M}_{W W}=\frac{1}{k n} \sum_{i=1}^{k} \sum_{j=1}^{n} \mathbf{W}_{i j} \mathbf{W}_{i j}^{\prime} \stackrel{\mathrm{qc}}{\longrightarrow} \mathrm{E}\left(\mathbf{W}_{11} \mathbf{W}_{11}^{\prime}\right)=\left(\Sigma_{u u}+\mathbf{1}_{l} \mathbf{1}_{l}^{\prime}\right) \odot\left(\Sigma_{w w}+\boldsymbol{\mu}_{w} \boldsymbol{\mu}_{w}^{\prime}\right),
$$

e então,

$$
\widehat{\Sigma_{1}}=\left(\begin{array}{cc}
\mathrm{M}_{W W} \odot \Sigma_{u u} \oslash\left(\Sigma_{u u}+1_{l} 1_{l}^{\prime}\right) & 0 \\
& \Sigma_{v v}
\end{array}\right) \stackrel{\mathrm{qc}}{\longrightarrow} \Sigma_{1},
$$


como pretendido, onde " $\oslash$ " denota divisão elemento a elemento.

Incorporando a suposição abaixo, pertinente aos momentos de quarta ordem de $\mathrm{X}_{11}$, podemos estudar as distribuições assintóticas de $\widehat{\boldsymbol{\beta}}_{\mathrm{MQ}}$ e $\widehat{\boldsymbol{\beta}}_{c}$.

$\mathrm{S} 2.2$

$$
\mathrm{E}\left(\left\|\left(\boldsymbol{x}_{11}^{\prime}, \boldsymbol{u}_{11}^{\prime}, \boldsymbol{v}_{11}^{\prime}\right)^{\prime}\right\|^{4}\right)<\infty
$$

Lema 2.1 Sob o modelo (2.1)-(2.3), supondo válidas S2.1 e S2.2, temos

$$
\sqrt{n}\left(\widehat{\boldsymbol{\beta}}_{\mathrm{MQ}}-\Sigma_{X X}^{-1} \boldsymbol{\Sigma}_{x x} \boldsymbol{\beta}\right)=O_{p}(\mathbf{1}) \quad \text { e } \quad \sqrt{n}\left(\widehat{\boldsymbol{\beta}}_{c}-\boldsymbol{\beta}\right)=O_{p}(\mathbf{1}) \text {. }
$$

Prova. (Esboço) Consiste em justificar a existência das distribuições assintóticas (normais) de $\widehat{\boldsymbol{\beta}}_{\mathrm{MQ}}$ e $\widehat{\boldsymbol{\beta}}_{c}$. Recorrendo a (2.1) e S2.1, calculamos $\mathrm{E}\left(Y_{i j}\right)=\mu_{Y_{i}}=$ $\alpha+\tau_{i}+\boldsymbol{\mu}_{x}^{\prime} \boldsymbol{\beta}$. Devido às suposições $\mathrm{S} 2.1$ e S2.2, os vetores

$$
\left(\begin{array}{c}
\operatorname{vec}\left(\left(\mathbf{X}_{i j}-\boldsymbol{\mu}_{x}\right)\left(\mathbf{X}_{i j}-\boldsymbol{\mu}_{x}\right)^{\prime}\right) \\
\left(\mathbf{X}_{i j}-\boldsymbol{\mu}_{x}\right)\left(Y_{i j}-\mu_{Y_{i}}\right) \\
\operatorname{vec}\left(\mathbf{W}_{i j} \mathbf{W}_{i j}^{\prime}\right)
\end{array}\right), j=1, \ldots, n
$$

compõem uma amostra aleatória de uma distribuição com média

$$
\left(\begin{array}{c}
\operatorname{vec}\left(\boldsymbol{\Sigma}_{x x}+\boldsymbol{\Sigma}_{1}\right) \\
\boldsymbol{\Sigma}_{x x} \boldsymbol{\beta} \\
\operatorname{vec}\left(\mathrm{E}\left(\mathbf{W}_{11} \mathbf{W}_{11}^{\prime}\right)\right)
\end{array}\right)
$$

e matriz de covariâncias finita (singular), $i=1, \ldots, k$. O teorema central do limite, a lei forte dos grandes números e o teorema de Slutsky permitem encontrar a distribuição assintótica dos elementos presentes em (2.12) e (2.13). Em seguida o método delta (Sen \& Singer, 1993, Teorema 3.4.5) conduz ao resultado.

Propomos ainda um terceiro estimador de $\beta$, uma modificação de $\widehat{\boldsymbol{\beta}}_{c}$ baseada em Fuller (1987). Seja

$$
\widehat{\boldsymbol{\beta}}_{c}^{*}={\widehat{\Sigma_{x x}}}^{-1} s_{X Y},
$$

onde

$$
\widehat{\Sigma_{x x}}= \begin{cases}\mathbf{S}_{X X}-\widehat{\Sigma_{1}}, & \text { se } \widehat{f} \geq 1+\frac{1}{k n} \\ \mathbf{S}_{X X}-\left(\widehat{f}-\frac{1}{k n}\right) \widehat{\Sigma_{1}}, & \text { se } \widehat{f}<1+\frac{1}{k n}\end{cases}
$$


e $\widehat{f}$ é a menor raiz da equação

$$
\left|\left(\begin{array}{cc}
s_{Y Y} & s_{X Y}^{\prime} \\
& \mathrm{S}_{X X}
\end{array}\right)-f\left(\begin{array}{cc}
0 & 0 \\
& \widehat{\Sigma_{1}}
\end{array}\right)\right|=0,
$$

$|\mathrm{M}|$ é o determinante da matriz $\mathrm{M}, s_{Y Y}=\frac{1}{k(n-1)} \sum_{i=1}^{k} \sum_{j=1}^{n}\left(Y_{i j}-\bar{Y}_{i .}\right)^{2}, \mathrm{~S}_{X X}$ e $s_{X Y}$ são dadas em (2.11). As matrizes $\widehat{\Sigma_{x x}}$ e $\mathbf{S}_{X X}-\widehat{\Sigma_{1}}$ são estimadores consistentes $(\stackrel{\mathrm{qc}}{\longrightarrow})$ de $\Sigma_{x x}$, sendo que $\widehat{\Sigma_{x x}}$ é definida positiva, ao passo que $\mathrm{S}_{X X}-\widehat{\Sigma_{1}}$ pode não ser. Os estimadores $\widehat{\boldsymbol{\beta}}_{c}$ e $\widehat{\boldsymbol{\beta}}_{c}^{*}$ são assintoticamente equivalentes.

Substituindo as expressões (2.12), (2.13) e (2.14) em (2.5) obtemos três estimadores consistentes de $\tau$, designados por $\widehat{\tau}_{\mathrm{MQ}}, \widehat{\tau}_{c}$ e $\widehat{\tau}_{c}^{*}$, respectivamente. Concluímos do Teorema 2.1 que

$$
\begin{aligned}
& \sqrt{n}\left(\widehat{\tau}_{\mathrm{MQ}}-\tau\right) \stackrel{\mathrm{d}}{\longrightarrow} \mathrm{N}_{k}\left(0, \mathrm{C}_{k}\left(\sigma_{e e}+\beta^{\prime} \Sigma_{1} \Sigma_{X X}^{-1} \Sigma_{x x} \beta\right)\right) \mathrm{e} \\
& \sqrt{n}\left(\widehat{\tau}_{c}-\tau\right) \stackrel{\mathrm{d}}{\longrightarrow} \mathrm{N}_{k}\left(0, \mathrm{C}_{k}\left(\sigma_{e e}+\beta^{\prime} \Sigma_{1} \beta\right)\right),
\end{aligned}
$$

$\Sigma_{X X}$ e $\Sigma_{1}$ conforme (2.6) e (2.7). Tendo estas distribuições limite podemos comparar os estimadores.

Definição 2.1 Sejam $\widehat{\tau}_{i 1}$ e $\widehat{\tau}_{i 2}$ dois estimadores de $\tau_{i}$ tais que $\sqrt{n}\left(\widehat{\tau}_{i 1}-\tau_{i}\right) \stackrel{\mathrm{d}}{\longrightarrow}$ $\mathrm{N}\left(0, \sigma_{11}\right)$ e $\sqrt{n}\left(\widehat{\tau}_{i 2}-\tau_{i}\right) \stackrel{\mathrm{d}}{\longrightarrow} \mathrm{N}\left(0, \sigma_{22}\right)$. A eficiência relativa assintótica de $\widehat{\tau}_{i 1}$ em relação a $\widehat{\tau}_{i 2}$, denotada por $\epsilon\left(\widehat{\tau}_{i 1}, \widehat{\tau}_{i 2}\right)$, é definida por $\epsilon\left(\widehat{\tau}_{i 1}, \widehat{\tau}_{i 2}\right)=\sigma_{22} / \sigma_{11}$.

Com o auxílio de (2.15),

$$
\epsilon\left(\widehat{\tau}_{M Q_{i}}, \widehat{\tau}_{c_{i}}\right)=\frac{\sigma_{e e}+\beta^{\prime} \Sigma_{1} \beta}{\sigma_{e e}+\beta^{\prime} \Sigma_{1} \Sigma_{X X}^{-1} \Sigma_{x x} \beta} \quad, \quad i=1, \ldots, k .
$$

Portanto, $\epsilon\left(\widehat{\tau}_{\mathrm{MQ}_{i}}, \widehat{\tau}_{c_{\mathrm{i}}}\right) \geq 1$, pois $\Sigma_{1} \Sigma_{X X}^{-1} \Sigma_{x x}=\Sigma_{1} \Sigma_{X X}^{-1}\left(\Sigma_{X X}-\Sigma_{1}\right)=\Sigma_{1}-\Sigma_{1} \Sigma_{X X}^{-1} \Sigma_{1}$. O estimador $\widehat{\tau}_{\mathrm{MQ}}$, assintoticamente, é pelo menos tão eficiente quanto $\widehat{\tau}_{c}$.

Completamos apresentando estimadores para os demais parâmetros. Estimadores fortemente consistentes para $\alpha$ e $\boldsymbol{\mu}_{x}$ são dados por

$$
\widehat{\alpha}=\bar{Y}_{. .}-\overline{\mathbf{X}}_{. .}^{\prime} \widehat{\boldsymbol{\beta}}_{c}^{*} \text { e } \widehat{\mu_{x}}=\overline{\mathbf{X}} .
$$

$\operatorname{com} \bar{Y}_{. .}, \overline{\mathbf{X}}_{. .}$e $\overline{\mathbf{X}}$. vindas de (2.4). Sejam $\mathbf{Y}_{. j}=\left(Y_{1 j}, \ldots, Y_{k j}\right)^{\prime}, j=1, \ldots, n$, e $\mathrm{Y}=\left(\mathrm{Y}_{.1}^{\prime}, \ldots, \mathrm{Y}_{. n}^{\prime}\right)^{\prime} ; \mathrm{X}$ definido da mesma forma que $\mathbf{Y}$. Procedendo como Hwang (1986), um estimador de $\sigma_{e e}$ tem expressão

$$
\widehat{\sigma_{e e}}=\max \left\{0,{\widehat{\sigma_{e e}}}^{*}\right\},
$$


Tabela 2.1: Eficiências relativas assintóticas teóricas.

\begin{tabular}{cccccc}
\hline$\kappa_{w}=\kappa_{z}$ & 0.1 & 0.3 & 0.5 & 0.7 & 0.9 \\
$\epsilon\left(\widehat{\tau}_{\mathrm{MQ}_{1}}, \widehat{\tau}_{c_{1}}^{*}\right)$ & 2.50 & 1.88 & 1.50 & 1.25 & 1.07 \\
\hline
\end{tabular}

onde

$$
\begin{aligned}
{\widehat{\sigma_{e e}}}^{*}= & \frac{1}{k n}\left[\left\|\mathbf{Y}-\widehat{\alpha} \mathbf{1}_{n k}-\mathbf{1}_{n} \otimes \widehat{\tau}_{\mathrm{MQ}}\right\|^{2}-2\left(\mathbf{Y}-\widehat{\alpha} \mathbf{1}_{n k}-\mathbf{1}_{n} \otimes \widehat{\boldsymbol{\tau}}_{\mathrm{MQ}}\right)^{\prime} \mathbf{X}^{\prime} \widehat{\boldsymbol{\beta}}_{c}^{*}\right. \\
& \left.+\widehat{\boldsymbol{\beta}}_{c}^{* \prime}\left(\mathbf{X X}^{\prime}-n k \widehat{\Sigma_{1}}\right) \widehat{\boldsymbol{\beta}}_{c}^{*}\right] .
\end{aligned}
$$

\subsection{Simulações}

Esta seção traz resultados de um pequeno estudo de simulação comparando os méritos relativos dos estimadores propostos. Selecionamos o modelo mais simples, ou seja, um modelo com dois grupos $(k=2)$, duas covariáveis $(p=2, l=1)$ - uma delas medida com erro multiplicativo e a outra medida com erro aditivo -, e sem correlação entre as covariáveis. Sendo assim, a eficiência relativa assintótica é dada por

$$
\epsilon\left(\widehat{\tau}_{\mathrm{MQ}_{1}}, \widehat{\tau}_{c_{1}}\right)=\frac{1+\beta_{1}^{2} \delta_{u}+\beta_{2}^{2} \delta_{v}}{1+\kappa_{w} \beta_{1}^{2} \delta_{u}+\kappa_{z} \beta_{2}^{2} \delta_{v}}
$$

onde $\delta_{u}=\frac{\sigma_{u u}^{*}}{\sigma_{e e}}, \delta_{v}=\frac{\sigma_{v v}}{\sigma_{e e}}, \kappa_{w}=\frac{\sigma_{w w}}{\sigma_{w w}+\sigma_{u u}^{*}}, \kappa_{z}=\frac{\sigma_{z z}}{\sigma_{z z}+\sigma_{v v}}$ e $\sigma_{u u}^{*}=\sigma_{u u}\left(\sigma_{w w}+\mu_{w}^{2}\right)$. Selecionando $\beta_{1}=\beta_{2}=\delta_{u}=\delta_{v}=1$ obtemos uma expressão mais simples,

$$
\epsilon\left(\widehat{\tau}_{\mathrm{MQ}_{1}}, \widehat{\tau}_{c_{1}}\right)=\frac{3}{1+\kappa_{w}+\kappa_{z}}
$$

e a Tabela 2.1 contém valores de $\epsilon\left(\widehat{\tau}_{\mathrm{MQ}_{1}}, \widehat{\tau}_{c_{1}}\right)$ para alguns valores de $\kappa_{w}=\kappa_{z}$, refletindo em algum sentido diferentes quantidades de erro de medição. Quando $\sigma_{u u}$ ou (e) $\sigma_{v v}$ $\operatorname{aumenta}(\mathrm{m})$ (a quantidade de erro de medição aumenta), mais eficiente se torna o estimador de mínimos quadrados de $\tau_{1}$.

A Tabela 2.2 juntamente com a Tabela 2.1 completa a especificação dos parâmetros do modelo. Finalmente, selecionamos as seguintes distribuições para os componentes estocásticos:

$$
\begin{array}{ll}
e_{11} \sim \mathrm{N}\left(0, \sigma_{e e}\right), & u_{11} \sim \text { Uniforme }\left(1-\sqrt{3 \sigma_{u u}}, 1+\sqrt{3 \sigma_{u u}}\right), \\
v_{11} \sim \mathrm{N}\left(0, \sigma_{v v}\right), & w_{11} \sim \mathrm{N}\left(\mu_{\cdot w}, \sigma_{w w}\right) \text { e } z_{11} \sim \mathrm{N}\left(\mu_{z}, \sigma_{z z}\right) .
\end{array}
$$


Tabela 2.2: Valores dos parâmetros usados nas simulações.

\begin{tabular}{cccccc}
\hline$\alpha$ & $\tau_{1}$ & $\beta_{1}$ & $\beta_{2}$ & $\mu_{w}$ & $\mu_{z}$ \\
10 & 1 & 1 & 1 & 1 & 10 \\
\hline$\sigma_{e e}$ & $\sigma_{u u}$ & $\sigma_{v v}$ & $\sigma_{w w}$ & $\sigma_{w z}$ & $\sigma_{z z}$ \\
$\frac{1-\kappa_{z}}{\kappa_{z}}$ & $\frac{1-\kappa_{w}}{2 \kappa_{w}}$ & $\frac{1-\kappa_{z}}{\kappa_{z}}$ & 1 & 0 & 1 \\
\hline
\end{tabular}

As simulações foram realizadas em linguagem Ox (Doornik, 1998). Valores dos vícios simulados são apresentados na Tabela 2.3 para amostras de tamanho pequeno a moderado. Para cada combinação de tamanho amostral $(n)$ e $\kappa_{w}=\kappa_{z}, 1000$ amostras aleatórias foram geradas, e para cada amostra foram calculados $\widehat{\tau}_{\mathrm{MQ}_{1}}, \widehat{\tau}_{c_{1}}$ e $\widehat{\tau}_{c_{1}}^{*}$. O vício simulado do estimador $\widehat{\tau}_{M Q_{1}}$, por exemplo, é $\sum_{s=1}^{1000}\left(\widehat{\tau}_{M_{1, s}}-\tau_{1}\right) / 1000$. As três linhas em cada bloco da tabela correspondem aos vícios de $\widehat{\tau}_{\mathrm{MQ}_{1}}, \widehat{\tau}_{c_{1}}$ e $\widehat{\tau}_{c_{1}}^{*}$, respectivamente. Na maioria das situações da Tabela 2.3 o estimador de mínimos quadrados apresenta vícios simulados mais próximos de 0 . Notamos maiores diferenças entre os três estimadores na parte acima da linha divisória, referente às combinações mais extremas em termos de erros de medição e (ou) tamanho amostral. O estimador modificado $\widehat{\tau}_{c_{1}}^{*}$ nitidamente é um aperfeiçoamento comparado a $\widehat{\tau}_{c_{1}}$.

Eficiências relativas simuladas encontram-se na Tabela 2.4. O erro quadrático médio simulado (EQMS) do estimador $\widehat{\tau}_{\mathrm{MQ}_{1}}$ é dado por $\sum_{s=1}^{1000}\left(\widehat{\tau}_{\mathrm{MQ}_{1, s}}-\tau_{1}\right)^{2} / 1000$. Definimos eficiência relativa simulada entre dois estimadores como sendo a razão entre seus EQMS. Tomando $\widehat{\tau}_{M Q_{1}}$ e $\widehat{\tau}_{c_{1}}$,

$$
\epsilon\left(\widehat{\tau}_{\mathrm{MQ}_{1}}, \widehat{\tau}_{c_{1}}\right) \text { simulada }=\frac{\operatorname{EQMS}\left(\widehat{\tau}_{c_{1}}\right)}{\operatorname{EQMS}\left(\widehat{\tau}_{\mathrm{MQ}_{1}}\right)} .
$$

Os valores em cada bloco da Tabela 2.4 representam as eficiências relativas simuladas de $\widehat{\tau}_{\mathrm{MQ}_{1}}$ em relação a $\widehat{\tau}_{c_{1}}$ e $\widehat{\tau}_{c_{1}}^{*}$, nesta ordem. Assim como na Tabela 2.3, a Tabela 2.4 pode ser dividida em duas partes. Na região acima da linha as eficiências simuladas têm valores bastante diferentes dos valores teóricos (Tabela 2.1), principalmente se confrontarmos $\widehat{\tau}_{\mathrm{MQ}_{1}}$ e $\widehat{\tau}_{c_{1}}$, podendo ser conseqüência de maiores (em valores absolutos) vícios simulados. Novamente, $\widehat{\tau}_{c_{1}}^{*}$ é um avanço em relação a $\widehat{\tau}_{c_{1}}$. Nos blocos abaixo da divisória (condições menos extremas) os três estimadores têm comportamento semelhante nas duas tabelas. 
Tabela 2.3: Vícios simulados dos estimadores $\widehat{\tau}_{M_{Q_{1}}}, \widehat{\tau}_{c_{1}}$ e $\widehat{\tau}_{c_{1}}^{*}$.

\begin{tabular}{|c|c|c|c|}
\hline \multirow[b]{2}{*}{$\kappa_{w}=\kappa_{z}$} & \multicolumn{3}{|c|}{ Tamanho da amostra $(n)$} \\
\hline & 10 & 50 & 100 \\
\hline \multirow[t]{3}{*}{0.1} & 0.02160 & -0.00261 & 0.00997 \\
\hline & 0.10464 & -1.32175 & -0.31567 \\
\hline & 0.00349 & 0.01873 & -0.00001 \\
\hline \multirow[t]{3}{*}{0.3} & -0.00737 & -0.00766 & -0.00161 \\
\hline & 0.08428 & -0.05318 & -0.00725 \\
\hline & 0.00709 & -0.01537 & -0.00487 \\
\hline \multirow[t]{3}{*}{0.5} & 0.00773 & 0.00606 & 0.00370 \\
\hline & -0.06705 & 0.00656 & 0.00474 \\
\hline & 0.00377 & 0.00669 & 0.00473 \\
\hline \multirow[t]{3}{*}{0.7} & -0.00272 & -0.00730 & 0.00036 \\
\hline & -0.01927 & -0.00708 & 0.00116 \\
\hline & -0.00769 & -0.00713 & 0.00116 \\
\hline \multirow[t]{3}{*}{0.9} & -0.00528 & -0.00053 & 0.00112 \\
\hline & -0.00798 & -0.00008 & 0.00145 \\
\hline & -0.00723 & -0.00009 & 0.00145 \\
\hline
\end{tabular}


Tabela 2.4: Eficiências relativas simuladas de $\widehat{\tau}_{M_{Q_{1}}}$ em relação a $\widehat{\tau}_{c_{1}}$ e $\widehat{\tau}_{c_{1}}^{*}$.

\begin{tabular}{|c|c|c|c|}
\hline \multirow[b]{2}{*}{$\kappa_{w}=\kappa_{z}$} & \multicolumn{3}{|c|}{ Tamamnho da amostra $(n)$} \\
\hline & 10 & 50 & 100 \\
\hline \multirow[t]{2}{*}{0.1} & 1076. & 3779. & 928.4 \\
\hline & 2.766 & 7.999 & 7.615 \\
\hline \multirow[t]{2}{*}{0.3} & 82.03 & 96.44 & 2.641 \\
\hline & 2.508 & 3.303 & 2.242 \\
\hline \multirow[t]{2}{*}{0.5} & 35.55 & 1.809 & 1.570 \\
\hline & 2.342 & 1.781 & 1.570 \\
\hline \multirow[t]{2}{*}{0.7} & 11.23 & 1.306 & 1.309 \\
\hline & 1.605 & 1.299 & 1.309 \\
\hline \multirow[t]{2}{*}{0.9} & 1.174 & 1.100 & 1.045 \\
\hline & 1.139 & 1.100 & 1.045 \\
\hline
\end{tabular}

Em certas situações existem amostras em que a matriz $\mathbf{S}_{X X}-\widehat{\boldsymbol{\Sigma}_{1}}$ não é definida positiva. Por exemplo, com $\kappa_{w}=\kappa_{z}=0.1$ e $n=10$ contamos 3532 de tais amostras, que foram excluídas da simulação.

Chen (1997) propõe um estimador para os efeitos dos tratamentos $(\boldsymbol{\tau})$ a partir de uma equação de estimação baseada em postos. Estudos de simulação parecem indicar a superioridade do estimador proposto em relação ao estimador de mínimos quadrados $\left(\widehat{\tau}_{\mathrm{MQ}}\right)$. Entretanto, não é possível efetuarmos comparações diretas entre os estimadores através de expressões do tipo (2.16), pois não se dispõe da distribuição assintótica do estimador proposto por Chen (1997).

\subsection{Extensões}

Estimadores consistentes de $\beta$ foram construídos supondo conhecidas as matrizes de covariâncias dos erros de medição nas covariáveis $\left(\Sigma_{u u}\right.$ e $\left.\Sigma_{v v}\right)$. Se tivermos um estimador consistente da matriz $\Psi=\Sigma_{X X}^{-1} \Sigma_{x x}(\widehat{\Psi}$, digamos $)$, o estimador $\widehat{\Psi}^{-1} \widehat{\boldsymbol{\beta}}_{\mathrm{MQ}}$ também é consistente para $\boldsymbol{\beta}$. Gleser $(1992,1993)$ chama $\boldsymbol{\Psi}$ de matriz de confiabilidade das medições $\mathrm{X}_{i j}$ e ressalta que informação sobre $\Psi$ pode ser obtida de estudos de confiabilidade. 
Modelos mais gerais que o modelo ANCOVA poderiam ter sido formulados. Seria de interesse pesquisar combinações lineares do vetor de parâmetros do modelo cujos estimadores de mínimos quadrados são consistentes e mais eficientes assintoticamente que outros estimadores. Resultados preliminares em um modelo linear com três tipos de preditores - medidos sem erros, medidos com erros aditivos e medidos com erros multiplicativos - indicam que, se providenciarmos algumas suposições, é possível estender os resultados de Gleser, Carroll \& Gallo (1987), que estudam um modelo com os dois primeiros tipos de preditores acima. 


\section{Capítulo 3}

\section{Influência Local no Modelo de Calibração Comparativa}

\subsection{Introdução}

Neste capítulo estudamos o problema de calibração comparativa, no qual uma. mesma quantidade desconhecida é medida por instrumentos (ou métodos) diferentes. Dispomos de $p+1$ instrumentos ( $p \geq 1)$ para medir uma característica (ou resposta) $x$ em um grupo de $n$ unidades experimentais. Os modelos de calibração comparativa são casos particulares dos modelos com erros nas variáveis multivariados (Fuller, 1987). Alguns exemplos de aplicação nas áreas industrial e médica, Psicologia e Educação são fornecidos por Grubbs (1948, 1973), Barnett (1969), Carter (1981), Kelly (1984, 1985), Jaech (1985), Dunn (1989) e Chipkevitch, Nishimura, Tu \& Galea-Rojas (1996).

Sejam $x_{i}$ o verdadeiro valor da característica na unidade $i$ e $y_{i j}$ a medição dessa característica fornecida usando o instrumento $j, i=1, \ldots, n$ e $j=0,1, \ldots, p$. Da. mesma forma que Barnett (1969) e Kimura (1992), assumimos que $y_{i j}$ e $x_{i}$ obedecem à relação linear

$$
y_{i j}=\alpha_{j}+\beta_{j} x_{i}+\epsilon_{i j}
$$

$\operatorname{com} \epsilon_{i j} \stackrel{\text { indep. }}{\sim} \mathrm{N}\left(0, \sigma_{\epsilon \epsilon}\right), i=1, \ldots, n$ e $j=0,1, \ldots, p$. Os parâmetros $\alpha_{j}$ e $\beta_{j}$ representam os vícios aditivo e multiplicativo, respectivamente, do instrumento $j$. Fazendo

$$
\mathbf{Y}_{i}=\left(y_{i 0}, y_{i 1}, \ldots, y_{i p}\right)^{\prime}, \quad \epsilon_{i}=\left(\epsilon_{i 0}, \epsilon_{i 1}, \ldots, \epsilon_{i p}\right)^{\prime}
$$


- vetores aleatórios de dimensão $r=p+1-, \alpha=\left(\alpha_{1}, \ldots, \alpha_{p}\right)^{\prime}$ e $\beta=\left(\beta_{1}, \ldots, \beta_{p}\right)^{\prime}$, matricialmente o modelo tem expressão

$$
\mathbf{Y}_{i}=\alpha+\beta x_{i}+\epsilon_{i}
$$

$i=1, \ldots, n$, denominada parametrização de regressão dos modelos de calibração comparativa. Supomos ainda que

$$
\epsilon_{i} \stackrel{\text { iid }}{\sim} \mathrm{N}_{r}\left(0, \Sigma_{\epsilon \epsilon}\right)
$$

$i=1, \ldots, n$. Em relação à matriz $\Sigma_{\epsilon \epsilon}$ trataremos duas situações distintas:

1. $\Sigma_{\epsilon \epsilon}=\sigma_{\epsilon \epsilon} \Sigma_{0}, \Sigma_{0}$ conhecida e sem perda de generalidade tomamos $\Sigma_{0}=\mathbf{I}_{r}$ (Modelo homoscedástico - Seção 3.2) e

2. $\Sigma_{\epsilon \epsilon}=\mathbf{D}\left(\sigma_{\epsilon \epsilon}\right)$, onde $\mathbf{D}\left(\epsilon_{\epsilon \epsilon}\right)=\operatorname{diag}\left(\sigma_{\epsilon \epsilon 0}, \sigma_{\epsilon \epsilon 1}, \ldots, \sigma_{\epsilon \epsilon p}\right)$ é a matriz diagonal formada pelos elementos do vetor $\boldsymbol{\sigma}_{\epsilon \epsilon}=\left(\sigma_{\epsilon \epsilon 0}, \sigma_{\epsilon \epsilon 1}, \ldots, \sigma_{\epsilon \epsilon p}\right)^{\prime}$ (Modelo heteroscedástico - Seção 3.3).

Ressaltamos que se $p=1$ temos o caso particular do modelo de regressão linear simples com erros nas variáveis (Fuller, 1987; Cheng \& Van Ness, 1999).

Em relação à quantidade desconhecida $(x)$ consideraremos dois tipos de modelo:

1. Funcional: $x_{1}, \ldots, x_{n}$ é uma seqüência de reais, denominados parâmetros incidentais. Neste caso,

$$
\mathbf{Y}_{i} \stackrel{\text { indep. }}{\sim} \mathrm{N}_{r}\left(\boldsymbol{\alpha}+\boldsymbol{\beta} x_{i}, \boldsymbol{\Sigma}_{\epsilon \epsilon}\right), i=1, \ldots, n
$$

2. Estrutural: $x_{1}, \ldots, x_{n}$ são variáveis aleatórias hipoteticamente $\stackrel{\mathrm{iid}}{\sim} \mathrm{N}\left(\mu_{x}, \sigma_{x x}\right)$ e independentes de $\epsilon_{i}, i=1, \ldots, n$, de modo que

$$
\mathbf{Y}_{1}, \ldots, \mathbf{Y}_{n} \stackrel{\text { iid }}{\sim} \mathrm{N}_{r}\left(\alpha+\beta \mu_{x}, \mathrm{~V}\right)
$$

onde $\mathrm{V}=\sigma_{x x} \beta \beta^{\prime}+\Sigma_{\epsilon \epsilon}$.

Combinando as suposições sobre a matriz $\Sigma_{\epsilon \epsilon}$ e a seqüência $x_{1}, \ldots, x_{n}$ totalizamos quatro tipos diferentes de modelo. Os modelos estruturais são inidentificáveis (fato provado em Galea-Rojas, 1995). A falta de identificabilidade pode ser contornada impondo restrições sobre os parâmetros do modelo. Barnett (1969) assume 
a existência de um instrumento de referência (instrumento 0) efetuando medições sem vícios $\left(\alpha_{0}=0\right.$ e $\left.\beta_{0}=1\right)$. Este cenário é justificável em diversas situações práticas em que um instrumento mais sofisticado (um procedimento bem conhecido, um método bem desenvolvido, ... ) serve como padrão. Esta restrição será adotada neste capítulo, permitindo obter estimadores de máxima verossimilhança (EMV), para $p \geq 2$, nos modelos homoscedásticos $\left(\Sigma_{\epsilon \epsilon}=\sigma_{\epsilon \epsilon} \mathbf{I}_{r}\right)$ e no modelo estrutural heteroscedástico $\left(\Sigma_{\epsilon \epsilon}=\mathbf{D}\left(\boldsymbol{\sigma}_{\epsilon \epsilon}\right)\right)$, conforme será visto nas seções 3.2 e 3.3. O função verossimilhança do modelo funcional heteroscedástico é ilimitado, de acordo com Gleser (1981, Teorema 5.1). Em (3.1) as relações passam a ser

$$
\begin{gathered}
y_{i 0}=x_{i}+\epsilon_{i 0}, \\
y_{i j}=\alpha_{j}+\beta_{j} x_{i}+\epsilon_{i j},
\end{gathered}
$$

$i=1, \ldots, n$ e $j=1, \ldots, p$. Para $\beta_{j} \neq 0$, definimos uma medição calibrada realizada com o instrumento $j$ na unidade $i$ como sendo

$$
\frac{y_{i j}-\alpha_{j}}{\beta_{j}}
$$

$i=1, \ldots, n$ e $j=1, \ldots, p$. Na prática utilizamos estimativas com $\widehat{\alpha}$ e $\widehat{\beta}$ no lugar de $\alpha$ e $\beta$.

Redefinimos $\alpha$ e $\beta$ como

$$
\boldsymbol{\alpha}=\left(\alpha_{1}, \ldots, \alpha_{p}\right)^{\prime} \text { e } \boldsymbol{\beta}=\left(\beta_{1}, \ldots, \beta_{p}\right)^{\prime},
$$

levando a uma reformulação dos modelos:

$$
\mathbf{Y}_{i}=\left(\begin{array}{c}
0 \\
\alpha
\end{array}\right)+\left(\begin{array}{l}
1 \\
\beta
\end{array}\right) x_{i}+\epsilon_{i}
$$

$i=1, \ldots, n \mathrm{e}$

1. Funcional:

$$
\mathbf{Y}_{i} \stackrel{\text { indep. }}{\sim} \mathrm{N}_{r}\left(\left(\begin{array}{c}
0 \\
\alpha
\end{array}\right)+\left(\begin{array}{c}
1 \\
\beta
\end{array}\right) x_{i}, \Sigma_{\epsilon \epsilon}\right), \quad i=1, \ldots, n
$$

2. Estrutural:

$$
\mathrm{Y}_{1}, \ldots, \mathrm{Y}_{n} \stackrel{\text { iid }}{\sim} \mathrm{N}_{r}\left(\left(\begin{array}{c}
0 \\
\alpha
\end{array}\right)+\left(\begin{array}{l}
1 \\
\beta
\end{array}\right) \mu_{x}, \mathrm{~V}\right)
$$


onde

$$
\mathrm{V}=\sigma_{x x}\left(\begin{array}{l}
1 \\
\beta
\end{array}\right)\left(\begin{array}{l}
1 \\
\beta
\end{array}\right)^{\prime}+\Sigma_{\epsilon \epsilon}
$$

Modelos estatísticos quase sempre constituem representações simplificadas de processos complexos. Sendo assim, parte importante de uma análise estatística é o estudo das possíveis variações nos resultados ocasionadas por pequenas modificações na formulação do problema. Na Seção 3.5 analisaremos as variações causadas por diferentes perturbações do modelo proposto sobre os EMV dos parâmetros do modelo. Para tanto, recorremos à técnica de avaliação de influência local proposta em Cook (1986), resumida na Seção 3.4. Por fim, na Seção 3.6 a técnica é aplicada a conjuntos de dados reais.

\subsection{O modelo homoscedástico}

Em (3.4) e (3.5) a matriz de covariâncias dos erros de medição é $\Sigma_{\epsilon \epsilon}=\sigma_{\epsilon \epsilon} \mathbf{I}_{r}$. Expressamos os logaritmos das funções verossimilhança na forma

$$
\mathcal{L}(\theta ; \mathbf{Y})=\sum_{i=1}^{n} l\left(\theta ; \mathbf{Y}_{i}\right)
$$

onde $\mathrm{Y}=\left(\mathrm{Y}_{1}^{\prime}, \ldots, \mathrm{Y}_{n}^{\prime}\right)$.

No modelo funcional, $\theta=\left(\boldsymbol{\alpha}^{\prime}, \boldsymbol{\beta}^{\prime}, \sigma_{\epsilon \epsilon}, x_{1}, \ldots, x_{n}\right)^{\prime} \mathrm{e}$

$$
l\left(\boldsymbol{\theta} ; \mathbf{Y}_{i}\right)=-\frac{r}{2} \log 2 \pi-\frac{r}{2} \log \sigma_{\epsilon \epsilon}-\frac{1}{2} Q_{F}\left(\boldsymbol{\theta} ; \mathbf{Y}_{i}\right)
$$

onde

$$
Q_{F}\left(\boldsymbol{\theta} ; \mathbf{Y}_{i}\right)=\frac{1}{\sigma_{\epsilon \epsilon}}\left\{\left(y_{i 0}-x_{i}\right)^{2}+\left\|\mathbf{Y}_{1 i}-\left(\alpha+\beta x_{i}\right)\right\|^{2}\right\}
$$

$\operatorname{com} \mathbf{Y}_{1 i}=\left(y_{i 1}, \ldots, y_{i p}\right)^{\prime}, i=1, \ldots, n$.

No modelo estrutural, $\boldsymbol{\theta}=\left(\boldsymbol{\alpha}^{\prime}, \boldsymbol{\beta}^{\prime}, \sigma_{\epsilon \epsilon}, \mu_{x}, \sigma_{x x}\right)^{\prime}$ e fazendo

$$
\mathbf{b}=\left(1, \boldsymbol{\beta}^{\prime}\right)^{\prime}, c=\mathbf{b}^{\prime} \mathbf{b} \quad \text { e } \quad h=\frac{\sigma_{x x}}{\sigma_{\epsilon \epsilon} c}
$$

segue que

$$
\mathbf{V}^{-1}=\frac{1}{\sigma_{\epsilon \epsilon}}\left(\mathbf{I}_{r}-h \mathbf{b b}^{\prime}\right) \quad \text { e }|\mathrm{V}|=\frac{\sigma_{\epsilon \epsilon} \sigma_{x x}}{h}
$$


de modo que

$$
\begin{gathered}
l\left(\boldsymbol{\theta} ; \mathbf{Y}_{i}\right)=-\frac{r}{2} \log 2 \pi-\frac{1}{2} \log \frac{\sigma_{\epsilon \epsilon} \sigma_{x x}}{h}-\frac{1}{2} Q_{E}\left(\boldsymbol{\theta} ; \mathbf{Y}_{i}\right) \\
Q_{E}\left(\boldsymbol{\theta} ; \mathbf{Y}_{i}\right)=\left(\mathbf{Y}_{i}-\mathrm{E}\left(\mathbf{Y}_{i}\right)\right)^{\prime} \mathbf{V}^{-1}\left(\mathbf{Y}_{i}-\mathrm{E}\left(\mathbf{Y}_{i}\right)\right)=\frac{1}{\sigma_{\epsilon \epsilon}}\left(q_{1 i}-h q_{2 i}^{2}\right)
\end{gathered}
$$

$\mathrm{com}$

$$
\begin{aligned}
& q_{1 i}=\left(y_{i 0}-\mu_{x}\right)^{2}+\left(\mathbf{Y}_{1 i}-\boldsymbol{\alpha}-\boldsymbol{\beta} \mu_{x}\right)^{\prime}\left(\mathbf{Y}_{1 i}-\boldsymbol{\alpha}-\boldsymbol{\beta} \mu_{x}\right) \quad \mathrm{e} \\
& q_{2 i}=\left(y_{i 0}-\mu_{x}\right)+\left(\mathbf{Y}_{1 i}-\boldsymbol{\alpha}-\boldsymbol{\beta} \mu_{x}\right)^{\prime} \boldsymbol{\beta},
\end{aligned}
$$

$i=1, \ldots, n$.

Em seguida apresentamos expressões dos EMV dos parâmetros e das matrizes de informação observadas, utilizadas no estudo de influência da Seção 3.5.

\subsubsection{EMV}

De acordo com Fuller (1987, p. 294), dados $\boldsymbol{\alpha}$ e $\boldsymbol{\beta}$ os EMV dos parâmetros incidentais $x_{i}$ no modelo funcional são calculados por

$$
\widehat{x_{i}}=\widehat{x_{i}}(\boldsymbol{\alpha}, \boldsymbol{\beta})=\frac{1}{c}\left[y_{i 0}+\left(\mathbf{Y}_{1 i}-\boldsymbol{\alpha}\right)^{\prime} \boldsymbol{\beta}\right],
$$

$i=1, \ldots, n$, onde $c$ e $\mathbf{Y}_{1 i}$ estão definidos em (3.9) e (3.8), respectivamente. Substituindo $\widehat{x_{i}}$ em (3.8) resulta em

$$
Q_{F}\left(\boldsymbol{\theta} ; \mathbf{Y}_{i}\right)=\frac{1}{\sigma_{\epsilon \epsilon}}\left\{y_{i 0}^{2}+\left\|\mathbf{Y}_{1 i}-\boldsymbol{\alpha}\right\|^{2}-\frac{1}{c}\left[y_{i 0}+\left(\mathbf{Y}_{1 i}-\boldsymbol{\alpha}\right)^{\prime} \boldsymbol{\beta}\right]^{2}\right\}
$$

$i=1, \ldots, n$, onde $\theta=\left(\boldsymbol{\alpha}^{\prime}, \boldsymbol{\beta}^{\prime}, \sigma_{\epsilon \epsilon}\right)^{\prime}$ é, de agora em diante, o vetor de parâmetros de maior relevância, para o qual é possível apontar um estimador consistente. Substituindo $Q_{F}$ acima em (3.7) conduz ao logaritmo da função verossimilhança perfilada em (3.6).

As expressões dos EMV vêm de Gleser (1981) e Shyr (1984). Sejam $d_{1} \geq d_{2} \geq$ $\ldots \geq d_{r}(r=p+1)$ os autovalores da matriz dos segundos momentos centrais amostrais

$$
\mathbf{S}=\frac{1}{n} \sum_{i=1}^{n}\left(\mathbf{Y}_{i}-\overline{\mathbf{Y}}\right)\left(\mathbf{Y}_{i}-\overline{\mathbf{Y}}\right)^{\prime}
$$

$\operatorname{com} \overline{\mathrm{Y}}=\sum_{i=1}^{n} \mathrm{Y}_{i} / n$, e cuja decomposição espectral é

$$
\mathrm{S}=\mathrm{GD}(d) \mathrm{G}^{\prime},
$$


onde $\mathbf{G}$ é uma matriz ortogonal $r \times r$ tendo na $j$-ésima coluna o autovetor associado a $d_{j}, j=0,1, \ldots, p$. Escrevendo

$$
\mathbf{G}=\left(\begin{array}{cc}
g_{11} & \mathrm{~g}_{12} \\
(1 \times 1) & (1 \times p) \\
\mathrm{g}_{21} & \mathbf{G}_{22} \\
(p \times 1) & (p \times p)
\end{array}\right)
$$

os EMV são dados por

$$
\begin{gathered}
\widehat{\alpha}=\overline{\mathrm{Y}}_{1}-\overline{y_{0}} \widehat{\boldsymbol{\beta}} \\
\widehat{\boldsymbol{\beta}}=\frac{g_{21}}{g_{11}} \text { e } \widehat{\sigma_{\epsilon \epsilon}}=\frac{1}{r} \sum_{i=2}^{r} d_{i}
\end{gathered}
$$

sendo que

$$
\overline{\mathbf{Y}}_{1}=\frac{1}{n} \sum_{i=1}^{n} \mathbf{Y}_{1 i} \quad \text { e } \quad \overline{y_{0}}=\frac{1}{n} \sum_{i=1}^{n} y_{i 0}
$$

e no modelo estrutural,

$$
\widehat{\sigma_{\epsilon \epsilon}}=\frac{1}{p} \sum_{i=2}^{r} d_{i}, \quad \widehat{\mu_{x}}=\overline{y_{0}} \quad \text { e } \widehat{\sigma_{x x}}=\frac{p d_{1}-\sum_{i=2}^{r} d_{i}}{p \widehat{c}}
$$

$\operatorname{com} \widehat{c}=1+\widehat{\boldsymbol{\beta}}^{\prime} \widehat{\boldsymbol{\beta}}$

\subsubsection{Matriz de informação observada}

Para o modelo funcional o logaritmo da função verossimilhança segue de (3.6), (3.7) e (3.14). A matriz de derivadas de segunda ordem de $\mathcal{L}(\boldsymbol{\theta} ; \mathbf{Y})$ em relação a $\theta=\left(\alpha^{\prime}, \beta^{\prime}, \sigma_{\epsilon \epsilon}\right)^{\prime}$ é representada em blocos por

$$
\ddot{\mathbf{L}}_{F}=\left.\frac{\partial^{2} \mathcal{L}(\boldsymbol{\theta} ; \mathbf{Y})}{\partial \theta \partial \boldsymbol{\theta}^{\prime}}\right|_{\boldsymbol{\theta}=\hat{\boldsymbol{\theta}}}=\left(\begin{array}{ccc}
\mathbf{L}_{\alpha \alpha} & \mathbf{L}_{\alpha \beta} & \mathbf{L}_{\alpha \sigma_{\epsilon \epsilon}} \\
& \mathbf{L}_{\beta \beta} & \mathbf{L}_{\beta \sigma_{\epsilon \epsilon}} \\
& & \mathbf{L}_{\sigma_{\epsilon \epsilon} \sigma_{\epsilon \epsilon}}
\end{array}\right)_{\left.\right|_{\boldsymbol{\theta}=\hat{\theta}}},
$$

onde $\widehat{\theta}$ é o EMV de $\theta$ (Seção 3.2.1) e, após várias manipulações algébricas,

$$
\mathbf{L}_{\alpha \alpha}=-\frac{n}{\sigma_{\epsilon \epsilon}}\left(\mathbf{I}_{p}-\frac{\beta \beta^{\prime}}{c}\right), \mathbf{L}_{\alpha \beta}=\overline{y_{0}} \mathbf{L}_{\alpha \alpha}, \quad \mathbf{L}_{\alpha \sigma_{\epsilon \epsilon}}=0
$$




$$
\begin{gathered}
\mathbf{L}_{\beta \beta}=\frac{n}{c \sigma_{\epsilon \epsilon}}\left[\mathbf{S}_{11}-c{\overline{y_{0}}}^{2}\left(\mathbf{I}_{p}-\frac{\beta \beta^{\prime}}{c}\right)-\frac{2 \mathbf{S}^{*}}{c}-\frac{\boldsymbol{b}^{\prime} \mathbf{S} b}{c}\left(\mathbf{I}_{p}-\frac{4 \beta \beta^{\prime}}{c}\right)\right] \\
\mathbf{L}_{\beta \sigma_{\epsilon \epsilon}}=0 \quad \text { e } \quad \mathbf{L}_{\sigma_{\epsilon \epsilon} \sigma_{\epsilon \epsilon}}=\frac{n r}{2 \sigma_{\epsilon \epsilon}^{2}}-\frac{1}{\sigma_{\epsilon \epsilon}^{2}} \sum_{i=1}^{n} Q_{F}\left(\boldsymbol{\theta} ; \mathbf{Y}_{i}\right),
\end{gathered}
$$

onde

$$
\mathrm{S}^{*}=\mathrm{S}_{10} \beta^{\prime}+\beta \mathrm{S}_{01}+\mathrm{S}_{11} \boldsymbol{\beta} \boldsymbol{\beta}^{\prime}+\boldsymbol{\beta} \boldsymbol{\beta}^{\prime} \mathrm{S}_{11}
$$

$\mathrm{S}_{01}, \mathrm{~S}_{10}$ e $\mathrm{S}_{11}$ são blocos de

$$
\mathbf{S}=\left(\begin{array}{ll}
\mathrm{S}_{00} & \mathrm{~S}_{01} \\
\mathrm{~S}_{10} & \mathrm{~S}_{11}
\end{array}\right)
$$

com as mesmas dimensões dos blocos de $\mathbf{G}$ em (3.16).

Combinando (3.6), (3.10) e (3.11) formamos o logaritmo da função verossimilhança do modelo estrutural. Suas derivadas em relação a $\theta=\left(\boldsymbol{\alpha}^{\prime}, \boldsymbol{\beta}^{\prime}, \sigma_{\epsilon \epsilon}, \mu_{x}, \sigma_{x x}\right)^{\prime}$, agrupadas na matriz

$$
\ddot{\mathbf{L}}_{E}=\left.\frac{\partial^{2} \mathcal{L}(\boldsymbol{\theta} ; \mathbf{Y})}{\partial \boldsymbol{\theta} \partial \boldsymbol{\theta}^{\prime}}\right|_{\boldsymbol{\theta}=\widehat{\theta}}=\left(\begin{array}{ccccc}
\mathbf{L}_{\alpha \alpha} & \mathbf{L}_{\alpha \beta} & \mathbf{L}_{\alpha \sigma_{\epsilon \epsilon}} & \mathbf{L}_{\alpha \mu_{x}} & \mathbf{L}_{\alpha \sigma_{x x}} \\
& \mathbf{L}_{\beta \beta} & \mathbf{L}_{\beta \sigma_{\epsilon \epsilon}} & \mathbf{L}_{\beta \mu_{x}} & \mathbf{L}_{\beta \sigma_{x x}} \\
& & \mathbf{L}_{\sigma_{\epsilon \epsilon} \sigma_{\epsilon \epsilon}} & \mathbf{L}_{\sigma_{\epsilon \epsilon} \mu_{x}} & \mathbf{L}_{\sigma_{\epsilon \epsilon} \sigma_{x x}} \\
& & & \mathbf{L}_{\mu_{x} \mu_{x}} & \mathbf{L}_{\mu_{x} \sigma_{x x}} \\
& & & & \mathbf{L}_{\sigma_{x x} \sigma_{x x}}
\end{array}\right)_{\left.\right|_{\boldsymbol{\theta}=\widehat{\theta}}}
$$

são obtidas de

$$
\begin{aligned}
& \mathbf{L}_{\alpha \alpha}=-\frac{n}{\sigma_{\epsilon \epsilon}}\left(\mathbf{I}_{p}-h \boldsymbol{\beta} \boldsymbol{\beta}^{\prime}\right), \\
& \mathbf{L}_{\alpha \beta}=\overline{y_{0}} \mathbf{L}_{\alpha \alpha}, \quad \mathbf{L}_{\alpha \sigma_{\epsilon \epsilon}}=\mathbf{0} \text {, } \\
& \mathbf{L}_{\alpha \mu_{x}}=-\frac{n(1-h c)}{\sigma_{\epsilon \epsilon}} \boldsymbol{\beta}, \quad \mathbf{L}_{\alpha \sigma_{x x}}=\mathbf{0} \\
& \mathbf{L}_{\beta \beta}=-\frac{n h}{\sigma_{\epsilon \epsilon}}\left[\sigma_{\epsilon \epsilon}\left(\mathbf{I}_{p}-2 h \beta \beta^{\prime}\right)+\frac{{\overline{y_{0}}}^{2}\left(\mathbf{I}_{p}-h \beta \beta^{\prime}\right)}{h}\right. \\
& \left.+h \boldsymbol{b}^{\prime} \mathrm{S} \boldsymbol{b}\left(\mathbf{I}_{p}-4 h \boldsymbol{\beta} \boldsymbol{\beta}^{\prime}\right)-\mathrm{S}_{11}+2 h \mathrm{~S}^{*}\right] \text {, } \\
& \mathbf{L}_{\beta \sigma_{\epsilon \epsilon}}=\frac{n h^{2}}{\sigma_{\epsilon \epsilon}} \boldsymbol{\beta}-\frac{n}{\sigma_{\epsilon \epsilon}^{2}}\left[h\left(\mathbf{S}_{10}+\mathbf{S}_{11} \beta\right)\left(1+\frac{h \sigma_{\epsilon \epsilon}}{\sigma_{x x}}\right)-h^{2} b^{\prime} \mathbf{S} b\left(1+\frac{2 h \sigma_{\epsilon \epsilon}}{\sigma_{x x}}\right) \boldsymbol{\beta}\right], \\
& \mathbf{L}_{\beta \mu_{x}}=\overline{y_{0}} \mathbf{L}_{\alpha \mu_{x}} \\
& \mathbf{L}_{\beta \sigma_{x x}}=-\frac{h n(1-h c)}{\sigma_{x x}}\left[\left(1+\frac{2 h b^{\prime} \mathbf{S} b}{\sigma_{\epsilon \epsilon}}\right) \boldsymbol{\beta}-\frac{1}{\sigma_{\epsilon \epsilon}}\left(\mathrm{S}_{10}+\mathrm{S}_{11} \beta\right)\right],
\end{aligned}
$$




$$
\begin{gathered}
\mathbf{L}_{\sigma_{\epsilon \epsilon} \sigma_{\epsilon \epsilon}}=\frac{n}{\sigma_{\epsilon \epsilon}^{2}}\left[\frac{1}{2}\left(p+(1-h c)^{2}\right)+\frac{h^{2}}{\sigma_{x x}} \boldsymbol{b}^{\prime} \mathbf{S} \boldsymbol{b}\left(1+\frac{\sigma_{\epsilon \epsilon} h}{\sigma_{x x}}\right)-\frac{1}{\sigma_{\epsilon \epsilon}}\left(\operatorname{tr}(\mathbf{S})-h \boldsymbol{b}^{\prime} \mathbf{S} b\right)\right], \\
\mathbf{L}_{\sigma_{\epsilon \epsilon} \mu_{x}}=0 \\
\mathbf{L}_{\sigma_{\epsilon} \sigma_{x x}}=\frac{n h}{2 \sigma_{x x}}\left[\frac{h c}{\sigma_{x x}}-\frac{\boldsymbol{b}^{\prime} \mathbf{S} b}{\sigma_{\epsilon \epsilon}}\left(\frac{1-h c}{\sigma_{\epsilon \epsilon}}-\frac{h(2 h c-1)}{\sigma_{x x}}\right)\right], \\
\mathbf{L}_{\mu_{x} \mu_{x}}=-\frac{n c}{\sigma_{\epsilon \epsilon}}(1-h c), \quad \mathbf{L}_{\mu_{x} \sigma_{x x}}=0 \mathrm{e} \\
\mathbf{L}_{\sigma_{x x} \sigma_{x x}}=n\left(\frac{h c}{\sigma_{x x}}\right)^{2}\left[\frac{1}{2}+\frac{\boldsymbol{b}^{\prime} \mathbf{S} b}{c \sigma_{\epsilon \epsilon}}(h c-1)\right],
\end{gathered}
$$

onde $\mathbf{S}^{*}$ está definida em (3.19).

Ressaltamos que $-\ddot{\mathbf{L}}_{F}$ e $-\ddot{\mathbf{L}}_{E}$ são as matrizes de informação observadas.

\subsection{O modelo estrutural heteroscedástico}

Em (3.5) a matriz de covariância dos erros de medição é $\boldsymbol{\Sigma}_{\epsilon \epsilon}=\mathbf{D}\left(\boldsymbol{\sigma}_{\epsilon \epsilon}\right)$, de modo que

$$
\mathrm{V}=\sigma_{x x}\left(\begin{array}{l}
1 \\
\boldsymbol{\beta}
\end{array}\right)\left(\begin{array}{l}
1 \\
\boldsymbol{\beta}
\end{array}\right)^{\prime}+\mathrm{D}\left(\boldsymbol{\sigma}_{\epsilon \epsilon}\right)
$$

Apesar da formulação (3.3) incluir parâmetros de interesse como os vícios dos instrumentos $(\boldsymbol{\alpha}$ e $\boldsymbol{\beta})$, essa parametrização não é a mais apropriada à estimação dos parâmetros. Theobald \& Mallinson (1978) propõem reparametrizar (3.3) por intermédio de Análise Fatorial. Seja $x_{i}=\mu_{x}+\sqrt{\sigma_{x x}} f_{i}, \operatorname{com} f_{i} \underset{\mathrm{iid}}{\sim} \mathrm{N}(0,1), i=1, \ldots, n$. Substituindo em (3.3) resulta

$$
\begin{aligned}
& \mathbf{Y}_{i}=\boldsymbol{\mu}+\lambda f_{i}+\epsilon_{i}, \\
& \mathbf{Y}_{i} \stackrel{\text { iid }}{\sim} \mathrm{N}_{r}(\boldsymbol{\mu}, \mathbf{V}),
\end{aligned}
$$

$i=1, \ldots, n$, onde

$$
\boldsymbol{\mu}=\left(\begin{array}{c}
0 \\
\alpha
\end{array}\right)+\left(\begin{array}{l}
1 \\
\beta
\end{array}\right) \mu_{x}, \quad \boldsymbol{\lambda}=\sqrt{\sigma_{x x}}\left(\begin{array}{l}
1 \\
\beta
\end{array}\right) \quad \text { e } \quad \mathrm{V}=\boldsymbol{\lambda} \boldsymbol{\lambda}^{\prime}+\mathbf{D}\left(\boldsymbol{\sigma}_{\epsilon \epsilon}\right) .
$$

O vetor de parâmetros do modelo passa a ser $\boldsymbol{\gamma}=\left(\boldsymbol{\mu}^{\prime}, \boldsymbol{\lambda}^{\prime}, \boldsymbol{\sigma}_{\epsilon \epsilon}^{\prime}\right)^{\prime}$, notando que

$$
\begin{gathered}
\alpha=\left(\mu_{1}, \ldots, \mu_{p}\right)^{\prime}-\mu_{0}\left(\lambda_{1}, \ldots, \lambda_{p}\right)^{\prime} / \lambda_{0}, \\
\beta=\left(\lambda_{1}, \ldots, \lambda_{p}\right)^{\prime} / \lambda_{0}, \\
\mu_{x}=\mu_{0} \quad \text { e } \quad \sigma_{x x}=\lambda_{0}^{2} .
\end{gathered}
$$




\subsubsection{EMV}

Na situação $p=2$ (instrumento de referência e dois outros instrumentos) Barnett (1969) apresenta expressões de estimadores pelo método dos momentos, podendo ocorrer estimativas negativas de variâncias. Carter (1981) supera este inconveniente usando estimação de MV restrita. Para $p>2$ até o presente momento desconhecemos as expressões dos EMV dos parâmetros. Barnett (1969) sugere estimadores pelo método dos momentos. Theobald \& Mallinson (1978) recorrem a programas computacionais desenvolvidos para estimação por MV nos modelos de Análise Fatorial, dos quais (3.21) é um caso particular. Fuller (1987, Capítulo 4) descreve procedimentos iterativos de estimação por MV no modelo de Análise Fatorial. Bolfarine \& Galea-Rojas (1995) propõem estimar os parâmetros através do algoritmo EM (Dempster, Laird \& Rubin, 1977). As expressões resultantes são simples, facilmente programáveis. Adotaremos esta linha.

Inicialmente fazemos $\boldsymbol{z}_{i}=\left(f_{i}, \mathbf{Y}_{i}^{\prime}\right)^{\prime}$, de modo que

$$
z_{1}, \ldots, z_{n} \stackrel{\text { iid }}{\sim} \mathrm{N}_{p+2}\left(\boldsymbol{\mu}_{z}, \Sigma_{z z}\right)
$$

onde

$$
\boldsymbol{\mu}_{z}=\left(0, \boldsymbol{\mu}^{\prime}\right)^{\prime} \quad \text { e } \quad \Sigma_{z z}=\left(\begin{array}{cc}
1 & \lambda^{\prime} \\
& \lambda \lambda^{\prime}+\mathrm{D}\left(\boldsymbol{\sigma}_{\epsilon \epsilon}\right)
\end{array}\right) .
$$

De acordo com Dempster et al. (1977), $\boldsymbol{z}_{i}$ é o vetor de dados aumentados (em relação a $\mathbf{Y}_{i}$ ) pela variável inobservável $f_{i}, i=1, \ldots, n$. Uma iteração do algoritmo é composta de dois passos. No passo "E", com as estimativas de $\gamma$, é calculada a esperança condicional das estatísticas suficientes da distribuição das variáveis $\left(\boldsymbol{z}_{1}^{\prime}, \ldots, \boldsymbol{z}_{n}^{\prime}\right)^{\prime}$ dado $\mathrm{Y}$. Neste modelo isto se reduz a

$$
\begin{aligned}
& \widehat{f_{i}}=\mathrm{E}(f i \mid \mathbf{Y} ; \boldsymbol{\gamma})=\frac{1}{c_{*}} \sum_{j=0}^{p} \frac{\lambda_{j}}{\sigma_{\epsilon \epsilon j}}\left(y_{i j}-\mu_{j}\right), \\
& \widehat{f_{i}^{2}}=\mathrm{E}\left(f i^{2} \mid \mathbf{Y} ; \gamma\right)=\frac{1}{c_{*}}+\widehat{f}_{i}^{2},
\end{aligned}
$$

$i=1, \ldots, n$, onde

$$
c_{*}=1+\sum_{j=0}^{p} \frac{\lambda_{j}^{2}}{\sigma_{\epsilon \epsilon j}} .
$$

No passo "M" a verossimilhança de (3.23) é maximizada substituindo as estatísticas suficientes pelos preditores avaliados no passo "E". A Figura 3.1 contém um esboço 
início

$\widehat{\boldsymbol{\mu}}=\overline{\mathbf{Y}}=\sum_{i=1}^{n} \mathbf{Y}_{i} / n$,

atribuir valores iniciais a $\boldsymbol{\lambda}$ e $\sigma_{\epsilon \epsilon}\left(\widehat{\boldsymbol{\lambda}}\right.$ e $\left.\widehat{\boldsymbol{\sigma}_{\epsilon \epsilon}}\right)$,

passo E:

$$
\begin{aligned}
& \widehat{c_{*}}=1+\sum_{j=0}^{p} \frac{{\widehat{\lambda_{j}}}^{2}}{\widehat{\sigma_{\epsilon \epsilon j}}}, \\
& \widehat{f_{i}}=\frac{1}{\widehat{c_{*}}} \sum_{j=0}^{p} \frac{\widehat{\lambda_{j}}}{\widehat{\sigma_{\epsilon \epsilon j}}}\left(y_{i j}-\widehat{\mu_{j}}\right), \\
& \widehat{f_{i}^{2}}=\frac{1}{\widehat{c_{*}}}+\widehat{f}_{i}^{2}, i=1, \ldots, n,
\end{aligned}
$$

passo M:

$$
\begin{aligned}
& \widehat{\lambda_{j}}=\frac{\sum_{i=1}^{n} \widehat{f_{i}}\left(y_{i j}-\widehat{\mu_{j}}\right)}{\sum_{i=1}^{n} \widehat{f_{i}^{2}}}, \\
& \widehat{\sigma_{\epsilon \epsilon j}}=\frac{1}{n} \sum_{i=1}^{n}\left[\left(y_{i j}-\widehat{\mu_{j}}\right)-\widehat{\lambda_{j} \widehat{f}_{i}}\right]^{2}+\frac{{\widehat{\lambda_{j}}}^{2}}{\widehat{c_{*}}}, j=0,1, \ldots, p,
\end{aligned}
$$

repetir passos E e M,

fim.

Figura 3.1: Algoritmo EM no modelo estrutural heteroscedástico. 
do esquema iterativo. A cada iteração do algoritmo EM o valor do função verossimilhança no modelo (3.21) não diminui. Enfatizamos que a maximização do passo "M" é direta, sem necessidade de procedimentos iterativos. As estimativas iniciais de $\lambda \mathrm{e}$ $\sigma_{\epsilon \epsilon}$ são baseadas nos estimadores consistentes calculados pelo método dos momentos (Barnett, 1969) considerando todos os subconjuntos de três instrumentos, conforme descrito em Galea-Rojas (1995). No passo "M" temos assegurado que $\widehat{\sigma_{\epsilon \epsilon j}} \geq 0$, $j=0,1, \ldots, p$. Os ciclos se repetem até que algum critério de parada - baseado nas diferenças das estimativas dos parâmetros entre duas iterações sucessivas ou no número máximo de iterações - seja atingido.

\subsubsection{Matriz de informação observada}

Escrevemos o logaritmo da função verossimilhança do modelo (3.21) como

$$
\mathcal{L}(\boldsymbol{\gamma} ; \mathbf{Y})=\sum_{i=1}^{n} l\left(\gamma ; \mathbf{Y}_{i}\right)
$$

onde

$$
l\left(\boldsymbol{\gamma} ; \mathbf{Y}_{i}\right)=-\frac{r}{2} \log 2 \pi-\frac{1}{2} \log |\mathbf{V}|-\frac{1}{2}\left(\mathbf{Y}_{i}-\boldsymbol{\mu}\right)^{\prime} \mathbf{V}^{-1}\left(\mathbf{Y}_{i}-\boldsymbol{\mu}\right),
$$

$i=1, \ldots, n, \mathrm{com}$

$$
\log |\mathbf{V}|=\log \left(c_{*}\left|\mathbf{D}\left(\boldsymbol{\sigma}_{\epsilon \epsilon}\right)\right|\right)=\log c_{*}+\sum_{j=0}^{p} \log \sigma_{\epsilon \epsilon j},
$$

com $c_{*}$ definido em (3.24) e

$$
\mathrm{V}^{-1}=\mathrm{D}^{-1}\left(\sigma_{\epsilon \epsilon}\right)-\frac{1}{c_{*}} \mathrm{D}^{-1}\left(\sigma_{\epsilon \epsilon}\right) \lambda \lambda^{\prime} \mathrm{D}^{-1}\left(\sigma_{\epsilon \epsilon}\right) .
$$

A matriz de derivadas de segunda ordem de $\mathcal{L}(\boldsymbol{\gamma} ; \mathbf{Y})$ em relação a $\boldsymbol{\gamma}=\left(\boldsymbol{\mu}^{\prime}, \boldsymbol{\lambda}^{\prime}, \boldsymbol{\sigma}_{\epsilon \epsilon}^{\prime}\right)^{\prime}$, denotada por $\ddot{\mathbf{L}}_{E \gamma}$, é calculada a partir de (3.25):

$$
\ddot{\mathrm{L}}_{E \gamma}=\left.\frac{\partial^{2} \mathcal{L}(\boldsymbol{\gamma} ; \mathbf{Y})}{\partial \gamma \partial \gamma^{\prime}}\right|_{\gamma=\widehat{\gamma}}=\left.\sum_{i=1}^{n} \frac{\partial^{2}}{\partial \gamma \gamma^{\prime}} l\left(\boldsymbol{\gamma} ; \mathbf{Y}_{i}\right)\right|_{\gamma=\hat{\gamma}}=\left(\begin{array}{ccc}
\mathbf{L}_{\mu \mu} & 0 & 0 \\
& \mathrm{~L}_{\lambda \lambda} & \mathbf{L}_{\lambda \sigma_{\epsilon \epsilon}} \\
& \mathbf{L}_{\sigma_{\epsilon \epsilon} \sigma_{\epsilon \epsilon}}
\end{array}\right)_{\left.\right|_{\gamma=\widehat{\gamma}}}
$$

onde $\widehat{\gamma}$ é calculado pelo algoritmo EM (Figura 3.1),

$$
\mathbf{L}_{\mu \mu}=-n \mathbf{V}^{-1}
$$




$$
\begin{aligned}
\mathbf{L}_{\lambda \lambda}= & -n c_{*}^{-1}\left(\mathbf{V}^{-1}-c_{*}^{-1} \mathbf{M}\right)-\frac{n}{2}\left[-2 c_{*}^{-1} \mathbf{D}^{-1}\left(\boldsymbol{\sigma}_{\epsilon \epsilon}\right) \mathbf{S D}^{-1}\left(\sigma_{\epsilon \epsilon}\right)+4 c_{*}^{-2} \mathbf{D}^{-1}\left(\boldsymbol{\sigma}_{\epsilon \epsilon}\right) \mathbf{S M}\right. \\
& \left.+2 c_{*}^{-2} \operatorname{tr}(\mathbf{S M})\left(\mathbf{V}^{-1}-3 c_{*}^{-1} \mathbf{M}\right)+4 c_{*}^{-2} \mathbf{M S D}^{-1}\left(\sigma_{\epsilon \epsilon}\right)\right] \\
\mathbf{L}_{\lambda \sigma_{\epsilon \epsilon}}= & n c_{*}^{-1} \mathbf{V}^{-1} \mathbf{D}^{-1}\left(\boldsymbol{\sigma}_{\epsilon \epsilon}\right) \mathbf{D}(\boldsymbol{\lambda})-\frac{n}{2}\left[2 c_{*}^{-1} \mathbf{D}^{-1}\left(\boldsymbol{\sigma}_{\epsilon \epsilon}\right) \mathbf{S D}(\boldsymbol{\lambda}) \mathbf{D}^{-2}\left(\boldsymbol{\sigma}_{\epsilon \epsilon}\right)-2 c_{*}^{-2} \operatorname{tr}(\mathbf{S M})\right. \\
& \left.\times \mathbf{D}^{-2}\left(\boldsymbol{\sigma}_{\epsilon \epsilon}\right) \mathbf{D}(\boldsymbol{\lambda})+4 c_{*}^{-3} \operatorname{tr}(\mathbf{S M}) \mathbf{M D}^{-1}\left(\boldsymbol{\sigma}_{\epsilon \epsilon}\right) \mathbf{D}(\boldsymbol{\lambda})-4 c_{*}^{-2} \mathbf{M S D}(\boldsymbol{\lambda}) \mathbf{D}^{-2}\left(\boldsymbol{\sigma}_{\epsilon \epsilon}\right)\right]
\end{aligned}
$$

e

$$
\begin{aligned}
\mathbf{L}_{\sigma_{\epsilon \epsilon} \sigma_{\epsilon \epsilon}}= & -\frac{n}{2}\left[-\mathbf{D}^{-2}\left(\sigma_{\epsilon \epsilon}\right)+2 c_{*}^{-1} \mathbf{D}^{-3}\left(\boldsymbol{\sigma}_{\epsilon \epsilon}\right) \mathbf{D}^{2}(\boldsymbol{\lambda})\right. \\
& -c_{*}^{-2} \mathbf{D}^{-1}\left(\boldsymbol{\sigma}_{\epsilon \epsilon}\right) \mathbf{D}(\boldsymbol{\lambda}) \mathbf{M D}(\boldsymbol{\lambda}) \mathbf{D}^{-1}\left(\boldsymbol{\sigma}_{\epsilon \epsilon}\right)+2 \mathbf{D}^{-3}\left(\boldsymbol{\sigma}_{\epsilon \epsilon}\right) \mathbf{D}\left(\mathrm{S}_{00}, \mathrm{~S}_{11}, \ldots, \mathrm{S}_{p p}\right) \\
& -4 c_{*}^{-1} \mathbf{D}^{-3}\left(\boldsymbol{\sigma}_{\epsilon \epsilon}\right) \mathbf{D}(\boldsymbol{\lambda}) \mathbf{D}\left(\mathbf{S D}^{-1}\left(\boldsymbol{\sigma}_{\epsilon \epsilon}\right) \boldsymbol{\lambda}\right)-2 c_{*}^{-1} \mathbf{D}^{-2}\left(\boldsymbol{\sigma}_{\epsilon \epsilon}\right) \mathbf{D}(\boldsymbol{\lambda}) \mathbf{S D}(\boldsymbol{\lambda}) \mathbf{D}^{-2}\left(\boldsymbol{\sigma}_{\epsilon \epsilon}\right) \\
& +2 c_{*}^{-2} \operatorname{tr}(\mathbf{S M}) \mathbf{D}^{-3}\left(\boldsymbol{\sigma}_{\epsilon \epsilon}\right) \mathbf{D}^{2}(\boldsymbol{\lambda})-2 c_{*}^{-3} \operatorname{tr}(\mathbf{S M}) \mathbf{D}^{-1}\left(\boldsymbol{\sigma}_{\epsilon \epsilon}\right) \mathbf{D}(\boldsymbol{\lambda}) \mathbf{M D}(\boldsymbol{\lambda}) \mathbf{D}^{-1}\left(\boldsymbol{\sigma}_{\epsilon \epsilon}\right) \\
& \left.+2 c_{*}^{-2} \mathbf{D}^{-1}\left(\boldsymbol{\sigma}_{\epsilon \epsilon}\right) \mathbf{D}(\boldsymbol{\lambda}) \mathbf{M S D}(\boldsymbol{\lambda}) \mathbf{D}^{-2}\left(\boldsymbol{\sigma}_{\epsilon \epsilon}\right)\right]
\end{aligned}
$$

onde

$$
\mathbf{M}=\mathbf{D}^{-1}\left(\sigma_{\epsilon \epsilon}\right) \lambda \lambda^{\prime} \mathbf{D}^{-1}\left(\sigma_{\epsilon \epsilon}\right)
$$

e "tr" indicando o traço de uma matriz, com $c_{*}, \mathrm{~V}^{-1}$ e $\mathbf{S}$ segundo (3.24), (3.26) e (3.15), respectivamente. Estas expressões particularizam resultados de Galea-Rojas, Vilca-Labra \& Bolfarine (2001).

O estudo de influência local (seções 3.4 e 3.5) será desenvolvido na parametrização de regressão $(\boldsymbol{\theta})$. Denotando por $\ddot{\mathbf{L}}_{E}$ a matriz de derivadas de segunda ordem de $\mathcal{L}(\boldsymbol{\theta} ; \mathbf{Y})$ em relação a $\boldsymbol{\theta}$, temos

$$
\ddot{\mathbf{L}}_{E}=\left(\mathbf{J}_{\gamma \theta} \ddot{\mathbf{L}}_{E \gamma} \mathbf{J}_{\gamma \theta}^{\prime}\right)_{\mid \gamma=\hat{\gamma}, \theta=\hat{\theta}}
$$

onde

$$
\mathbf{J}_{\gamma \theta}=\left(\begin{array}{cccc}
0 & \mathbf{I}_{p} & 0 & 0 \\
0 & \mu_{x} \mathbf{I}_{p} & \left(0, \sqrt{\sigma_{x x}} \mathbf{I}_{p}\right) & 0 \\
0 & 0 & 0 & \mathbf{I}_{p+1} \\
1 & \beta^{\prime} & 0 & 0 \\
0 & 0 & \frac{1}{2 \sqrt{\sigma_{x x}}}\left(1, \boldsymbol{\beta}^{\prime}\right) & 0
\end{array}\right)_{\boldsymbol{\theta}=\hat{\boldsymbol{\theta}}}
$$

é a matriz $3 r \times 3 r$ tendo elemento geral $\partial \gamma_{j} / \partial \theta_{k}, j, k=0,1, \ldots, p$, e lembrando que (3.21) e (3.22) efetuam as mudanças de parametrização. 


\subsection{Influência local}

Nesta seção resumidamente expomos a metodologia de análise de influência local à maneira de Cook (1986) (vide também Cook, 1987, e a apresentação detalhada em de Souza, 1999).

Cook (1986) modifica o modelo através de um vetor de perturbações $\omega, \omega \in \Omega \subseteq$ $\mathbb{R}^{q}, \Omega$ aberto. O objetivo é comparar os modelos postulado e perturbado fazendo $\omega$ variar em $\Omega$, procurando elementos em $\omega$ responsáveis pelo afastamento entre os modelos. Para tanto, devemos selecionar os resultados a serem monitorados, os esquemas de perturbação e um método de comparação entre os dois modelos. Estamos interessados em detectar variações nos estimadores de MV dos parâmetros provocadas pelos esquemas das seções 3.5.1, 3.5.2 e 3.5.3.

Seja $\mathcal{L}(\theta ; \mathbf{Y})$ o logaritmo da função verossimilhança de um dos modelos propostos (seções 3.2 e 3.3). Aqui, $\boldsymbol{\theta} \in \Theta$ designa genericamente o vetor de parâmetros, independentemente da parametrização adotada. Seja $\mathcal{L}^{*}(\theta ; \omega, \mathbf{Y})$ o logaritmo da função verossimilhança do modelo perturbado; por hipótese, duas vezes continuamente diferenciável em $\left(\theta^{\prime}, \omega^{\prime}\right)^{\prime}$. Adicionalmente supomos a existência de $\omega_{0} \in \Omega$ satisfazendo $\mathcal{L}^{*}\left(\theta ; \omega_{0}, \mathbf{Y}\right)=\mathcal{L}(\theta ; \mathbf{Y})$.

A influência das perturbações sobre os estimadores de MV de $\theta$ pode ser quantificada pelo afastamento da verossimilhança, definido como

$$
L D(\boldsymbol{\omega})=2\left[\mathcal{L}(\widehat{\boldsymbol{\theta}} ; \mathbf{Y})-\mathcal{L}\left(\widehat{\boldsymbol{\theta}}_{\omega} ; \mathbf{Y}\right)\right]
$$

onde $\widehat{\theta}$ e $\widehat{\theta}_{\omega}$ denotam os estimadores de MV de $\theta$ sob os modelos postulado e perturbado, respectivamente, notando que $L D \geq 0$ e $L D\left(\omega_{0}\right)=0$. Cook (1986) investiga a influência local examinando o comportamento de $L D$ mediante pequenas perturbações de $\omega_{0}$. Isto pode ser efetuado explorando a geometria da superfície $\Lambda$ em $\mathbb{R}^{q+1}$ formada pelos vetores $\left(\boldsymbol{\omega}^{\prime}, L D(\omega)\right)^{\prime}, \boldsymbol{\omega} \in \boldsymbol{\Omega}$. Inicialmente selecionamos uma direção unitária $\boldsymbol{l}$ em $\Omega$, que determina uma reta $\omega(a)$ em $\Omega$ passando por $\omega_{0}$,

$$
\omega(a)=\omega_{0}+a l, a \in \mathbb{R} .
$$

As variações em $L D(\omega)$ são estudadas através da curvatura das seções normais a $\Lambda$ passando por $\left(\omega_{0}^{\prime}, L D\left(\omega_{0}\right)\right)^{\prime}$, que se reduz à curvatura da curva plana $(a, L D(\omega(a))$, $a \in \mathbb{R}$. De particular utilidade são a curvatura máxima $\left(C_{l_{\max }}\right)$ e a direção correspondente $\left(\boldsymbol{l}_{\max }\right) . C_{l_{\max }}$ tem valor relativo, enquanto o gráfico de índices (index plot) 
de $l_{\max }$ revela possíveis elementos influentes. Na Seção 3.6 ilustramos esses pontos. Cook mostra que $C_{l_{\max }}$ é o maior (em valor absoluto) autovalor de

$$
\left(\Delta^{\prime} \ddot{\mathbf{L}}^{-1} \Delta\right)_{\mid \omega=\omega_{0}, \theta=\hat{\theta}}
$$

onde $\Delta$ é a matriz $p^{*} \times q$ com elemento geral $\frac{\partial^{2}}{\partial \omega_{j} \partial \theta_{i}} \mathcal{L}^{*}(\theta ; \omega, \mathbf{Y}), i=1, \ldots, p^{*}, j=$ $1, \ldots, q, p^{*}$ é o número de parâmetros do modelo e $-\ddot{\mathbf{L}}$ é a matriz de informação de Fisher observada no modelo postulado $\left(\omega=\omega_{0}\right) ; p^{*}$ e $\ddot{\mathbf{L}}$ dependendo do modelo (seções 3.2 e 3.3 ). A direção $l_{\max }$ é o autovetor associado a $C_{l_{\max }}$.

Em certas situações podemos estar interessados na influência de perturbações sobre os estimadores de MV de $\theta_{1}$, um subvetor de $\theta=\left(\theta_{1}^{\prime}, \theta_{2}^{\prime}\right)^{\prime}$. O afastamento da verossimilhança passa a ser

$$
L D_{1}(\boldsymbol{\omega})=2\left[\mathcal{L}(\widehat{\boldsymbol{\theta}} ; \mathbf{Y})-\mathcal{L}\left(\widehat{\boldsymbol{\theta}}_{1 \omega}, \widehat{\boldsymbol{\theta}}_{2}\left(\widehat{\boldsymbol{\theta}}_{1 \omega}\right) ; \mathbf{Y}\right)\right]
$$

onde $\widehat{\boldsymbol{\theta}}_{1 \omega}$ é obtido da partição $\widehat{\boldsymbol{\theta}}_{\omega}=\left(\widehat{\boldsymbol{\theta}}_{1 \omega}^{\prime}, \widehat{\boldsymbol{\theta}}_{2 \omega}^{\prime}\right)^{\prime}$ e $\widehat{\boldsymbol{\theta}}_{2}\left(\widehat{\boldsymbol{\theta}}_{1 \omega}\right)$ maximiza $\mathcal{L}\left(\widehat{\boldsymbol{\theta}}_{1 \omega}, \boldsymbol{\theta}_{2} ; \mathbf{Y}\right)$. A curvatura $C_{l_{\max }}$ e a direção $\boldsymbol{l}_{\max }$ são calculadas usando (3.31) substituindo $\ddot{\mathbf{L}}$ por

$$
\ddot{\mathbf{L}}-\left(\begin{array}{cc}
0 & 0 \\
& \ddot{\mathbf{L}}_{22}^{-1}
\end{array}\right)
$$

onde $\ddot{\mathbf{L}}_{22}$ é extraída de $\ddot{\mathbf{L}}$ subdividida de acordo com a partição de $\boldsymbol{\theta}$.

Em síntese, na metodologia de Cook a curvatura $C_{l_{\max }}$, a direção $\boldsymbol{l}_{\max }$ correspondente e o traçado de $L D\left(\omega_{0}+a l_{\max }\right)$ versus $a$ são as principais ferramentas de diagnóstico.

\subsection{Esquemas de perturbação}

Diferentes maneiras de perturbar os modelos das seções 3.2 e 3.3 são examinadas. Concretamente, apresentamos a matriz $\Delta$ presente em (3.31) para cada esquema e cada modelo. No modelo homoscedástico recorremos em parte a Galea-Rojas, Bolfarine \& de Castro (1999) e no modelo heteroscedástico particularizamos resultados de Galea-Rojas et al. (2001).

Mencionamos que o modelo de calibração estrutural faz parte da classe dos modelos com erros nas variáveis multivariados. Entretanto, existem esquemas de perturbação inerentes ao modelo de calibração e que merecem estudo separado (seções 3.5 .2 e 3.5 .3$)$. 


\subsubsection{Perturbação da ponderação dos casos}

Tomamos um vetor $\boldsymbol{\omega} n \times 1(q=n)$ de pesos aplicados às contribuições das observações no cálculo do logaritmo da função verossimilhança, generalizando a situação em que a $i$-ésima observação é incluída $\left(\omega_{i}=1\right)$ ou excluída $\left(\omega_{i}=0\right)$ deste cálculo, $i=1, \ldots, n$. O vetor $\omega_{0}=1_{n}$ reflete ausência de perturbação.

\section{Modelo homoscedástico}

O logaritmo da função verossimilhança perturbada tem expressão

$$
\mathcal{L}^{*}(\boldsymbol{\theta} ; \boldsymbol{\omega}, \mathbf{Y})=\sum_{i=1}^{n} \omega_{i} l\left(\boldsymbol{\theta} ; \mathbf{Y}_{i}\right)
$$

No modelo funcional, $\boldsymbol{\theta}=\left(\boldsymbol{\alpha}^{\prime}, \boldsymbol{\beta}^{\prime}, \sigma_{\epsilon \epsilon}\right)^{\prime}$ e $l\left(\boldsymbol{\theta} ; \mathbf{Y}_{i}\right)$ segue de (3.7) e (3.14), ao passo que no modelo estrutural, $\theta=\left(\boldsymbol{\alpha}^{\prime}, \boldsymbol{\beta}^{\prime}, \sigma_{\epsilon \epsilon}, \mu_{x}, \sigma_{x x}\right)^{\prime}$ e $l\left(\boldsymbol{\theta} ; \mathbf{Y}_{i}\right)$ vem de (3.10). Sejam $\Delta_{F}$ e $\Delta_{E}$ as matrizes de derivadas de segunda ordem de $\mathcal{L}^{*}$ em relação a $\theta$ e $\omega^{\prime}$ nos modelos funcional e estrutural, respectivamente, organizadas em blocos com

$$
\Delta_{F}=\left(\begin{array}{c}
\Delta_{\alpha} \\
(p \times n) \\
\Delta_{\beta} \\
(p \times n) \\
\Delta_{\sigma_{\epsilon \epsilon}} \\
(1 \times n)
\end{array}\right), \quad \Delta_{E}=\left(\begin{array}{c}
\Delta_{\alpha} \\
\Delta_{\beta} \\
\Delta_{\sigma_{\epsilon \epsilon}} \\
\Delta_{\mu_{x}} \\
(1 \times n) \\
\Delta_{\sigma_{x x}} \\
(1 \times n)
\end{array}\right) \quad \mathrm{e}
$$

$\boldsymbol{\Delta}_{\alpha}=\left(\boldsymbol{\Delta}_{\alpha 1}, \ldots, \boldsymbol{\Delta}_{\alpha n}\right)$ (as demais matrizes são definidas analogamente). Após algumas manipulações algébricas chegamos a

$$
\begin{gathered}
\Delta_{\alpha i}= \begin{cases}\frac{1}{\sigma_{\epsilon \epsilon}}\left[\left(\mathbf{Y}_{1 i}-\overline{\mathbf{Y}}_{1}\right)-\left(x_{i}-\overline{y_{0}}\right) \boldsymbol{\beta}\right], & \text { para o modelo funcional, } \\
\frac{1}{\sigma_{\epsilon \epsilon}}\left[\left(\mathbf{Y}_{1 i}-\overline{\mathbf{Y}}_{1}\right)-h q_{2 i} \boldsymbol{\beta}\right], & \text { para o modelo estrutural, }\end{cases} \\
\Delta_{\beta i}= \begin{cases}x_{i} \Delta_{\alpha i}, & \text { no modelo funcional, } \\
-h \boldsymbol{\beta}+\left(\overline{y_{0}}+h q_{2 i}\right) \Delta_{\alpha i}, & \text { no modelo estrutural, }\end{cases} \\
\Delta_{\sigma_{\epsilon \epsilon} i}= \begin{cases}-\frac{1}{2 \sigma_{\epsilon \epsilon}}\left[r-Q_{F}\left(\theta ; \mathbf{Y}_{i}\right)\right], & \text { no modelo funcional, } \\
-\frac{1}{2}\left(\frac{p}{\sigma_{\epsilon \epsilon}}+\frac{h}{\sigma_{x x}}\right)-\frac{1}{2 \sigma_{\epsilon \epsilon}}\left[\frac{h^{2}}{\sigma_{x x}} q_{2 i}^{2}-Q_{E}\left(\theta ; \mathbf{Y}_{i}\right)\right], & \text { no modelo estrutural, }\end{cases}
\end{gathered}
$$




$$
\begin{gathered}
\Delta_{\mu_{x i}}=\left(\frac{1-h c}{\sigma_{\epsilon \epsilon}}\right) q_{2 i}, \\
\Delta_{\sigma_{x x i}}=-\frac{h}{2 \sigma_{x x}}\left(c-q_{2 i} \Delta_{\mu_{x i}}\right),
\end{gathered}
$$

$i=1, \ldots, n$. Nestas expressões substituímos $x_{i}, q_{2 i}, Q_{F}, h$ e $Q_{E}$ por (3.13), (3.12) e (3.11), nesta ordem, calculadas nos estimadores de MV. O vetor $\mathbf{Y}_{i}$ está definido em (3.8), $\mathbf{Y}_{1 i}$ e $\overline{y_{0}}$ são obtidos de (3.17).

Havendo interesse específico na influência sobre os estimadores de MV de $\theta_{1}=$ $\left(\boldsymbol{\alpha}^{\prime}, \boldsymbol{\beta}^{\prime}\right)^{\prime}-$ vícios aditivos $(\boldsymbol{\alpha})$ e multiplicativos $(\boldsymbol{\beta})$-, no modelo funcional a expressão (3.32) se iguala a

$$
\left(\begin{array}{ll}
\mathbf{L}_{\alpha \alpha} & \mathbf{L}_{\alpha \beta} \\
& \mathbf{L}_{\beta \beta}
\end{array}\right)^{-1} \quad \begin{aligned}
& 0 \\
&
\end{aligned}
$$

pois $\ddot{\mathbf{L}}_{F}$ em (3.18) é diagonal em blocos.

\section{Modelo estrutural heteroscedástico}

Recordando a Seção 3.3, trabalhamos com a parametrização de fatores, traduzida por $\boldsymbol{\gamma}=\left(\boldsymbol{\mu}^{\prime}, \boldsymbol{\lambda}^{\prime}, \boldsymbol{\sigma}_{\epsilon \epsilon}^{\prime}\right)^{\prime}$, conforme (3.21), e o logaritmo da função verossimilhança perturbada torna-se

$$
\mathcal{L}^{*}(\gamma ; \omega, \mathbf{Y})=\sum_{i=1}^{n} \omega_{i} l\left(\gamma ; \mathbf{Y}_{i}\right)
$$

onde $l\left(\gamma ; \mathbf{Y}_{i}\right)$ vem de (3.25). Derivando $\mathcal{L}^{*}$ em relação a $\gamma$ e $\omega^{\prime}$ e calculando em $\omega=\omega_{0}=\mathbf{1}_{n}$ formamos a matriz

$$
\Delta_{E \gamma}=\left(\begin{array}{c}
\Delta_{\mu} \\
(r \times n) \\
\Delta_{\lambda} \\
(r \times n) \\
\Delta_{\sigma_{\epsilon \epsilon}} \\
(r \times n)
\end{array}\right),
$$

com $\Delta_{\mu}=\left(\Delta_{\mu 1}, \ldots, \Delta_{\mu n}\right) ; \Delta_{\lambda}$ e $\Delta_{\sigma_{\epsilon \epsilon}}$ são análogas. Fazendo

$$
\mathrm{W}_{i}=\mathrm{Y}_{i}-\boldsymbol{\mu}
$$

operações algébricas conduzem a

$$
\Delta_{\mu i}=\mathrm{V}^{-1} \mathbf{W}_{i}
$$




$$
\begin{aligned}
\Delta_{\lambda i}= & \frac{1}{c_{*}}\left[2\left(\mathbf{W}_{i}^{\prime} \mathbf{D}^{-1}\left(\sigma_{\epsilon \epsilon}\right) \boldsymbol{\lambda}\right) \mathbf{V}^{-1}+\mathbf{D}^{-1}\left(\sigma_{\epsilon \epsilon}\right) \mathbf{W}_{i} \boldsymbol{\lambda}^{\prime} \mathbf{D}^{-1}\left(\sigma_{\epsilon \epsilon}\right)\right. \\
& \left.-\left(\mathbf{W}_{i}^{\prime} \mathbf{D}^{-1}\left(\boldsymbol{\sigma}_{\epsilon \epsilon}\right) \boldsymbol{\lambda}\right) \mathbf{D}^{-1}\left(\boldsymbol{\sigma}_{\epsilon \epsilon}\right)\right]
\end{aligned}
$$

e

$$
\begin{aligned}
\Delta_{\sigma_{\epsilon \epsilon i}}= & -\frac{1}{2} \mathbf{D}^{-1}\left(\boldsymbol{\sigma}_{\epsilon \epsilon}\right)\left[\mathbf{1}_{r}-\frac{1}{c_{*}} \mathbf{D}^{-1}\left(\boldsymbol{\sigma}_{\epsilon \epsilon}\right) \mathbf{D}(\boldsymbol{\lambda}) \boldsymbol{\lambda}\right]-\frac{1}{2} \mathbf{D}^{-2}\left(\boldsymbol{\sigma}_{\epsilon \epsilon}\right) \\
& \times\left[-\mathbf{D}\left(\mathbf{W}_{i}\right) \mathbf{W}_{i}+\frac{2}{c_{*}} \mathbf{D}(\boldsymbol{\lambda}) \mathbf{W}_{i} \mathbf{W}_{i}^{\prime} \mathbf{D}^{-1}\left(\sigma_{\epsilon \epsilon}\right) \lambda-\frac{1}{c_{*}^{2}}\left(\mathbf{W}_{i}^{\prime} \mathbf{M W}_{i}\right) \mathbf{D}(\boldsymbol{\lambda}) \boldsymbol{\lambda}\right],
\end{aligned}
$$

$i=1, \ldots, n$, onde $\mathbf{V}^{-1}, c_{*}$ e $\mathbf{M}$ se encontram em (3.26), (3.24) e (3.28). Retornando à parametrização de regressão temos

$$
\Delta_{E}=\mathbf{J}_{\gamma \theta} \Delta_{E \gamma}
$$

sendo que $\boldsymbol{\Delta}_{E}$ está em (3.33) e $\mathbf{J}_{\gamma \theta}$ é dada por (3.30). Ressaltamos que na expressão (3.31) tanto podemos usar $\Delta_{E \gamma}$ vista acima e $\ddot{\mathbf{L}}_{E}$ (3.27) ou $\Delta_{E}$ e $\ddot{\mathbf{L}}_{E}$ (3.29). Por outro lado, se estivermos interessados nos vícios $\theta_{1}=\left(\alpha^{\prime}, \beta^{\prime}\right)^{\prime}$ o estudo de influência deve ser feito via (3.32) e (3.31) substituindo $\ddot{\mathbf{L}}$ por $\ddot{\mathbf{L}}_{E}$ (3.29) e $\Delta$ por $\Delta_{E}$.

\subsubsection{Perturbação das observações}

Nesta seção as observações $\mathbf{Y}_{i}=\left(y_{i 0}, y_{i 1}, \ldots, y_{i p}\right)^{\prime}$ são modificadas por um vetor $\boldsymbol{\omega}_{i}=\left(\omega_{i 0}, \omega_{i 1}, \ldots, \omega_{i p}\right)^{\prime}, i=1, \ldots, n$, segundo esquemas aditivo e multiplicativo.

\section{Modelo homoscedástico}

O modelo perturbado é dado por

$$
\mathbf{Y}_{i}(\omega)=\left(\begin{array}{l}
0 \\
\alpha
\end{array}\right)+\left(\begin{array}{l}
1 \\
\beta
\end{array}\right) x_{i}+\epsilon_{i}
$$

$\operatorname{com} \epsilon_{1}, \ldots, \epsilon_{n} \stackrel{\text { iid }}{\sim} \mathrm{N}_{r}\left(\mathbf{0}, \sigma_{\epsilon \epsilon} \mathbf{I}_{r}\right) \mathrm{e}$

$$
\mathbf{Y}_{i}\left(\boldsymbol{\omega}_{i}\right)= \begin{cases}\mathbf{Y}_{i}+\omega_{i}, & \text { perturbações aditivas, } \\ \mathbf{Y}_{i} \odot \boldsymbol{\omega}_{i}, & \text { perturbações multiplicativas }\end{cases}
$$

$i=1, \ldots, n$, onde " $\odot$ " denota o produto de Hadamard (elemento a elemento) (Styan, 1973). Os vetores $\omega_{0}=0$ ( $n r$-dimensional $)$ e $\omega_{0}=\mathbf{1}_{n r}(q=n r)$, respectivamente, correspondem aos modelos sem perturbação. 
As funções log-verossimilhanças perturbadas $\left(\mathcal{L}^{*}\right)$ dos modelos funcional e estrutural seguem de (3.7) e (3.10) com $\mathbf{Y}_{i}\left(\boldsymbol{\omega}_{i}\right)$ no lugar de $\mathbf{Y}_{i}, i=1, \ldots, n$. Sejam $\Delta_{F}^{\mathrm{a}}$ e $\Delta_{F}^{\mathrm{m}}$ as matrizes de derivadas de segunda ordem de $\mathcal{L}^{*}$ no modelo funcional em relação a $\theta$ e $\omega^{\prime}$ nos esquemas aditivo (a) e multiplicativo $(\mathrm{m})$ e, para o modelo estrutural, $\Delta_{E}^{\mathrm{a}}$ e $\Delta_{E}^{\mathrm{m}}$ são definidas similarmente. Dispostas em blocos estas matrizes são escritas como

$$
\Delta_{F}^{\mathrm{a}}=\left(\begin{array}{c}
\Delta_{\alpha}^{\mathrm{a}} \\
(p \times n r) \\
\Delta_{\beta}^{\mathrm{a}} \\
(p \times n r) \\
\Delta_{\sigma_{\epsilon \epsilon}}^{\mathrm{a}} \\
(1 \times n r)
\end{array}\right) \text { e } \quad \Delta_{E}^{\mathrm{a}}=\left(\begin{array}{c}
\Delta_{\alpha}^{\mathrm{a}} \\
\Delta_{\beta}^{\mathrm{a}} \\
\Delta_{\sigma_{\epsilon \epsilon}}^{\mathrm{a}} \\
\Delta_{\mu_{x}}^{\mathrm{a}} \\
(1 \times n r) \\
\Delta_{\sigma_{x x}}^{\mathrm{a}} \\
(1 \times n r)
\end{array}\right)
$$

onde, por exemplo, $\Delta_{\alpha}^{\mathrm{a}}=\left(\Delta_{\alpha 1}^{\mathrm{a}}, \ldots, \Delta_{\alpha n}^{\mathrm{a}}\right)$, lembrando que $r=p+1$. Extenso algebrismo fornece

$$
\begin{gathered}
\boldsymbol{\Delta}_{\alpha i}^{\mathrm{a}}=\frac{1}{\sigma_{\epsilon \epsilon}}\left(\left(\mathbf{0}, \mathbf{I}_{p}\right)-\frac{1}{c} \boldsymbol{\beta}\left(1, \boldsymbol{\beta}^{\prime}\right)\right) \\
\boldsymbol{\Delta}_{\beta i}^{\mathrm{a}}=\frac{1}{\sigma_{\epsilon \epsilon}}\left(x_{i}\left[\left(\mathbf{0}, \mathbf{I}_{p}\right)-\frac{1}{c} \boldsymbol{\beta}\left(1, \boldsymbol{\beta}^{\prime}\right)\right]+\frac{1}{c}\left[\left(\mathbf{Y}_{1 i}-\overline{\mathbf{Y}_{1}}\right)-\left(x_{i}-\overline{y_{0}}\right) \boldsymbol{\beta}\right]\left(1, \boldsymbol{\beta}^{\prime}\right)\right)
\end{gathered}
$$

$\mathrm{e}$

$$
\Delta_{\sigma_{\epsilon \epsilon} i}^{\mathrm{a}}=\frac{1}{\sigma_{\epsilon \epsilon}^{2}}\left(\mathbf{Y}_{i}-\overline{\mathbf{Y}}-\left(x_{i}-\overline{y_{0}}\right)\left(\begin{array}{c}
1 \\
\beta
\end{array}\right)\right)^{\prime}
$$

no modelo funcional, enquanto no modelo estrutural temos

$$
\begin{gathered}
\Delta_{\alpha i}^{\mathrm{a}}=\frac{1}{\sigma_{\epsilon \epsilon}}\left(\left(\mathbf{0}, \mathbf{I}_{p}\right)-h \boldsymbol{\beta}\left(1, \boldsymbol{\beta}^{\prime}\right)\right) \\
\Delta_{\beta i}^{\mathrm{a}}=\frac{1}{\sigma_{\epsilon \epsilon}}\left(\overline{y_{0}}\left[\left(\mathbf{0}, \mathbf{I}_{p}\right)-h \boldsymbol{\beta}\left(1, \boldsymbol{\beta}^{\prime}\right)\right]+h q_{2 i}\left[\left(\mathbf{0}, \mathbf{I}_{p}\right)-2 h \boldsymbol{\beta}\left(1, \boldsymbol{\beta}^{\prime}\right)\right]+h\left(\mathbf{Y}_{1 i}-\overline{\mathbf{Y}_{1}}\right)\left(1, \boldsymbol{\beta}^{\prime}\right)\right), \\
\Delta_{\sigma_{\epsilon \epsilon}}^{\mathrm{a}}=\frac{1}{\sigma_{\epsilon \epsilon}}\left(\frac{\partial h}{\partial \sigma_{\epsilon \epsilon}} q_{2 i}\left(1, \boldsymbol{\beta}^{\prime}\right)+\frac{1}{\sigma_{\epsilon \epsilon}}\left[\left(\mathbf{Y}_{i}-\overline{\mathbf{Y}}\right)^{\prime}-h q_{2 i}\left(1, \boldsymbol{\beta}^{\prime}\right)\right]\right) \\
\Delta_{\mu_{x i}}^{\mathrm{a}}=\frac{1-h c}{\sigma_{\epsilon \epsilon}}\left(1, \boldsymbol{\beta}^{\prime}\right)
\end{gathered}
$$

$\mathrm{e}$

$$
\Delta_{\sigma_{x x i}}^{\mathrm{a}}=\frac{1}{\sigma_{\epsilon \epsilon}} \frac{\partial h}{\partial \sigma_{x x}}\left(1, \boldsymbol{\beta}^{\prime}\right)
$$


$i=1, \ldots, n$, onde

$$
\frac{\partial h}{\partial \sigma_{\epsilon \epsilon}}=-\frac{h^{2}}{\sigma_{x x}} \quad \text { e } \frac{\partial h}{\partial \sigma_{x x}}=\frac{h(1-h c)}{\sigma_{x x}},
$$

$\mathbf{Y}_{1 i}, \overline{\mathbf{Y}_{1}}$ e $\overline{y_{0}}, x_{i}, c$ e $h$, e $q_{2 i}$ são trazidos de (3.8), (3.17), (3.13), (3.9) e (3.11). Para perturbações multiplicativas basta pós-multiplicar cada matriz obtida por $\mathbf{D}\left(\mathbf{Y}_{i}\right)$. Exemplificando,

$$
\Delta_{\beta i}^{\mathrm{m}}=\Delta_{\beta i}^{\mathrm{a}} \mathrm{D}\left(\mathbf{Y}_{i}\right)
$$

$i=1, \ldots, n$.

Se quisermos modificar somente as medições efetuadas com o $j$-ésimo instrumento, $j=0,1, \ldots, p$, temos

$$
\mathbf{Y}_{i}\left(\omega_{i}\right)=\left(y_{i 0}, \ldots, y_{i j}\left(\omega_{i}\right), \ldots, y_{i p}\right)^{\prime}
$$

onde

$$
y_{i j}\left(\omega_{i}\right)= \begin{cases}y_{i j}+\omega_{i}, & \text { perturbações aditivas, } \\ y_{i j} \omega_{i}, & \text { perturbações multiplicativas, }\end{cases}
$$

$i=1, \ldots, n$, notando que $\omega_{0}=0$ ( $n$-dimensional) e $\omega_{0}=1_{n}(q=n)$ significam nenhuma perturbação. As matrizes $\Delta$ obtidas anteriormente $\left(\Delta_{\alpha i}^{\mathrm{a}}, \Delta_{\alpha i}^{\mathrm{m}}, \ldots\right)$ podem ser aproveitadas aqui, bastando multiplicá-las à direita por $\mathbf{e}_{j}=(0, \ldots, 1, \ldots, 0)^{\prime}$, o $j$-ésimo vetor unitário de $\mathbb{R}^{r}$.

\section{Modelo estrutural heteroscedástico}

Na parametrização de fatores da Seção 3.3 o logaritmo da função verossimilhança perturbada $\left(\mathcal{L}^{*}\right)$ vem de (3.25) com $\mathbf{Y}_{i}\left(\boldsymbol{\omega}_{i}\right)$ calculado em (3.35) no lugar de $\mathbf{Y}_{i}$, $i=1, \ldots, n$. Calculando as derivadas de $\mathcal{L}$ em relação a $\gamma$ e $\omega^{\prime}$ no ponto $\omega=\omega_{0}$ temos as matrizes $\Delta_{E \gamma}^{\mathrm{a}}$ e $\Delta_{E \gamma}^{\mathrm{m}}$ relativas aos esquemas perturbadores aditivo $\left(\omega_{0}=0\right)$ e multiplicativo $\left(\omega_{0}=1_{n r}\right)$, sendo que

$$
\Delta_{E \gamma}^{\mathrm{a}}=\left(\begin{array}{c}
\Delta_{\mu}^{\mathrm{a}} \\
(r \times n r) \\
\Delta_{\lambda}^{\mathrm{a}} \\
(r \times n r) \\
\Delta_{\sigma_{c e}}^{\mathrm{a}} \\
r r \times n r)
\end{array}\right),
$$

$\operatorname{com} \Delta_{\mu}^{\mathrm{a}}=\left(\Delta_{\mu 1}^{\mathrm{a}}, \ldots, \Delta_{\mu n}^{\mathrm{a}}\right) ; \Delta_{\lambda}^{\mathrm{a}}$ e $\Delta_{\sigma_{\epsilon \epsilon}}^{\mathrm{a}}$ são similares. Precisamente,

$$
\Delta_{\mu i}^{\mathrm{a}}=\mathrm{V}^{-1},
$$




$$
\Delta_{\lambda i}^{\mathrm{a}}=c_{*}^{-1}\left[2\left(\mathrm{~W}_{i}^{\prime} \mathbf{D}^{-1}\left(\sigma_{\epsilon \epsilon}\right) \lambda\right) \mathrm{V}^{-1}+\mathbf{D}^{-1}\left(\boldsymbol{\sigma}_{\epsilon \epsilon}\right) \mathrm{W}_{i} \lambda^{\prime} \mathbf{D}^{-1}\left(\boldsymbol{\sigma}_{\epsilon \epsilon}\right)-\left(\mathbf{W}_{i}^{\prime} \mathbf{D}^{-1}\left(\sigma_{\epsilon \epsilon}\right) \boldsymbol{\lambda}\right) \mathbf{D}^{-1}\left(\sigma_{\epsilon \epsilon}\right)\right]
$$

e

$$
\begin{aligned}
\Delta_{\sigma_{\epsilon \epsilon} i}^{\mathrm{a}}= & \mathbf{D}^{-1}\left(\boldsymbol{\sigma}_{\epsilon \epsilon}\right) \mathbf{D}\left(\mathbf{W}_{i}\right) \mathbf{V}^{-1}+c_{*}^{-2} \mathbf{D}^{-2}\left(\sigma_{\epsilon \epsilon}\right)\left(\mathbf{D}(\boldsymbol{\lambda}) \lambda \mathbf{W}_{i}^{\prime} \mathbf{M}\right) \\
& -c_{*}^{-1} \mathbf{W}_{i}^{\prime} \mathbf{D}^{-1}\left(\sigma_{\epsilon \epsilon}\right) \lambda \mathbf{D}^{-2}\left(\sigma_{\epsilon \epsilon}\right) \mathbf{D}(\boldsymbol{\lambda})
\end{aligned}
$$

$i=1, \ldots, n$, onde $\mathbf{V}^{-1}, c_{*}^{-1}, \mathbf{M}$ e $\mathbf{W}_{i}^{\prime}$ se encontram em (3.26), (3.24), (3.28) e (3.34).

A matriz

$$
\Delta_{E \gamma}^{\mathrm{m}}=\left(\begin{array}{c}
\Delta_{\mu}^{\mathrm{m}} \\
\Delta_{\lambda}^{\mathrm{m}} \\
\Delta_{\sigma_{\epsilon \epsilon}}^{\mathrm{m}}
\end{array}\right)
$$

referente ao esquema de perturbação multiplicativo decorre de (3.37) multiplicando à direita cada bloco $\Delta_{\mu i}^{\mathrm{a}}, \Delta_{\lambda i}^{\mathrm{a}}$ e $\Delta_{\sigma_{\epsilon \epsilon} i}^{\mathrm{a}}$ por $\mathbf{D}\left(\mathbf{Y}_{i}\right), i=1, \ldots, n$.

Alterando apenas observações coletadas com o $j$-ésimo instrumento, $j=0,1, \ldots, p$, resulta no vetor $\mathbf{Y}_{i}\left(\omega_{i}\right)$ dado por $(3.36), i=1, \ldots, n$. As matrizes $\boldsymbol{\Delta}_{E \boldsymbol{\gamma}}$ associadas a estas alterações originam-se de $\Delta_{E \mu i}^{\mathrm{a}}, \Delta_{E \mu i}^{\mathrm{m}}, \Delta_{E \lambda i}^{\mathrm{a}}, \ldots$, por meio de

$$
\Delta_{E \mu i}^{\mathrm{a}} \mathbf{e}_{j}, \Delta_{E \mu i}^{\mathrm{m}} \mathbf{e}_{j}, \Delta_{E \lambda i}^{\mathrm{a}} \mathrm{e}_{j}, \ldots,
$$

$i=1, \ldots, n$, onde $\mathbf{e}_{j}$ é o $j$-ésimo vetor unitário de $\mathbb{R}^{r}$.

Trabalhamos na parametrização de regressão $(\theta)$, de modo que necessitamos das matrizes $\Delta_{E}^{\mathrm{a}}$ e $\Delta_{E}^{\mathrm{m}}$, definidas da mesma forma que $\Delta_{E}$ em (3.33) e calculadas como

$$
\Delta_{E}^{\mathrm{a}}=\mathbf{J}_{\gamma \theta} \Delta_{E \gamma}^{\mathrm{a}} \quad \text { e } \quad \Delta_{E}^{\mathrm{m}}=\mathbf{J}_{\gamma \theta} \Delta_{E \gamma}^{\mathrm{m}}
$$

onde $\mathbf{J}_{\gamma \theta}$ é a matriz jacobiana (3.30). Comentários importantes sobre as duas parametrizações foram feitos na p. 43.

Em artigo recente Kwan \& Fung (1998) analisam o modelo de Análise Fatorial (estrutural e heteroscedástico), contemplando os esquemas de perturbação das seções 3.5.1 e 3.5.2. Todavia, a parametrização de fatores impossibilita separarmos a influência de perturbações sobre as estimativas de MV dos vícios ( $\alpha$ e $\beta$ ).

\subsubsection{Perturbação das variâncias dos erros de medição}

Nas seções 3.5.1 e 3.5.2 iniciamos apresentando modelos em que nas distribuições (3.4) e (3.5) os erros de medição têm matriz de covariâncias $\Sigma_{\epsilon \epsilon}=\sigma_{\epsilon \epsilon} \mathbf{I}_{r}$, denominados homoscedásticos. Até o momento, adotando essa estrutura de erros, estudamos 
modificações pertinentes aos dados em si. Investigamos agora o efeito de pequenas perturbações impostas sobre um componente do modelo propriamente dito. A suposição de homogeneidade das variâncias é posta de lado dando lugar a um modelo heteroscedástico com variâncias

$$
\operatorname{var}\left(\epsilon_{i j}\right)=\frac{\sigma_{\epsilon \epsilon}}{\omega_{j}}, \omega_{j}>0, \quad j=0,1, \ldots, p
$$

e $\omega_{0}=1_{r}(q=r=p+1)$ conduz de volta ao modelo homoscedástico. O modelo perturbado pode ser representado como

$$
\mathbf{Y}_{i}=\left(\begin{array}{c}
0 \\
\alpha
\end{array}\right)+\left(\begin{array}{l}
1 \\
\beta
\end{array}\right) x_{i}+\epsilon_{i}
$$

$\epsilon_{1}, \ldots, \epsilon_{n} \stackrel{\text { iid }}{\sim} \mathrm{N}_{r}\left(0, \sigma_{\epsilon \epsilon} \mathbf{D}^{-1}(\boldsymbol{\omega})\right)$, onde $\boldsymbol{\omega}=\left(\omega_{0}, \omega_{1}, \ldots, \omega_{n}\right)^{\prime}$. No modelo funcional perturbado, dados $\alpha$ e $\beta$, os EMV dos parâmetros incidentais $\dot{x}_{i}$ são

$$
\widehat{x_{i}}(\omega)=\widehat{x}_{i}(\boldsymbol{\alpha}, \boldsymbol{\beta}, \omega)=\frac{1}{c(\omega)}\left[\mathbf{Y}_{i}^{\prime}-\left(0, \boldsymbol{\alpha}^{\prime}\right)\right] \mathbf{D}(\omega)\left(1, \boldsymbol{\beta}^{\prime}\right)^{\prime},
$$

$i=1, \ldots, n$, onde $c(\omega)=\mathbf{b}^{\prime} \mathbf{D}(\omega) \mathbf{b}$, notando que $c\left(\omega_{0}\right)=c$ e $\widehat{x_{i}}\left(\omega_{0}\right)=\widehat{x_{i}}$, vistos em (3.9) e (3.13). O logaritmo da função verossimilhança correspondente é dado por

$$
\mathcal{L}^{*}(\boldsymbol{\theta} ; \boldsymbol{\omega}, \mathbf{Y})=\sum_{i=1}^{n} \omega_{i} l\left(\boldsymbol{\theta} ; \boldsymbol{\omega}, \mathbf{Y}_{i}\right)
$$

onde

$$
\begin{gathered}
l\left(\boldsymbol{\theta} ; \mathbf{Y}_{i}\right)=-\frac{r}{2}+\frac{1}{2} \log |\mathbf{D}(\omega)|-\frac{1}{2} Q_{F}\left(\boldsymbol{\theta} ; \boldsymbol{\omega}, \mathbf{Y}_{i}\right), \\
Q_{F}\left(\boldsymbol{\theta} ; \omega, \mathbf{Y}_{i}\right)=\frac{1}{\sigma_{\epsilon \epsilon}}\left\{\left[\mathbf{Y}_{i}^{\prime}-\left(0, \boldsymbol{\alpha}^{\prime}\right)\right] \mathbf{D}(\omega)\left[\mathbf{Y}_{i}-\left(0, \boldsymbol{\alpha}^{\prime}\right)^{\prime}\right] c(\boldsymbol{\omega}) \widehat{x}_{i}^{2}(\boldsymbol{\omega})\right\}
\end{gathered}
$$

$i=1, \ldots, n$. Calculando as derivadas de segunda ordem de $\mathcal{L}^{*}$ acima em relação a $\theta$ e $\omega^{\prime}$ nos pontos $\hat{\theta}$ e $\omega_{0}$ temos que

$$
\begin{gathered}
\Delta_{\alpha}=0 \\
\Delta_{\beta}=\frac{1}{c \sigma_{\epsilon \epsilon}} \mathbf{B}_{1} \mathrm{D}(b), \\
\Delta_{\sigma_{\epsilon \epsilon}}=\frac{n}{2 \sigma_{\epsilon \epsilon}^{2}}\left[\mathbf{1}_{r}^{\prime} \mathrm{D}(v)-\frac{b^{\prime} \mathrm{S} b}{c^{2}} b^{\prime} \mathrm{D}(b)\right],
\end{gathered}
$$


com

$$
\mathbf{B}_{1}=n\left(\mathbf{S}_{10}-\frac{1}{c}\left(\mathbf{S}_{10}+\mathbf{S}_{11} \beta\right), \mathbf{S}_{11}-\frac{1}{c}\left(\mathbf{S}_{10}+\mathbf{S}_{11} \beta\right) \beta^{\prime}\right)
$$

e $\boldsymbol{v}=\left(S_{00}, S_{11}, \ldots, S_{p p}\right)^{\prime}$ é o vetor dos elementos da diagonal principal de $\mathbf{S}$ (3.15), subdividida em (3.19).

Por outro lado, no modelo estrutural perturbado as observações são tais que

$$
\mathbf{Y}_{1}, \ldots, \mathbf{Y}_{n} \stackrel{\text { iid }}{\sim} \mathrm{N}_{r}\left(\left(\begin{array}{c}
0 \\
\boldsymbol{\alpha}
\end{array}\right)+\left(\begin{array}{l}
1 \\
\beta
\end{array}\right) \mu_{x}, \mathrm{~V}(\omega)\right)
$$

onde $\mathrm{V}(\boldsymbol{\omega})=\sigma_{x x} \boldsymbol{b} \boldsymbol{b}^{\prime}+\sigma_{\epsilon \epsilon} \mathbf{D}^{-1}(\boldsymbol{\omega})$, implicando que

$$
\mathcal{L}^{*}(\boldsymbol{\theta} ; \boldsymbol{\omega}, \mathbf{Y})=\sum_{i=1}^{n} \omega_{i} l\left(\boldsymbol{\theta} ; \boldsymbol{\omega}, \mathbf{Y}_{i}\right)
$$

onde

$$
\begin{aligned}
l\left(\boldsymbol{\theta} ; \boldsymbol{\omega}, \mathbf{Y}_{i}\right)= & -\frac{r}{2} \log (2 \pi)-\frac{1}{2} \log |\boldsymbol{V}(\boldsymbol{\omega})|-\frac{1}{2} Q_{E}\left(\boldsymbol{\theta} ; \boldsymbol{\omega}, \mathbf{Y}_{i}\right) \\
Q_{E}\left(\boldsymbol{\theta} ; \boldsymbol{\omega}, \mathbf{Y}_{i}\right)= & \frac{1}{\sigma_{\epsilon \epsilon}}\left\{\left[\mathbf{Y}_{i}^{\prime}-\left(0, \boldsymbol{\alpha}^{\prime}\right)-\boldsymbol{b}^{\prime} \mu_{x}\right] \mathbf{D}(\boldsymbol{\omega})\left[\mathbf{Y}_{i}-\left(0, \boldsymbol{\alpha}^{\prime}\right)^{\prime}-\boldsymbol{b} \mu_{x}\right]-h(\boldsymbol{\omega})\right. \\
& \left.\times\left[\boldsymbol{b}^{\prime} \mathbf{D}(\boldsymbol{\omega})\left(\mathbf{Y}_{i}-\left(0, \boldsymbol{\alpha}^{\prime}\right)^{\prime}-\boldsymbol{b} \mu_{x}\right)\right]^{2}\right\}
\end{aligned}
$$

com

$$
h(\omega)=\frac{\sigma_{x x}}{\sigma_{\epsilon \epsilon}+\sigma_{x x} c(\omega)},
$$

e $c(\omega)$ como antes, $i=1, \ldots, n$. Derivando $\mathcal{L}^{*}$ acima em relação a $\theta$ e $\boldsymbol{\omega}^{\prime}$ e avaliando estas derivadas em $\boldsymbol{\theta}=\widehat{\boldsymbol{\theta}}$ e $\boldsymbol{\omega}=\omega_{0}$ resulta

$$
\Delta_{\alpha}=0
$$

$$
\begin{gathered}
\boldsymbol{\Delta}_{\beta}=\frac{n h}{\sigma_{\epsilon \epsilon}}\left\{-\left(\sigma_{\epsilon \epsilon}+h \boldsymbol{b}^{\prime} \mathbf{S} \boldsymbol{b}\right)(\mathbf{0}, \mathbf{D}(\boldsymbol{\beta}))+h \sigma_{\epsilon \epsilon} \boldsymbol{\beta} \boldsymbol{b}^{\prime} \mathbf{D}(\boldsymbol{b})+\left(\mathbf{0}, \mathbf{D}\left(\mathbf{S}_{10}+\mathbf{S}_{11} \boldsymbol{\beta}\right)\right)\right. \\
\left.+\left[\left(\mathbf{S}_{10}, \mathbf{S}_{11}\right)-h\left(\mathbf{S}_{10}+\mathbf{S}_{11} \boldsymbol{\beta}\right) \boldsymbol{b}^{\prime}-2 h \boldsymbol{\beta} \boldsymbol{b}^{\prime} \mathbf{S}+2 h^{2}\left(\boldsymbol{b}^{\prime} \mathbf{S} \boldsymbol{b}\right) \boldsymbol{\beta} \boldsymbol{b}^{\prime}\right] \mathbf{D}(\boldsymbol{b})\right\} \\
\boldsymbol{\Delta}_{\sigma_{\epsilon \epsilon}}=h n\left\{\frac{h}{\sigma_{x x}}\left(\frac{1}{2}+\frac{h \boldsymbol{b}^{\prime} \mathbf{S} \boldsymbol{b}}{\sigma_{\epsilon \epsilon}}+\frac{\sigma_{x x} \boldsymbol{b}^{\prime} \mathbf{S} \boldsymbol{b}}{2 \sigma_{\epsilon \epsilon}^{2}}\right) \boldsymbol{b}^{\prime} \mathbf{D}(\boldsymbol{b})\right. \\
\left.+\frac{1}{2 h \sigma_{\epsilon \epsilon}^{2}} \mathbf{1}_{r}^{\prime} \mathbf{D}(\boldsymbol{v})-\frac{1}{\sigma_{\epsilon \epsilon}}\left(\frac{h}{\sigma_{x x}}+\frac{1}{\sigma_{\epsilon \epsilon}}\right) \boldsymbol{b}^{\prime} \mathbf{S D}(\boldsymbol{b})\right\} \\
\boldsymbol{\Delta}_{\mu_{x}}=\mathbf{0}
\end{gathered}
$$


e

$$
\Delta_{\sigma_{x x}}=-\frac{h n(1-h c)}{2 \sigma_{\epsilon \epsilon} \sigma_{x x}}\left\{\left[\sigma_{\epsilon \epsilon}+2 h \boldsymbol{b}^{\prime} \mathbf{S} \boldsymbol{b}\right] \boldsymbol{b}^{\prime} \mathbf{D}(\boldsymbol{b})-2 \boldsymbol{b}^{\prime} \mathbf{S D}(\boldsymbol{b})\right\},
$$

com $\mathbf{D}(\boldsymbol{v})$ proveniente de (3.38).

Agrupando os blocos formamos as matrizes $\Delta_{F}$ e $\Delta_{E}$, relativas aos modelos funcional e estrutural:

$$
\Delta_{F}=\left(\begin{array}{c}
\Delta_{\alpha} \\
(p \times r) \\
\Delta_{\beta} \\
(p \times r) \\
\Delta_{\sigma_{\epsilon \epsilon}} \\
(1 \times r)
\end{array}\right) \quad \text { e } \quad \Delta_{E}=\left(\begin{array}{c}
\Delta_{\alpha} \\
\Delta_{\beta} \\
\Delta_{\sigma_{\epsilon \epsilon}} \\
\Delta_{\mu_{x}} \\
(1 \times r) \\
\Delta_{\sigma_{x x}} \\
(1 \times r)
\end{array}\right)
$$

Se a suposição de homoscedasticidade for plausível, os componentes de $\left|l_{\max }\right|$ devem ter valores similares. Caso contrário, o vetor $\boldsymbol{l}_{\max }$ serve como um guia na construção de um modelo revisado. Na Seção 3.6 retornaremos a este ponto.

\subsection{Aplicações}

Dois conjuntos de dados reais serão analisados. Estamos interessados no conjunto de parâmetros $(\theta)$ e no subconjunto $\theta_{1}=\left(\alpha^{\prime}, \beta^{\prime}\right)^{\prime}$, que representa os vícios aditivos e multiplicativos dos instrumentos. Os modelos funcional e estrutural conduzem às mesmas conclusões nas duas aplicações. Optamos pelo modelo estrutural, pois possibilita uma comparação entre os modelos homoscedástico (Seção 3.2) e heteroscedástico (Seção 3.3).

Adotamos a linguagem de programação Ox (Doornik, 1998). As expressões desenvolvidas nas seções anteriores passaram por verificação numérica utilizando funções de cálculo de derivadas disponíveis na linguagem.

Exemplo 3.1 Dados de Chipkevitch et al. (1996). Medições de volume testicular direito foram feitas em 42 adolescentes usando cinco técnicas diferentes: ultrasom (US), método gráfico proposto pelos autores (I), medição dimensional (II), orquidômetro de Prader (III) e orquidômetro de anel (IV), sendo que ultra-som é o método de referência. As medições encontram-se na Tabela 3.1. Uma transformação por raiz cúbica melhora a aproximação pela distribuição normal multivariada. A 


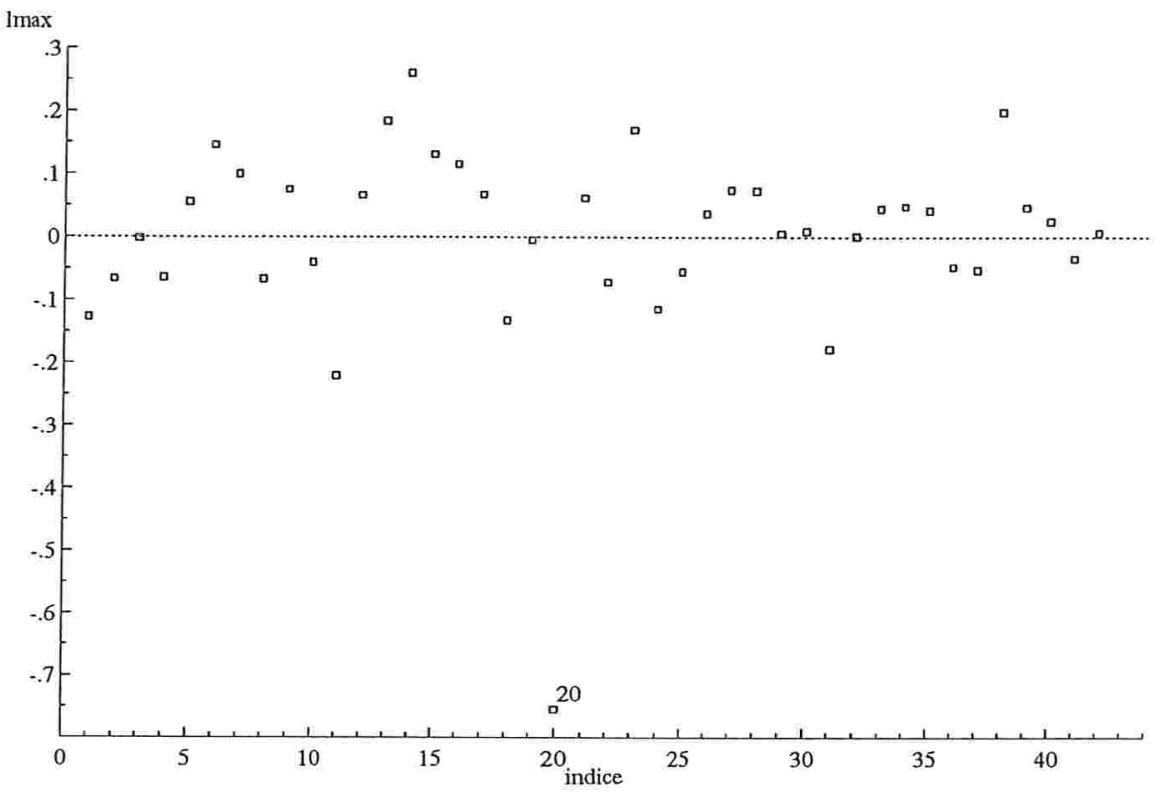

Figura 3.2: Gráfico de índices de $\boldsymbol{l}_{\max }$ na perturbação da ponderação de casos Exemplo 3.1.

suposição de homoscedasticidade (Seção 3.5.3) parece razoável se o interesse recai em $\theta$, pois

$$
\left|l_{\max }\right|=(0.43504,0.51309,0.43625,0.42440,0.42078)^{\prime} .
$$

As estimativas de MV dos parâmetros são $\widehat{\alpha_{1}}=0.068428, \widehat{\alpha_{2}}=0.030502, \widehat{\alpha_{3}}=$ $0.033242, \widehat{\alpha_{4}}=0.38755, \widehat{\beta_{1}}=0.93198, \widehat{\beta_{2}}=0.96856, \widehat{\beta_{3}}=1.0319, \widehat{\beta_{4}}=0.89725$, $\widehat{\sigma_{\epsilon \epsilon}}=0.0054155, \widehat{\mu_{x}}=2.1005$ e $\widehat{\sigma_{x x}}=0.12333$.

No esquema de perturbação da ponderação dos casos (Seção 3.5.1) temos $C_{l_{\max }}=$ 4.582 e o adolescente 20 se destaca, como se vê na Figura 3.2. Seguindo a abordagem de Wu \& Luo (1993) apresentamos na Figura 3.3 gráficos do afastamento da verossimilhança $L D(\omega(a))$ versus $a$ para as direções $\boldsymbol{l}_{\max }$ e $e_{20}$, onde $\omega(a)=1_{42}+a l_{\max }$ e $\omega(a)=1_{42}-a e_{20}$, respectivamente, com $\boldsymbol{e}_{20}$ denotando o vigésimo vetor unitário em $\mathbb{R}^{42}$. As curvas têm comportamento similar e para $a>0$ têm grande variação, indicando que o adolescente 20 é influente (em sentido global).

Em seguida examinamos os efeitos das perturbações das medições tomadas com as cinco técnicas (Seção 3.5.2), uma técnica de cada vez. Os valores de $C_{l_{\max }}$ para 
Tabela 3.1: Volume testicular (em ml) - Exemplo 3.1.

\begin{tabular}{cccccc}
\hline Número do & \multicolumn{5}{c}{ Métodos } \\
\cline { 2 - 6 } adolescente & US & I & II & III & IV \\
\hline 1 & 5.0 & 7.5 & 5.9 & 8.0 & 9.0 \\
2 & 5.7 & 5.0 & 4.8 & 6.0 & 10.0 \\
3 & 7.4 & 5.0 & 6.8 & 9.0 & 12.0 \\
4 & 2.6 & 3.5 & 3.1 & 4.0 & 4.0 \\
5 & 5.7 & 5.0 & 5.0 & 6.0 & 7.0 \\
6 & 6.1 & 5.0 & 4.4 & 7.0 & 8.0 \\
7 & 6.2 & 5.0 & 6.0 & 8.0 & 9.0 \\
8 & 10.4 & 10.0 & 8.8 & 10.0 & 10.0 \\
9 & 9.1 & 7.5 & 7.9 & 10.0 & 11.0 \\
10 & 14.8 & 10.0 & 13.0 & 12.0 & 15.0 \\
11 & 16.4 & 12.5 & 10.3 & 17.5 & 17.5 \\
12 & 9.6 & 7.5 & 8.2 & 10.0 & 11.0 \\
13 & 15.7 & 15.0 & 19.8 & 20.0 & 20.0 \\
14 & 3.0 & 2.0 & 2.0 & 3.0 & 4.0 \\
15 & 16.4 & 15.0 & 17.3 & 20.0 & 20.0 \\
16 & 17.6 & 15.0 & 17.3 & 20.0 & 22.5 \\
17 & 10.0 & 7.5 & 7.9 & 12.0 & 12.0 \\
18 & 4.1 & 3.5 & 4.4 & 4.0 & 6.0 \\
19 & 12.7 & 10.0 & 11.4 & 12.0 & 12.0 \\
20 & 2.7 & 3.5 & 4.1 & 2.5 & 6.0 \\
21 & 10.2 & 10.0 & 11.1 & 12.0 & 13.5 \\
22 & 16.5 & 10.0 & 15.3 & 15.0 & 15.0 \\
23 & 4.5 & 3.5 & 3.9 & 6.0 & 7.0 \\
24 & 5.6 & 5.0 & 4.5 & 4.5 & 6.0 \\
25 & 11.0 & 7.5 & 9.7 & 9.0 & 11.0 \\
26 & 9.2 & 10.0 & 11.3 & 12.0 & 13.5 \\
27 & 8.5 & 7.5 & 8.8 & 12.0 & 12.0 \\
28 & 5.4 & 5.0 & 6.1 & 8.0 & 8.0 \\
29 & 6.7 & 7.5 & 7.2 & 10.0 & 8.0 \\
30 & 5.3 & 5.0 & 5.9 & 8.0 & 10.0 \\
31 & 20.0 & 20.0 & 16.3 & 25.0 & 22.5 \\
32 & 18.8 & 15.0 & 16.3 & 20.0 & 25.0 \\
33 & 13.9 & 12.5 & 12.2 & 15.0 & 17.5 \\
34 & 9.4 & 10.0 & 10.3 & 12.0 & 13.5 \\
35 & 9.1 & 7.5 & 10.8 & 12.0 & 12.0 \\
36 & 14.1 & 15.0 & 13.0 & 13.5 & 15.0 \\
37 & 9.3 & 10.0 & 8.4 & 10.0 & 10.0 \\
38 & 20.9 & 20.0 & 22.1 & 25.0 & 25.0 \\
39 & 11.5 & 10.0 & 10.6 & 15.0 & 13.5 \\
40 & 9.7 & 10.0 & 9.7 & 11.0 & 12.0 \\
41 & 13.7 & 12.5 & 11.6 & 17.5 & 15.0 \\
42 & 8.9 & 10.0 & 8.1 & 12.0 & 12.0 \\
\hline & & & & &
\end{tabular}




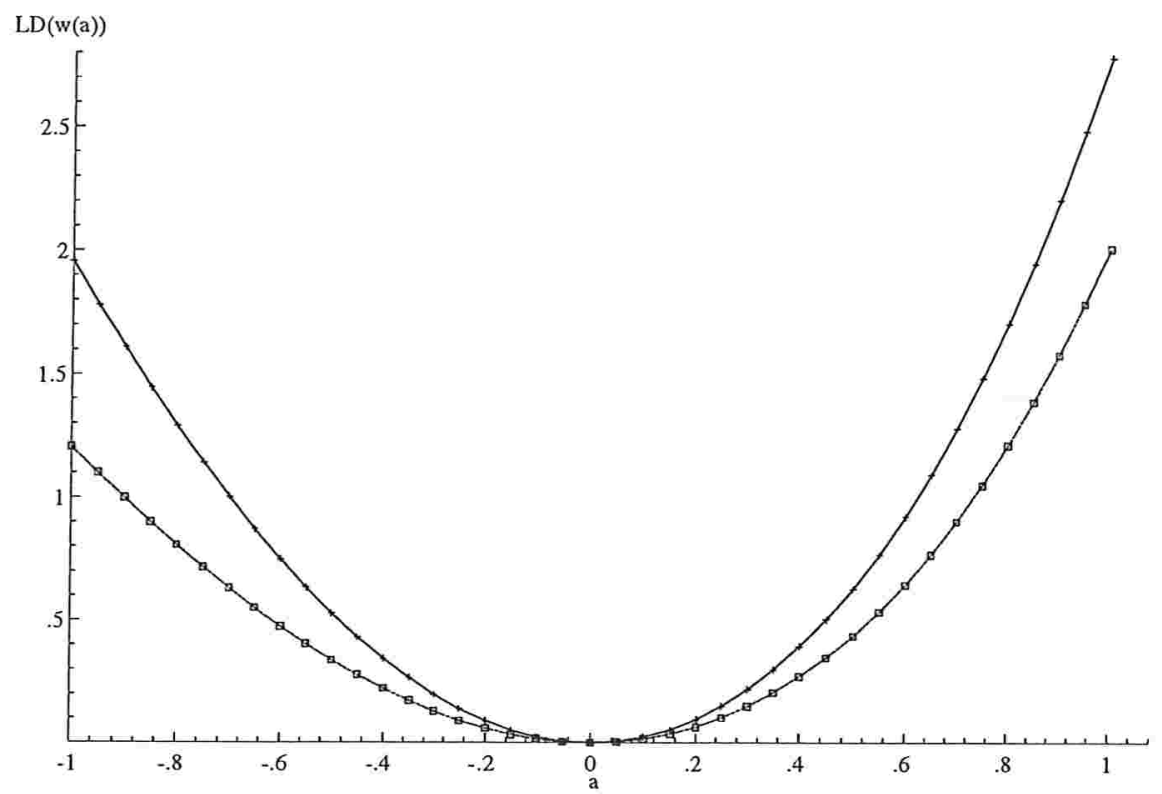

Figura 3.3: Gráficos do afastamento da verossimilhança $L D(\omega(a))$ versus a nas direções $l_{\max }(+)$ e $e_{20}(\square)$ - Exemplo 3.1 .

perturbações aditivas são 235.14 (US), 243.83 (I), 238.8 (II), 231.20 (III) e 246.50 (IV), enquanto para perturbações multiplicativas os valores de $C_{l_{\max }}$ são 1412.6 (US), 1367.6 (I), 1400.1 (II), 1515.2 (III) e 1680.9 (IV), revelando que o afastamento da verossimilhança é mais sensível a perturbações multiplicativas. Além disso, o afastamento da verossimilhança é mais sensível a perturbações nas medições do que a perturbações na ponderação dos casos (vide o valor de $C_{l_{\max }}$ no parágrafo anterior). As Figuras 3.4 a 3.6 ilustram as diferenças entre as perturbações aditivas e multiplicativas. A técnica IV é escolhida devido aos maiores valores de $C_{l_{\max }}$. Notamos que na maioria dos adolescentes o maior volume testicular é fornecido pela técnica IV.

Adotando o modelo estrutural heteroscedástico (Seção 3.3), no esquema de perturbação da ponderação de casos o gráfico de índices de $\left|l_{\max }\right|$ (não exibido) se assemelha à Figura 3.2, reforçando a indicação de homogeneidade das variâncias sugerida pelo vetor $\boldsymbol{l}_{\max }$ em (3.39). Sendo assim, optaríamos pelo modelo homoscedástico (mais simples).

Exemplo 3.2 Dados de Barnett (1969). Dois instrumentos são empregados para medir a capacidade vital do pulmão humano por operadores experientes e inexperientes em um grupo de 72 pacientes. As combinações de instrumentos e níveis 


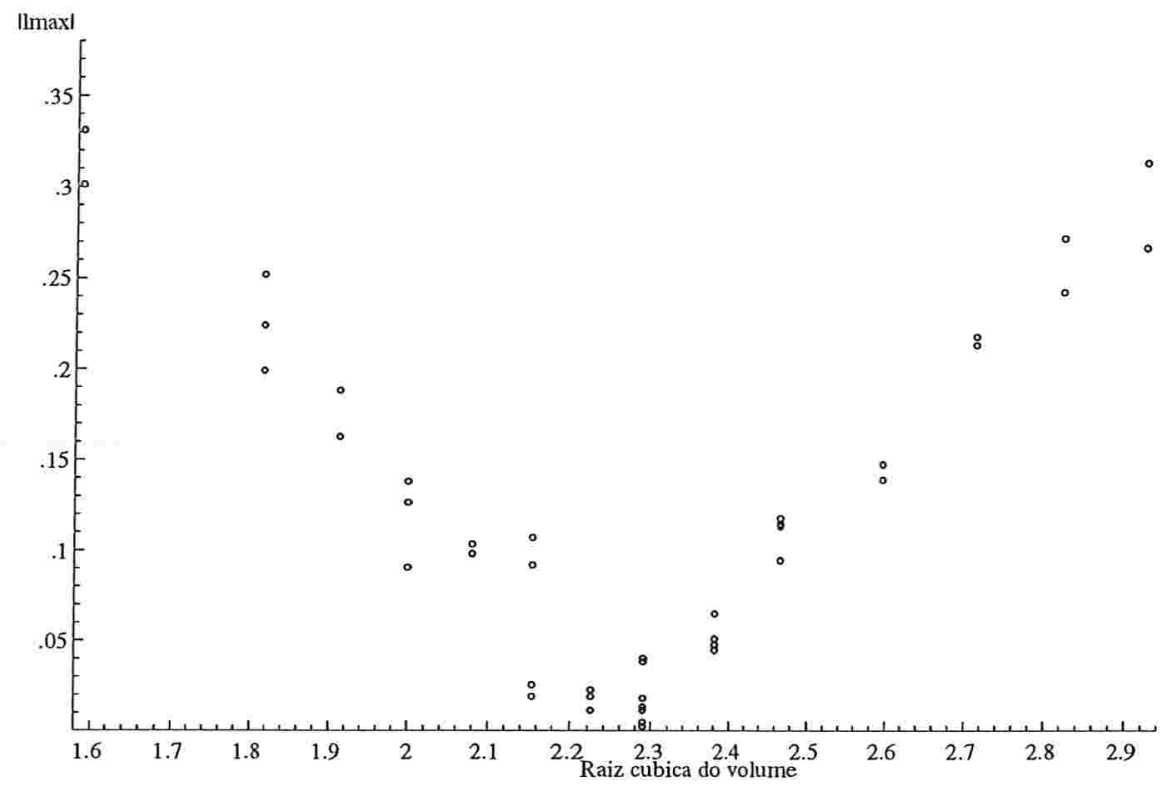

Figura 3.4: Gráfico de $\left|\boldsymbol{l}_{\max }\right|$ versus a raiz cúbica das medições efetuadas com a técnica IV, perturbações aditivas - Exemplo 3.1.

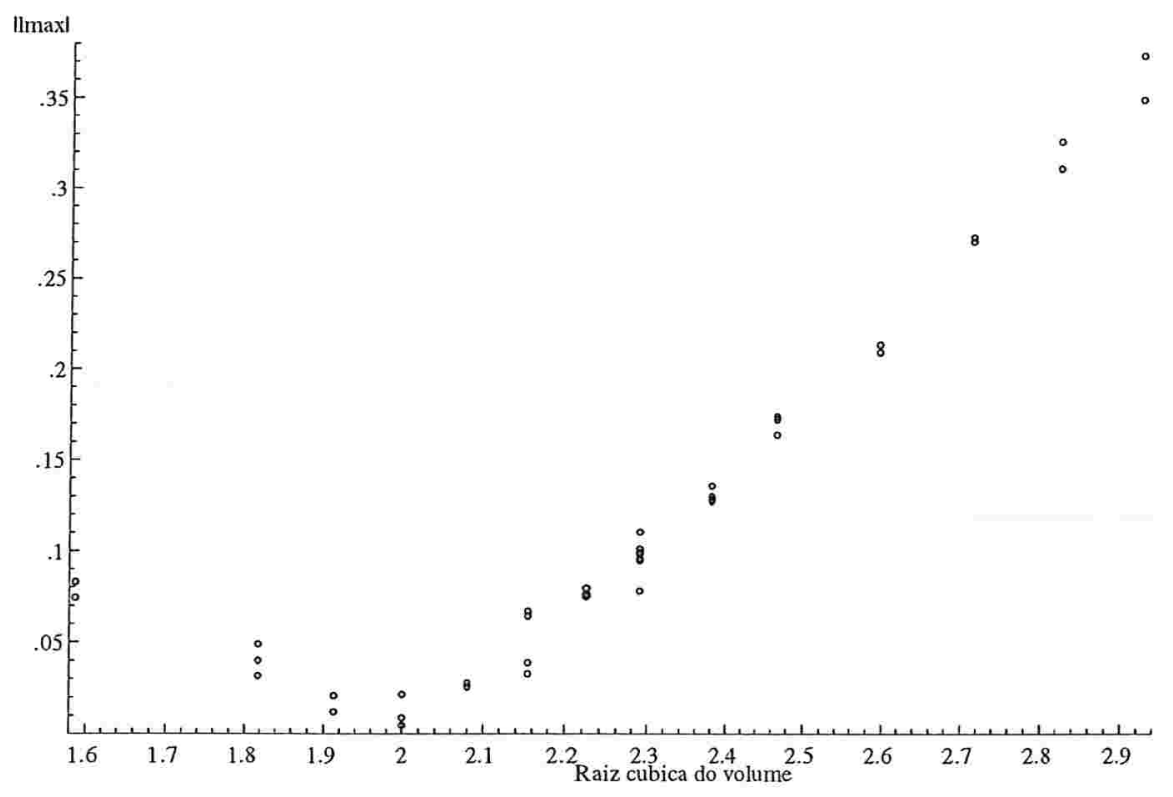

Figura 3.5: Gráfico de $\left|l_{\max }\right|$ versus a raiz cúbica das medições efetuadas com a técnica IV, perturbações multiplicativas - Exemplo 3.1 . 


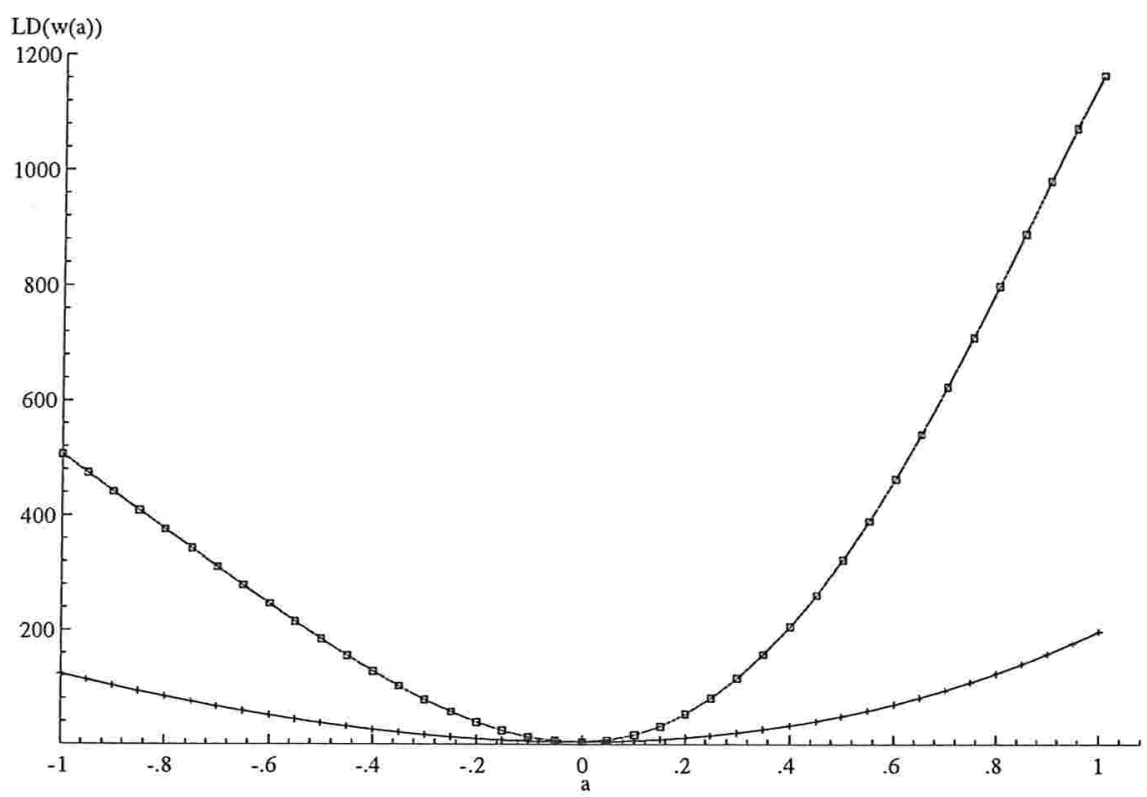

Figura 3.6: Gráficos do afastamento da verossimilhança $L D(\omega(a))$ versus a nas direções $l_{\max }$ impondo perturbações aditivas $(+)$ e multiplicativas $(\square)$ nas medições feitas com a técnica IV - Exemplo 3.1.

de experiência dos operadores geram quatro maneiras de se tomarem as medições. Trabalhando com o modelo estrutural homoscedástico (Seção 3.2), de acordo com o esquema de perturbação das variâncias (Seção 3.5.3) temos $C_{l_{\max }}(\theta)=59.35$,

$$
l_{\max }=(0.70839,0.30780,0.38823,0.50271)^{\prime} \text {, }
$$

$C_{l_{\max }}\left(\theta_{1}\right)=1.221 \mathrm{e}$

$$
l_{\max }=(-0.73253,-0.17966,0.36928,0.54291)^{\prime} .
$$

Os vetores $\left|l_{\max }\right|$ sinalizam heterogeneidade das variâncias, quer estejamos interessados em $\theta$ ou $\theta_{1}$. Nosso interesse específico está em $\theta_{1}$. O vetor $l_{\max }$ é, então, usado para ajustar as variâncias dos erros de medição através de

$$
\operatorname{var}\left(\epsilon_{i j}\right)=\frac{\sigma_{\epsilon \epsilon}}{1+a l_{\max _{j}}}, j=0,1, \ldots, p
$$

Transformamos os dados $\mathbf{Y}_{i}$ resultando em

$$
\left(\left(1+a l_{\text {max }_{0}}\right)^{1 / 2} y_{i 0},\left(1+a l_{\text {max }_{1}}\right)^{1 / 2} y_{i 1}, \ldots,\left(1+a l_{\text {max }_{p}}\right)^{1 / 2} y_{i p}\right)^{\prime},
$$




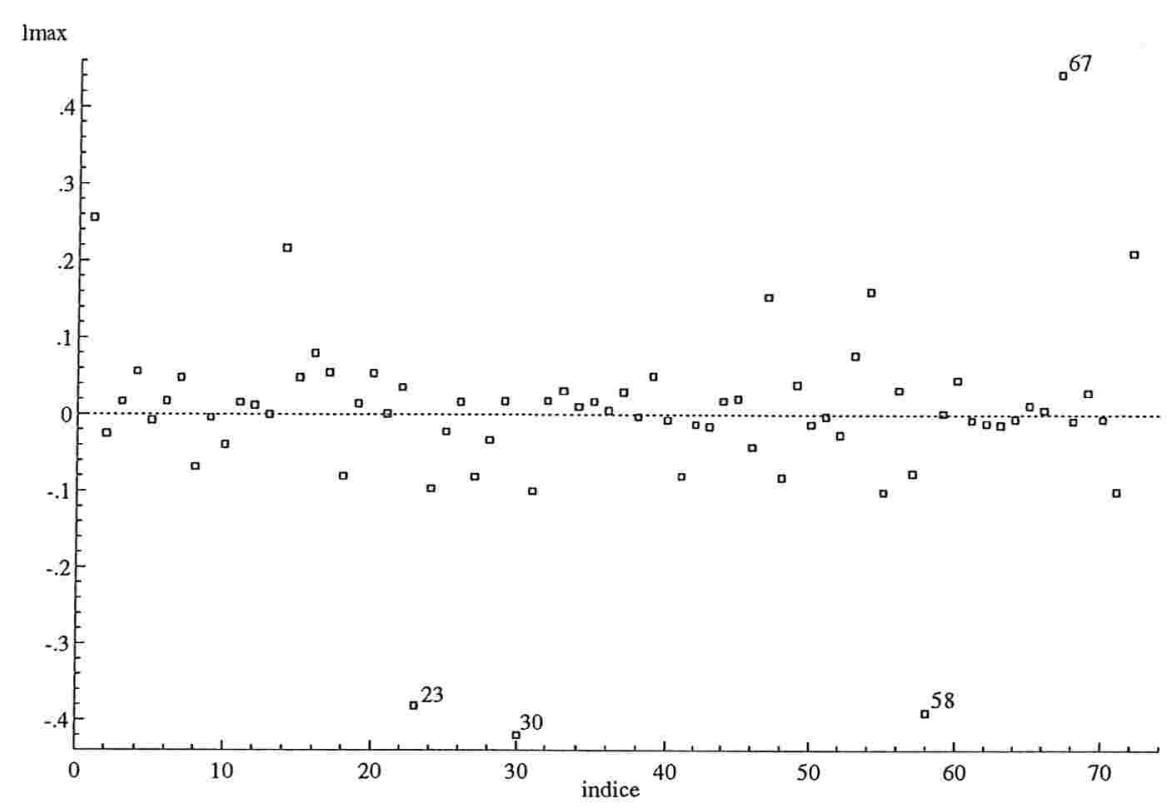

Figura 3.7: Gráfico de índices de $l_{\max }$ na perturbação da ponderação de casos Exemplo 3.2.

$i=1, \ldots, n$, acarretando um novo $\boldsymbol{l}_{\max }\left(\boldsymbol{l}_{\max }^{*}\right.$, digamos $)$. Lançando em um gráfico

$$
\delta_{a}=\max _{j=0,1, \ldots, p}\left|l_{\max _{j}}^{*}\right|-\min _{j=0,1, \ldots, p}\left|l_{\max _{j}}^{*}\right|
$$

versus $a$ selecionamos o valor de $a$ que corresponde ao mínimo de $\delta_{a}$. Partindo de $l_{\max }$ em (3.40) com $\delta_{a}=0.55287$ obtemos $a=0.2295$ e $\delta_{a}=0.36783$. Nosso modelo de trabalho passa a ter variâncias

$$
\frac{\sigma_{\epsilon \epsilon}}{0.83188}, \frac{\sigma_{\epsilon \epsilon}}{0.95877}, \frac{\sigma_{\epsilon \epsilon}}{1.0848} \text { e } \frac{\sigma_{\epsilon \epsilon}}{1.1246} .
$$

As estimativas dos parâmetros são $\widehat{\alpha_{1}}=-190.95, \widehat{\alpha_{2}}=-528.78, \widehat{\alpha_{3}}=-447.39$, $\widehat{\beta_{1}}=1.0537, \widehat{\beta_{2}}=1.1920, \widehat{\beta_{3}}=1.1351, \widehat{\sigma_{\epsilon \epsilon}}=33964, \widehat{\mu_{x}}=2246.1 \mathrm{e} \widehat{\sigma_{x x}}=534031$. Perturbando as ponderações dos casos (Seção 3.5.1) vemos em destaque os casos 23, 30, 58 e 67, como podemos concluir da Figura 3.7. Na seqüência comparamos influência local e eliminação de casos. Seguindo Zhao \& Lee (1998), a distância de Cook pode ser definida por

$$
D 2_{i}=\left(\widehat{\boldsymbol{\theta}}_{1(i)}-\widehat{\boldsymbol{\theta}}_{1}\right)^{\prime}\left[\mathbf{V}\left(\widehat{\boldsymbol{\theta}}_{1}\right)\right]^{-1}\left(\widehat{\boldsymbol{\theta}}_{1(i)}-\widehat{\boldsymbol{\theta}}_{1}\right) / 2 p,
$$




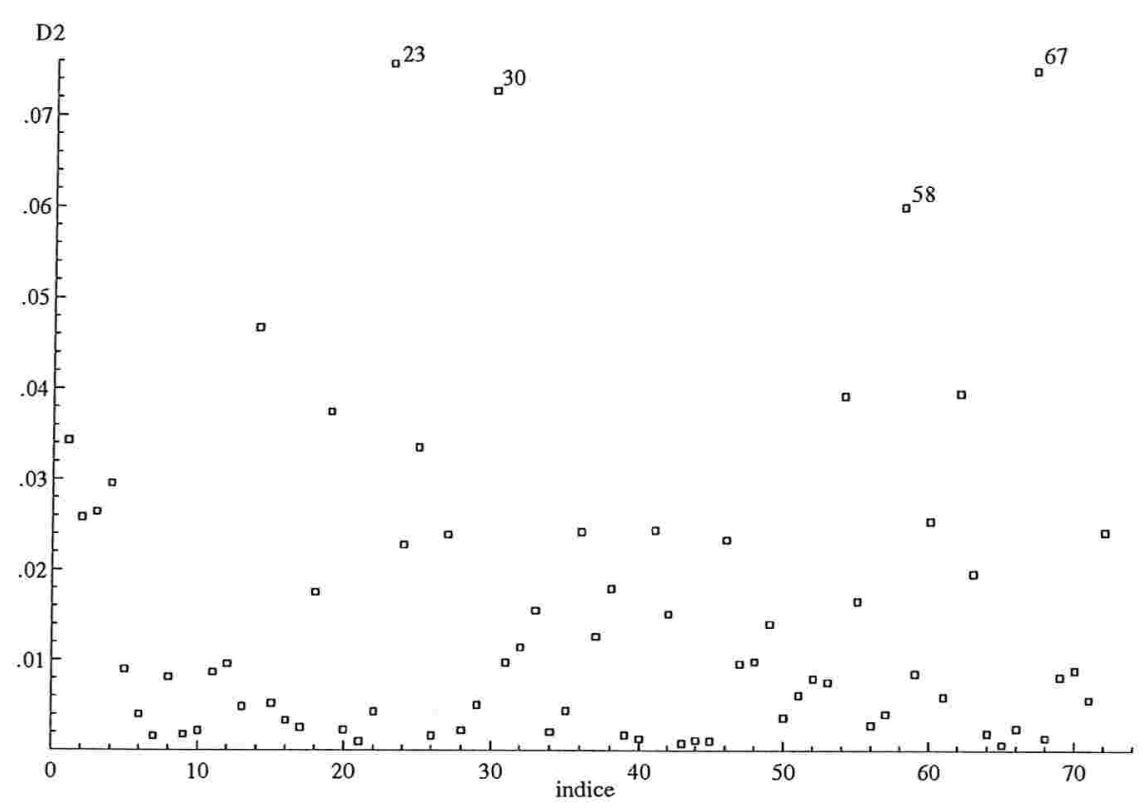

Figura 3.8: Gráfico de índices da distância de Cook $\left(D 2_{i}\right)$ - Exemplo 3.2 .

$i=1, \ldots, n$, onde $\widehat{\theta}_{1(i)}$ denota as estimativas dos parâmetros eliminando-se o caso $i$, e $\mathrm{V}\left(\widehat{\boldsymbol{\theta}}_{1}\right)$ estima consistentemente $\operatorname{var}\left(\widehat{\boldsymbol{\theta}}_{1}\right)$. Tal estimativa pode ser calculada de $\left(-\ddot{\mathrm{L}}_{E}\right)^{-1}$ em (3.20). A Figura 3.8 traz o gráfico de índices de $D 2_{i}, i=1, \ldots, n$. Mais uma vez os casos 23, 30, 58 e 67 se destacam. Gráficos do afastamento da verossimilhança $L D_{1}(\omega(a))$ versus $a$ nas direções $l_{\max }$ e $e_{67}$ compõem a Figura 3.9. A curva $L D_{1}$ na direção $e_{67}$ tem variação bem menor que a curva $L D_{1}$ na direção $l_{\max }$ (comparamos com $e_{67}$ porque na Figura 3.7 o caso 67 tem o maior componente em $\left|l_{\max }\right|$ ). Como o vetor $l_{\max }$ é dominado pelos casos $23,30,58$ e 67, esses resultados indicam influência global conjunta. No Exemplo 3.1 a Figura 3.3 representa a situação de influência global de um único caso.

Em vez de ajustarmos as variâncias dos erros de medição, invocamos diretamente o modelo estrutural heteroscedástico (Seção 3.3). As estimativas de MV dos parâmetros são $\widehat{\alpha_{1}}=-204.46, \widehat{\alpha_{2}}=-528.58, \widehat{\alpha_{3}}=-437.25, \widehat{\beta_{1}}=1.0597$, $\widehat{\beta_{2}}=1.1919, \widehat{\beta_{3}}=1.1306, \widehat{\sigma_{\epsilon \epsilon 0}}=50248, \widehat{\sigma_{\epsilon \epsilon 1}}=19151, \widehat{\sigma_{\epsilon \epsilon 2}}=29236, \widehat{\sigma_{\epsilon \epsilon 3}}=38843$, $\widehat{\mu_{x}}=2246.1$ e $\widehat{\sigma_{x x}}=534087$. Perturbando as ponderações dos casos (Seção 3.5.1) e traçando o gráfico de índices de $\boldsymbol{l}_{\max }$ (Figura 3.10) concluímos que o caso 54 se junta aos casos 23, 30, 58 e 67 já presentes na Figura 3.7. Sendo assim, o vetor $\boldsymbol{l}_{\max }$ parece ser um elemento útil na detecção de heterogeneidade das variâncias dos 


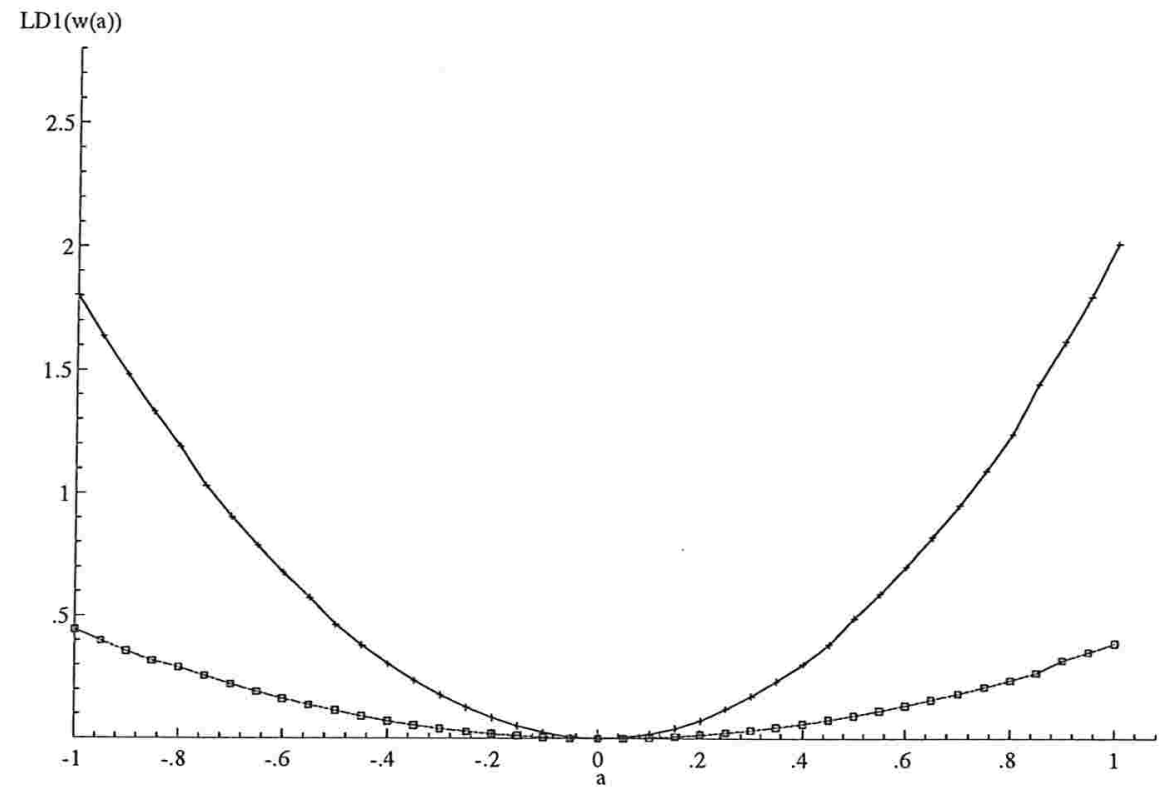

Figura 3.9: Gráficos do afastamento da verossimilhança $L D_{1}(\omega(a))$ versus a nas direções $l_{\max }(+)$ e $e_{67}(\square)$ - Exemplo 3.2.

erros de medição, mas incapaz de ser usado para ajustar as variâncias no modelo homoscedástico. 

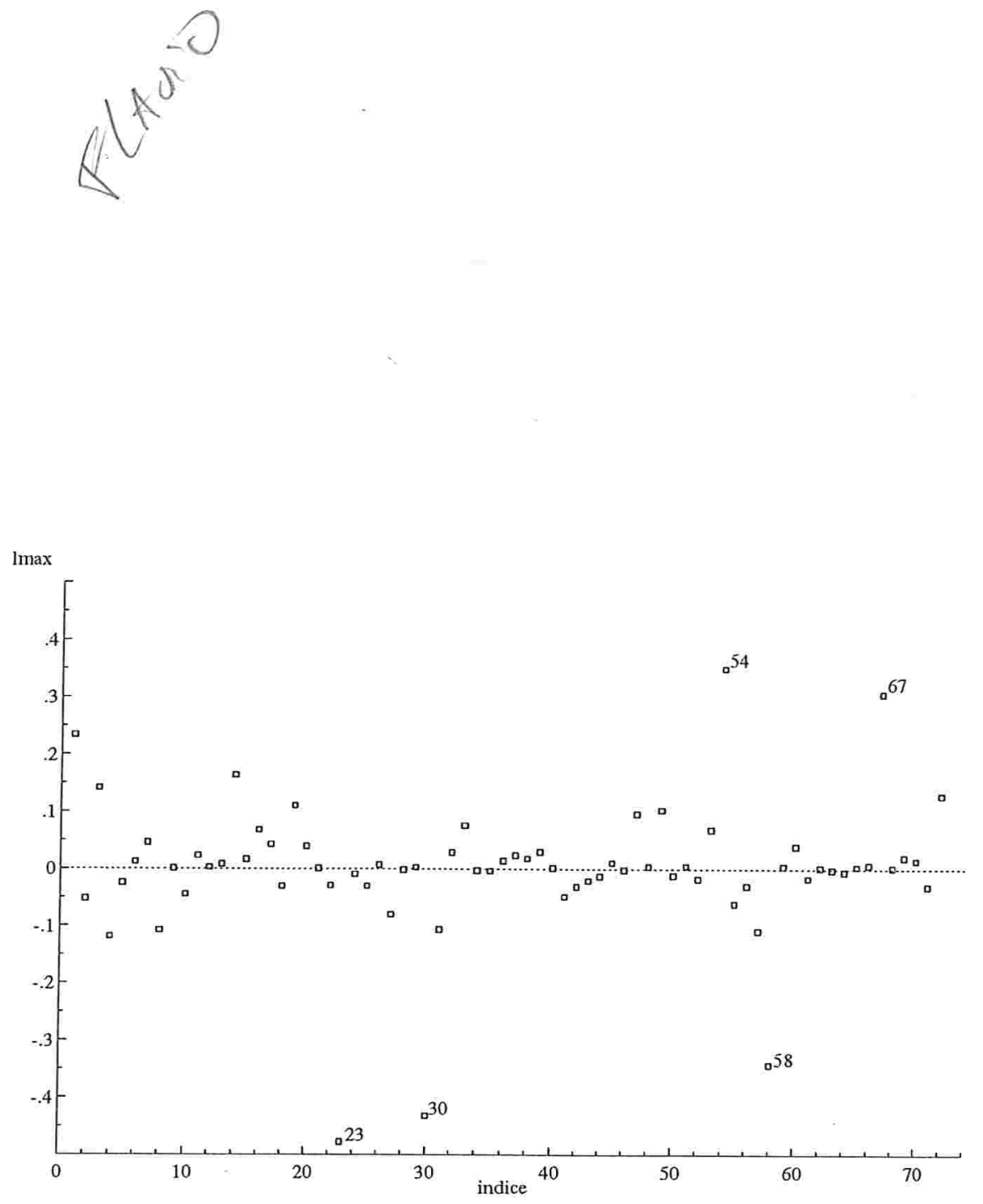

Figura 3.10: Gráfico de índices de $\boldsymbol{l}_{\max }$ na perturbação da ponderação de casos, modelo heteroscedástico - Exemplo 3.2. 


\section{Capítulo 4}

\section{Modelos Estruturais para Várias Populações}

\subsection{Introdução}

Iniciamos com o modelo de regressão linear simples estrutural normal com erro de medição na covariável. A relação entre a resposta $y$ e a covariável $x$, tendo $n$ valores em $k$ grupos $(k \geq 2)$ é dada por

$$
y_{i j}=\alpha+\beta x_{i j}, j=1, \ldots, n, i=1, \ldots, k,
$$

com $x_{i j} \sim \mathrm{N}\left(\mu_{x}, \sigma_{x x}\right)$, sendo que " $\sim \mathrm{N}\left(\mu_{x}, \sigma_{x x}\right)$ " representa variáveis aleatórias normalmente distribuídas com média e variância iguais a $\mu_{x}$ e $\sigma_{x x}$, respectivamente. As variáveis $y$ e $x$ não são observáveis exatamente, mas acrescidas de erros de medição através de

$$
Y_{i j}=y_{i j}+e_{i j} \text { e } X_{i j}=x_{i j}+u_{i j},
$$

com $e_{i j} \sim \mathrm{N}\left(0, \sigma_{e e}\right), u_{i j} \sim \mathrm{N}\left(0, \sigma_{u u}\right)$ e

$$
\left\{\left(x_{i j}, e_{i j}, u_{i j}\right), j=1, \ldots, n, \quad i=1, \ldots, k\right\} \text { independentes. }
$$

Os seis parâmetros são representados pelo vetor

$$
\boldsymbol{\theta}=\left(\mu_{x}, \alpha, \beta, \sigma_{u u}, \sigma_{e e}, \sigma_{x x}\right)^{\prime},
$$

onde "'" denota transposição. Este modelo é inidentificável. Fuller (1987) e Cheng \& Van Ness (1999) discutem o problema de identificação e vários outros tópicos 
pertinentes a este modelo. Apesar de termos um só grupo $(k=1)$, adotamos a notação geral também para este modelo. Em seguida apresentamos generalizações, sendo que em todas elas a suposição (4.1) é mantida.

Cox (1976) e Dolby (1976) estudam um modelo em que as médias da covariável são diferentes nos diversos grupos, ou seja, $x_{i j} \sim \mathrm{N}\left(\mu_{x i}, \sigma_{x x}\right), \boldsymbol{\theta}=\left(\boldsymbol{\mu}_{x}^{\prime}, \alpha, \beta, \sigma_{u u}, \sigma_{e e}, \sigma_{x x}\right)^{\prime}$, com $\boldsymbol{\mu}_{x}=\left(\mu_{x 1}, \ldots, \mu_{x k}\right)^{\prime}$. O método de máxima verossimilhança fornece estimadores consistentes para os parâmetros sem necessidade de informações adicionais comumente incorporadas - por exemplo, razão das variâncias dos erros de medição $\left(\sigma_{e e} / \sigma_{u u}\right)$ conhecida (Fuller, 1987; Cheng \& Van Ness, 1999) - , mas nem sempre disponíveis. Os autores apresentam ainda expressões para as variâncias assintóticas dos estimadores. Um teste sobre o coeficiente da covariável $(\beta)$ é proposto por Dolby (1976).

Brown (1978) estende a formulação anterior admitindo heterogeneidade não apenas das médias da covariável, mas também das variâncias desta e das variâncias dos erros de medição, isto é, $x_{i j} \sim \mathrm{N}\left(\mu_{x i}, \sigma_{x x i}\right), u_{i j} \sim \mathrm{N}\left(0, \sigma_{u u i}\right)$ e $e_{i j} \sim \mathrm{N}\left(0, \sigma_{e e i}\right)$, com vetor paramétrico $\theta=\left(\boldsymbol{\mu}_{x}^{\prime}, \alpha, \beta, \boldsymbol{\sigma}_{u u}^{\prime}, \boldsymbol{\sigma}_{e e}^{\prime}, \boldsymbol{\sigma}_{x x}^{\prime}\right)^{\prime}$. São apresentados estimadores de mínimos quadrados generalizados e comparações com máxima verossimilhança.

A relação estudada por Wong (1991) permite que os valores de $\mu_{x}, \alpha, \beta, \sigma_{u u}, \sigma_{e e}$ e $\sigma_{x x}$ difiram entre os grupos, generalizando ao máximo o modelo linear simples. Resumidamente escrevemos

$$
\begin{gathered}
y_{i j}=\alpha_{i}+\beta_{i} x_{i j}, \quad x_{i j} \sim \mathrm{N}\left(\mu_{x i}, \sigma_{x x i}\right), \\
Y_{i j}=y_{i j}+e_{i j}, \quad X_{i j}=x_{i j}+u_{i j}, \\
e_{i j} \sim \mathrm{N}\left(0, \sigma_{e e i}\right) \quad \text { e } u_{i j} \sim \mathrm{N}\left(0, \sigma_{u u i}\right),
\end{gathered}
$$

$j=1, \ldots, n, i=1, \ldots, k$. Temos $6 k$ parâmetros no vetor $\boldsymbol{\theta}=\left(\boldsymbol{\mu}_{x}^{\prime}, \boldsymbol{\alpha}^{\prime}, \boldsymbol{\beta}^{\prime}, \boldsymbol{\sigma}_{u u}^{\prime}, \boldsymbol{\sigma}_{e e}^{\prime}, \boldsymbol{\sigma}_{x x}^{\prime}\right)^{\prime}$, que é inidentificável. O problema é resolvido supondo $\sigma_{e e i} / \sigma_{u u i}$ conhecidas, $i=$ $1, \ldots, k$. Como existem seis diferentes tipos de parâmetros, se percorrermos todas as situações desde a homogeneidade total nos parâmetros (Fuller, 1987; Cheng \& Van Ness, 1999) até a heterogeneidade total (Wong, 1991), enumeramos 64 modelos listados na Tabela 4.1, convencionando que "o" e "๑" indicam que um dado tipo de parâmetro assume o mesmo valor e valores diferentes, respectivamente, nos $k$ grupos. Exemplificando, os modelos vistos aqui recebem os rótulos 1, 33, 40 e 64 .

Nosso interesse estará voltado para modelos nos quais seja possível obter estimadores consistentes de posse apenas das observações $\left\{\left(Y_{i j}, X_{i j}\right), j=1, \ldots, n, i=\right.$ 
Tabela 4.1: Enumeração dos modelos estruturais entre vários grupos.

\begin{tabular}{|c|c|c|c|c|c|c|c|c|c|c|c|c|c|}
\hline \multirow[b]{2}{*}{ Modelo } & \multicolumn{6}{|c|}{ Parâmetros } & \multirow[b]{2}{*}{ Modelo } & \multicolumn{6}{|c|}{ Parâmetros } \\
\hline & $\mu_{x}$ & $\alpha$ & $\beta$ & $\sigma_{u u}$ & $\sigma_{e e}$ & $\sigma_{x x}$ & & $\mu_{x}$ & $\alpha$ & $\beta$ & $\sigma_{u u}$ & $\sigma_{e e}$ & $\sigma_{x x}$ \\
\hline 1 & 0 & 0 & 0 & 0 & 0 & 0 & 33 & - & 0 & 0 & 0 & $\mathrm{O}$ & 0 \\
\hline 2 & o & O & 0 & 0 & o & - & 34 & - & 0 & o & o & 0 & - \\
\hline 3 & 0 & 0 & 0 & 0 & • & o & 35 & - & 0 & o & o & - & o \\
\hline 4 & o & o & o & $\mathrm{O}$ & - & - & 36 & - & o & o & o & - & $\bullet$ \\
\hline 5 & $\mathrm{O}$ & 0 & $\mathrm{o}$ & - & o & 0 & 37 & - & o & 0 & - & o & 0 \\
\hline 6 & $\mathrm{O}$ & o & o & - & o & - & 38 & - & o & 0 & - & o & - \\
\hline 7 & o & $\mathrm{O}$ & 0 & - & - & 0 & 39 & - & 0 & 0 & - & • & 0 \\
\hline 8 & o & o & 0 & • & $\bullet$ & • & 40 & • & 0 & 0 & - & • & 0 \\
\hline 9 & 0 & o & - & 0 & 0 & 0 & 41 & • & 0 & • & 0 & 0 & 0 \\
\hline 10 & 0 & o & - & 0 & 0 & - & 42 & - & 0 & • & 0 & 0 & • \\
\hline 11 & 0 & 0 & • & 0 & - & 0 & 43 & - & 0 & • & 0 & • & 0 \\
\hline 12 & 0 & 0 & - & 0 & - & • & 44 & - & 0 & • & 0 & - & - \\
\hline 13 & 0 & 0 & • & - & o & 0 & 45 & - & 0 & • & • & 0 & 0 \\
\hline 14 & o & 0 & • & - & 0 & - & 46 & • & 0 & - & - & 0 & - \\
\hline 15 & 0 & 0 & - & - & - & 0 & 47 & - & 0 & $\bullet$ & • & - & 0 \\
\hline 16 & O & $\mathrm{O}$ & - & - & - & - & 48 & • & 0 & • & • & - & • \\
\hline 17 & 0 & - & 0 & 0 & 0 & 0 & 49 & - & • & 0 & 0 & 0 & 0 \\
\hline 18 & 0 & • & 0 & 0 & 0 & - & 50 & - & $\bullet$ & 0 & 0 & 0 & • \\
\hline 19 & 0 & - & 0 & 0 & - & o & 51 & - & • & o & o & - & 0 \\
\hline 20 & 0 & - & 0 & 0 & - & - & 52 & - & - & o & o & • & • \\
\hline 21 & 0 & $\bullet$ & 0 & - & 0 & 0 & 53 & - & - & 0 & • & 0 & O \\
\hline 22 & 0 & - & 0 & - & 0 & - & 54 & - & • & 0 & • & 0 & - \\
\hline 23 & 0 & - & 0 & - & • & 0 & 55 & - & • & 0 & • & $\bullet$ & 0 \\
\hline 24 & 0 & - & 0 & - & - & • & 56 & - & $\bullet$ & 0 & • & • & • \\
\hline 25 & 0 & - & - & 0 & 0 & 0 & 57 & - & - & • & 0 & 0 & 0 \\
\hline 26 & 0 & - & - & 0 & 0 & • & 58 & - & • & • & 0 & 0 & - \\
\hline 27 & 0 & - & - & 0 & - & 0 & 59 & - & • & • & o & - & 0 \\
\hline 28 & 0 & • & - & 0 & - & - & 60 & • & - & • & o & - & - \\
\hline 29 & 0 & - & - & • & O & 0 & 61 & • & - & - & • & 0 & 0 \\
\hline 30 & 0 & $\cdot$ & - & - & o & - & 62 & - & - & 0 & • & 0 & - \\
\hline 31 & 0 & - & - & • & - & 0 & 63 & • & - & - & • & • & 0 \\
\hline 32 & 0 & - & - & - & - & - & 64 & - & - & - & - & - & - \\
\hline
\end{tabular}


$1, \ldots, k\}$. Nas seções seguintes apresentamos resultados sobre a estimação por máxima verossimilhança (MV), um teste de ajuste dos modelos, as variâncias assintóticas dos estimadores de MV e um estudo de influência local. Um exemplo com dados reais encontra-se na Seção 4.5. Alguns pontos para trabalhos futuros são listados na Seção 4.6 .

\subsection{Estimação}

Devemos primeiramente nos preocupar com a identificabilidade. Conforme Cheng \& Van Ness $(1999$, p. 7), no modelo estrutural a identificabilidade de um parâmetro equivale à existência de um estimador consistente para o parâmetro. No caso da distribuição normal, Fuller (1987, p. 352) ressalta que uma condição necessária para que um modelo seja identificável é que o número de elementos na distribuição conjunta das observações iguale ou exceda o número de parâmetros no modelo, lembrando que os elementos do vetor de médias e da matriz de covariâncias determinam unicamente uma distribuição normal multivariada. Tomamos o modelo 18 para fins de ilustração. Temos $2 k+4$ parâmetros no vetor $\theta=\left(\mu_{x}, \alpha^{\prime}, \beta, \sigma_{u u}, \sigma_{e e}, \boldsymbol{\sigma}_{x x}^{\prime}\right)^{\prime}$ e as observações são tais que

$$
\left(\begin{array}{c}
Y_{i j} \\
X_{i j}
\end{array}\right) \sim \mathrm{N}_{2}\left(\left(\begin{array}{c}
\mu_{Y i} \\
\mu_{x}
\end{array}\right),\left(\begin{array}{ll}
\sigma_{Y Y i} & \sigma_{Y X i} \\
& \sigma_{X X i}
\end{array}\right)\right),
$$

$\operatorname{com} \mu_{Y i}=\alpha_{i}+\beta \mu_{x}, \sigma_{Y Y i}=\beta^{2} \sigma_{x x i}+\sigma_{e e}, \sigma_{Y X i}=\beta \sigma_{x x i}$ e $\sigma_{X X i}=\sigma_{x x i}+\sigma_{u u}, j=$ $1, \ldots, n, i=1, \ldots, k$. Conseqüentemente, a distribuição conjunta de $\left\{\left(Y_{i j}, X_{i j}\right), j=\right.$ $1, \ldots, n, i=1, \ldots, k\}$ envolve $4 k+1$ elementos distintos dados por

$$
\mu_{x} \text { e }\left\{\left(\mu_{Y i}, \sigma_{Y Y i}, \sigma_{Y X i}, \sigma_{X X i}\right), i=1, \ldots, k\right\}
$$

de modo que a condição necessária está satisfeita. Tratamos analogamente o restante da Tabela 4.2, na qual "Número de elementos" é o número de elementos que definem a distribuição conjunta das observações e "(*)" sinaliza modelos inidentificáveis, pois violam a condição acima.

Na Tabela 4.2 contamos 37 modelos satisfazendo a condição necessária à identificabilidade enunciada. De fato, em todos eles existem estimadores consistentes do tipo método dos momentos para o vetor $\theta$. Sejam $m_{Y Y i}, m_{Y X i}$ e $m_{X X i}$ as covariâncias 
Tabela 4.2: Identificabilidade dos modelos estruturais entre vários grupos.

\begin{tabular}{|c|c|c|c|c|c|}
\hline Modelo & $\begin{array}{l}\text { Número de } \\
\text { parâmetros }\end{array}$ & $\begin{array}{l}\text { Número de } \\
\text { elementos }\end{array}$ & Modelo & $\begin{array}{l}\text { Número de } \\
\text { parâmetros }\end{array}$ & $\begin{array}{l}\text { Número de } \\
\text { elementos }\end{array}$ \\
\hline $1(*)$ & 6 & 5 & 33 & $k+5$ & $2 k+3$ \\
\hline 2 & $k+5$ & $3 k+2$ & 34 & $2 k+4$ & $5 k$ \\
\hline $3(*)$ & $k+5$ & $k+4$ & 35 & $2 k+4$ & $3 k+2$ \\
\hline 4 & $2 k+4$ & $3 k+2$ & 36 & $3 k+3$ & $5 k$ \\
\hline $5(*)$ & $k+5$ & $k+4$ & 37 & $2 k+4$ & $3 k+2$ \\
\hline 6 & $2 k+4$ & $3 k+2$ & 38 & $3 k+3$ & $5 k$ \\
\hline $7(*)$ & $2 k+4$ & $2 k+3$ & 39 & $3 k+3$ & $4 k+1$ \\
\hline $8(*)$ & $3 k+3$ & $3 k+2$ & 40 & $4 k+2$ & $5 k$ \\
\hline 9 & $k+5$ & $3 k+2$ & 41 & $2 k+4$ & $4 k+1$ \\
\hline 10 & $2 k+4$ & $4 k+1$ & 42 & $3 k+3$ & $5 k$ \\
\hline 11 & $2 k+4$ & $3 k+2$ & 43 & $3 k+3$ & $4 k+1$ \\
\hline 12 & $3 k+3$ & $4 k+1$ & 44 & $4 k+2$ & $5 k$ \\
\hline 13 & $2 k+4$ & $4 k+1$ & 45 & $3 k+3$ & $5 k$ \\
\hline 14 & $3 k+3$ & $4 k+1$ & 46 & $4 k+2$ & $5 k$ \\
\hline 15 & $3 k+3$ & $4 k+1$ & 47 & $4 k+2$ & $5 k$ \\
\hline $16\left(^{*}\right)$ & $4 k+2$ & $4 k+1$ & $48\left(^{*}\right)$ & $5 k+1$ & $5 k$ \\
\hline $17(*)$ & $k+5$ & $k+4$ & $49\left(^{*}\right)$ & $2 k+4$ & $2 k+3$ \\
\hline 18 & $2 k+4$ & $4 k+1$ & 50 & $3 k+3$ & $5 k$ \\
\hline $19\left(^{*}\right)$ & $2 k+4$ & $2 k+3$ & $51\left(^{*}\right)$ & $3 k+3$ & $3 k+2$ \\
\hline 20 & $3 k+3$ & $4 k+1$ & 52 & $4 k+2$ & $5 k$ \\
\hline $21\left(^{*}\right)$ & $2 k+4$ & $2 k+3$ & $53(*)$ & $3 k+3$ & $3 k+2$ \\
\hline 22 & $3 k+3$ & $4 k+1$ & 54 & $4 k+2$ & $5 k$ \\
\hline $23\left(^{*}\right)$ & $3 k+3$ & $3 k+2$ & $55\left(^{*}\right)$ & $4 k+2$ & $4 k+1$ \\
\hline $24(*)$ & $4 k+2$ & $4 k+1$ & $56\left({ }^{*}\right)$ & $5 k+1$ & $5 k$ \\
\hline 25 & $2 k+4$ & $3 k+2$ & 57 & $3 k+3$ & $4 k+1$ \\
\hline 26 & $3 k+3$ & $4 k+1$ & 58 & $4 k+2$ & $5 k$ \\
\hline $27(*)$ & $3 k+3$ & $3 k+2$ & $59\left({ }^{*}\right)$ & $4 k+2$ & $4 k+1$ \\
\hline $28\left(^{*}\right)$ & $4 k+2$ & $4 k+1$ & $60\left(^{*}\right)$ & $5 k+1$ & $5 k$ \\
\hline 29 & $3 k+3$ & $4 k+1$ & 61 & $4 k+2$ & $5 k$ \\
\hline $30\left(^{*}\right)$ & $4 k+2$ & $4 k+1$ & $62\left({ }^{*}\right)$ & $5 k+1$ & $5 k$ \\
\hline $31\left(^{*}\right)$ & $4 k+2$ & $4 k+1$ & $63\left(^{*}\right)$ & $5 k+1$ & $5 k$ \\
\hline $32\left(^{*}\right)$ & $5 k+1$ & $4 k+1$ & $64\left(^{*}\right)$ & $6 k$ & $5 k$ \\
\hline
\end{tabular}

Nota: $\left(^{*}\right)$ sinaliza modelo inidentificável. 
amostrais do $i$-ésimo grupo, $i=1, \ldots, k$, onde, por exemplo,

$$
m_{Y X i}=\frac{1}{n-1} \sum_{j=1}^{n}\left(Y_{i j}-\bar{Y}_{i .}\right)\left(X_{i j}-\bar{X}_{i .}\right)
$$

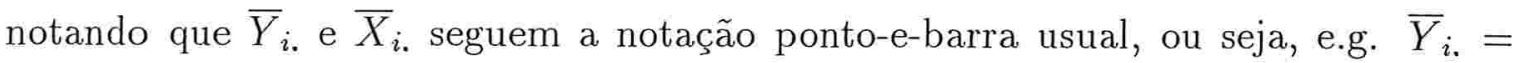
$\sum_{j=1}^{n} Y_{i j} / n$. No modelo 18 um conjunto de estimadores consistentes é dado por

$$
\begin{gathered}
\widehat{\mu_{x}}=\frac{1}{k n} \sum_{i=1}^{k} \sum_{j=1}^{n} X_{i j}=\bar{X}_{. .} \quad, \quad \widehat{\beta}=\frac{2}{k(k-1)} \sum_{i=1}^{k} \sum_{l=i+1}^{k} \frac{m_{Y X i}-m_{Y X l}}{m_{X X i}-m_{X X l}}, \\
{\widehat{\sigma_{x x i}}}^{*}=\frac{m_{Y X i}}{\widehat{\beta}}, \quad \bar{Y}_{i .}-\widehat{\beta} \widehat{\mu_{x x}}={\widehat{\sigma_{x x i}}}^{*} I_{(0, \infty)}\left({\widehat{\sigma_{x x i}}}^{*}\right)+\epsilon I_{(-\infty, 0]}\left({\widehat{\sigma_{x x i}}}^{*}\right), \quad i=1, \ldots, k, \\
{\widehat{\sigma_{u u}}}^{*}=\frac{1}{k} \sum_{i=1}^{k}\left(m_{X X i}-\frac{\left.m_{Y X i}\right)}{\widehat{\beta}}\right), \quad \widehat{\sigma_{u u}}={\widehat{\sigma_{u u}}}^{*} I_{(0, \infty)}\left({\widehat{\sigma_{u u}}}^{*}\right)+\epsilon I_{(-\infty, 0]}\left({\widehat{\sigma_{u u}}}^{*}\right)
\end{gathered}
$$

$\mathrm{e}$

$$
{\widehat{\sigma_{e e}}}^{*}=\frac{1}{k} \sum_{i=1}^{k}\left(m_{Y Y i}-\widehat{\beta} m_{Y X i}\right) \quad, \quad \widehat{\sigma_{e e}}={\widehat{\sigma_{e e}}}^{*} I_{(0, \infty)}\left({\widehat{\sigma_{e e}}}^{*}\right)+\epsilon I_{(-\infty, 0]}\left({\widehat{\sigma_{e e}}}^{*}\right),
$$

onde $I($.$) é a função indicador e \epsilon>0$ é uma constante.

Estatísticas suficientes desempenham um papel central na estimação paramétrica. No nosso caso, examinando o modelo 1 concluiremos sobre todos os outros. Temos

$$
\left(\begin{array}{c}
Y_{i j} \\
X_{i j}
\end{array}\right) \stackrel{i i d}{\sim} \mathrm{N}_{2}\left(\left(\begin{array}{c}
\alpha+\beta \mu_{x} \\
\mu_{x}
\end{array}\right), \Sigma\right)
$$

onde

$$
\Sigma=\left(\begin{array}{cc}
\beta^{2} \sigma_{x x}+\sigma_{e e} & \beta \sigma_{x x} \\
& \sigma_{x x}+\sigma_{u u}
\end{array}\right)
$$

de forma que

$$
\begin{aligned}
f\left(Y_{i j}, X_{i j} ; \boldsymbol{\theta}\right)= & \kappa(\boldsymbol{\theta}) \exp \left\{-\frac{\sigma_{x x}+\sigma_{u u}}{2|\Sigma|} Y_{i j}^{2}+\frac{\sigma_{u u}\left(\alpha+\beta \mu_{x}\right)+\alpha \sigma_{x x}}{|\Sigma|} Y_{i j}\right. \\
& \left.+\frac{\beta \sigma_{x x}}{|\Sigma|} X_{i j} Y_{i j}+\frac{\mu_{x} \sigma_{e e}-\alpha \beta \sigma_{x x}}{|\Sigma|} X_{i j}-\frac{\beta^{2} \sigma_{x x}+\sigma_{e e}}{|\Sigma|} X_{i j}^{2}\right\}
\end{aligned}
$$

$j=1, \ldots, n, i=1, \ldots, k$, onde $\kappa$ é uma função apenas de $\theta$ e $|\Sigma|=\left(\beta^{2} \sigma_{u u}+\right.$ $\left.\sigma_{e e}\right) \sigma_{x x}+\sigma_{u u} \sigma_{e e}$. Podemos agora apontar estatísticas suficientes. No modelo 33 o 
número de estatísticas suficientes minimais é $2 k+3\left(\sum_{i, j} Y_{i j}^{2}, \sum_{i, j} X_{i, j} Y_{i, j}, \sum_{i, j} X_{i, j}^{2}\right.$, $\left.\left\{\sum_{j} Y_{i j}, \sum_{j} X_{i, j}\right\}, i=1, \ldots, k\right)$, enquanto nos demais este número é $5 k\left(\left\{\sum_{j} Y_{i j}^{2}\right.\right.$, $\left.\left.\sum_{j} X_{i, j} Y_{i, j}, \sum_{j} X_{i, j}^{2}, \sum_{j} Y_{i j}, \sum_{j} X_{i, j}\right\}, i=1, \ldots, k\right)$. Portanto, para $k>2$ todos os 37 modelos pertencem à família exponencial curvada (Lehmann, 1997, p. 45). Em termos práticos, isto nos diz que determinar os estimadores de máxima verossimilhança (MV) não se reduz a resolver o sistema de equações formado ao igualarmos as esperanças das estatísticas suficientes às respectivas estatísticas.

Em seguida desenvolvemos expressões para a função log-verossimilhança e suas derivadas de primeira e segunda ordens (Seção 4.2.1) em relação a $\boldsymbol{\theta}$. O modelo 64 serve como base, proporcionando uma formulação geral diretamente particularizável a cada um dos 37 modelos da Tabela 4.2. Temos

$$
\left(\begin{array}{c}
Y_{i j} \\
X_{i j}
\end{array}\right) \stackrel{\text { indep. }}{\sim} \mathrm{N}_{2}\left(\left(\begin{array}{c}
\mu_{Y i} \\
\mu_{X i}
\end{array}\right), \Sigma_{i}\right)
$$

com

$$
\mu_{Y i}=\mathrm{E}\left(Y_{i j}\right)=\alpha_{i}+\beta_{i} \mu_{x i}, \mu_{X i}=\mathrm{E}\left(X_{i j}\right)=\mu_{x i}
$$

e

$$
\Sigma_{i}=\left(\begin{array}{cc}
\beta_{i}^{2} \sigma_{x x i}+\sigma_{e e i} & \beta_{i} \sigma_{x x i} \\
& \sigma_{x x i}+\sigma_{u u i}
\end{array}\right),
$$

$j=1, \ldots, n, i=1, \ldots, k$. Fazendo

$$
\boldsymbol{Y}_{j}=\left(Y_{1 j}, \ldots, Y_{k j}\right)^{\prime} \text { e } \boldsymbol{X}_{j}=\left(X_{1 j}, \ldots, X_{k j}\right)^{\prime}
$$

passamos a ter

$$
\left(\begin{array}{l}
\boldsymbol{Y}_{j} \\
\boldsymbol{X}_{j}
\end{array}\right) \stackrel{\text { iid }}{\sim} \mathrm{N}_{2 k}\left(\left(\begin{array}{l}
\boldsymbol{\mu}_{y} \\
\boldsymbol{\mu}_{x}
\end{array}\right), \mathbf{V}\right), j=1, \ldots, n
$$

onde

$$
\boldsymbol{\mu}_{y}=\alpha+\beta \odot \boldsymbol{\mu}_{x} \quad, \quad \mathrm{~V}=\left(\begin{array}{cc}
\mathrm{D}\left(\boldsymbol{\sigma}_{e e}+\beta \odot \beta \odot \boldsymbol{\sigma}_{x x}\right) & \mathrm{D}\left(\boldsymbol{\beta} \odot \boldsymbol{\sigma}_{x x}\right) \\
& \mathrm{D}\left(\boldsymbol{\sigma}_{u u}+\boldsymbol{\sigma}_{x x}\right)
\end{array}\right),
$$

" $\odot$ " denota o produto de Hadamard (Styan, 1973) e $\mathbf{D}\left(\boldsymbol{\beta} \odot \boldsymbol{\sigma}_{x x}\right)$ é a matriz diagonal com elemento $\beta_{i} \sigma_{x x i}$ na diagonal principal, $i=1, \ldots, k$. Sejam $\boldsymbol{Y}=\left(\boldsymbol{Y}_{1}^{\prime}, \ldots, \boldsymbol{Y}_{n}^{\prime}\right)^{\prime}$, $\boldsymbol{X}=\left(\boldsymbol{X}_{1}^{\prime}, \ldots, \boldsymbol{X}_{n}^{\prime}\right)^{\prime}$ e $\boldsymbol{d}_{j}=\left(\begin{array}{c}\boldsymbol{d}_{j Y} \\ \boldsymbol{d}_{j X}\end{array}\right)=\left(\begin{array}{c}\boldsymbol{Y}_{j-\boldsymbol{\mu}_{y}} \\ \boldsymbol{X}_{j-} \boldsymbol{\mu}_{x}\end{array}\right), j=1, \ldots, n$. A função $\log$ verossimilhança é dada por

$$
\log \mathcal{L}(\boldsymbol{\theta} ; \boldsymbol{Y}, \boldsymbol{X})=-n k \log (2 \pi)-\frac{1}{2} n \log |\mathrm{V}|-\frac{1}{2} \sum_{j=1}^{n} \boldsymbol{d}_{j}^{\prime} \mathrm{V}^{-1} \boldsymbol{d}_{j},
$$


onde $|\mathbf{V}|=\prod_{i=1}^{k} \delta_{i}, \quad \delta_{i}=\left|\Sigma_{i}\right|=\left(\beta_{i}^{2} \sigma_{u u i}+\sigma_{e e i}\right) \sigma_{x x i}+\sigma_{u u i} \sigma_{e e i} \mathrm{e}$

$$
\mathrm{V}^{-1}=\left(\begin{array}{cc}
\mathrm{D}\left(\left(\sigma_{u u}+\sigma_{x x}\right) \oslash \delta\right) & -\mathrm{D}\left(\beta \odot \sigma_{x x} \oslash \delta\right) \\
& \mathrm{D}\left(\left(\sigma_{e e}+\beta \odot \beta \odot \sigma_{x x}\right) \oslash \delta\right)
\end{array}\right)
$$

sendo que " $\oslash$ " representa a divisão elemento a elemento e $\delta=\left(\delta_{1}, \ldots, \delta_{k}\right)^{\prime}$. O espaço paramétrico é determinado por $\mu_{x i} \in \mathbb{R}, \alpha_{i} \in \mathbb{R}, \beta_{i} \in \mathbb{R}, \sigma_{u u i} \geq 0, \sigma_{e e i} \geq 0, \sigma_{x x i} \geq 0$ e $\delta_{i}>0, i=1, \ldots, k$.

Seja $\mathbf{1}_{k}$ o vetor unitário $k$-dimensional. No modelo 18 , fazendo

$$
\boldsymbol{\mu}_{x}=\mu_{x} \mathbf{1}_{k}, \boldsymbol{\beta}=\beta \mathbf{1}_{k}, \boldsymbol{\sigma}_{u u}=\sigma_{u u} \mathbf{1}_{k} \text { e } \boldsymbol{\sigma}_{e e}=\sigma_{e e} \mathbf{1}_{k}
$$

podemos recorrer a (4.5) para o cálculo de $\log \mathcal{L}$.

Sejam $v$ e $\theta_{r}$ elementos genéricos de $\mathbf{V}$ e $\theta$, respectivamente, $\mathbf{V}_{\theta_{r}}$ a matriz $2 k \times 2 k$ com elemento $\frac{\partial}{\partial \theta_{r}} v, \boldsymbol{d}_{\theta_{r}}$ o vetor $2 k \times 1$ com expressão

$$
\boldsymbol{d}_{\theta_{r}}=\frac{\partial}{\partial \theta_{r}} \overline{\boldsymbol{d}}_{.}=\frac{\partial}{\partial \theta_{r}}\left(\begin{array}{c}
\overline{\boldsymbol{d}}_{. Y} \\
\overline{\boldsymbol{d}}_{. X}
\end{array}\right)=\frac{\partial}{\partial \theta_{r}}\left(\begin{array}{c}
\overline{\boldsymbol{Y}} \cdot-\boldsymbol{\alpha}-\boldsymbol{\beta} \odot \boldsymbol{\mu}_{x} \\
\overline{\boldsymbol{X}}_{.}-\boldsymbol{\mu}_{x}
\end{array}\right),
$$

$\mathbf{M}$ a matriz $2 k \times 2 k$ obtida de $\sum_{j=1}^{n} \boldsymbol{d}_{j} \boldsymbol{d}_{j}^{\prime} / n$ e $\mathbf{P}=\mathbf{V}^{-1}(\mathbf{M}-\mathbf{V}) \mathbf{V}^{-1}$. Dolby (1976) mostrou que

$$
\frac{\partial}{\partial \theta_{r}} \log \mathcal{L}(\boldsymbol{\theta} ; \boldsymbol{Y}, \boldsymbol{X})=n\left[\frac{1}{2} \operatorname{tr}\left(\mathbf{P} \mathbf{V}_{\theta_{r}}\right)-\boldsymbol{d}_{\theta_{r}}^{\prime} \mathbf{V}^{-1} \overline{\boldsymbol{d}} .\right]
$$

onde "tr" indica o traço de uma matriz. Notamos em seguida que (4.6) se adapta ao cálculo do vetor gradiente em qualquer um dos modelos da Tabela 4.2. Rearranjamos o vetor $\theta$ como $\varphi=\left(\varphi_{1}^{\prime}, \ldots, \varphi_{k}^{\prime}\right)^{\prime}$, onde $\varphi_{i}=\left(\mu_{x i}, \alpha_{i}, \beta_{i}, \sigma_{u u i}, \sigma_{e e i}, \sigma_{x x i}\right)^{\prime}, i=1, \ldots, k$. Definindo $g_{1}\left(\varphi_{i}\right)=-n \log \left(\delta_{i}\right) / 2 \mathrm{e}$

$$
\begin{aligned}
g_{2}\left(\varphi_{i}, Y_{i j}, X_{i j}\right)= & -\frac{1}{2 \delta_{i}}\left[\left(\sigma_{u u i}+\sigma_{x x i}\right)\left(Y_{i j}-\alpha_{i}-\beta_{i} \mu_{x i}\right)^{2}\right. \\
& -2 \beta_{i} \sigma_{x x i}\left(Y_{i j}-\alpha_{i}-\beta_{i} \mu_{x i}\right)\left(X_{i j}-\mu_{x i}\right) \\
& \left.+\left(\sigma_{e e i}+\beta_{i}^{2} \sigma_{x x i}\right)\left(X_{i j}-\mu_{x i}\right)^{2}\right],
\end{aligned}
$$

(4.5) passa a ser

$$
\log \mathcal{L}(\boldsymbol{\varphi} ; \boldsymbol{Y}, \boldsymbol{X})=-n k \log (2 \pi)+\sum_{i=1}^{k} g_{1}\left(\boldsymbol{\varphi}_{i}\right)+\sum_{i=1}^{k} \sum_{j=1}^{n} g_{2}\left(\boldsymbol{\varphi}_{i}, Y_{i j}, X_{i j}\right)
$$


Como exemplo,

$$
\frac{\partial}{\partial \boldsymbol{\beta}} \log \mathcal{L}=\left(\begin{array}{c}
\frac{\partial}{\partial \beta_{1}} g_{1}\left(\varphi_{1}\right)+\sum_{j=1}^{n} \frac{\partial}{\partial \beta_{1}} g_{2}\left(\varphi_{1}, Y_{1 j}, X_{1 j}\right) \\
\vdots \\
\frac{\partial}{\partial \beta_{k}} g_{1}\left(\varphi_{k}\right)+\sum_{j=1}^{n} \frac{\partial}{\partial \beta_{k}} g_{2}\left(\varphi_{k}, Y_{k j}, X_{k j}\right)
\end{array}\right)=\left(\begin{array}{c}
a_{1} \\
\vdots \\
a_{k}
\end{array}\right) .
$$

Se tivermos $\beta_{1}=\cdots=\beta_{k}=\beta$, então

$$
\begin{aligned}
\frac{\partial}{\partial \beta} \log \mathcal{L} & =\sum_{i=1}^{k} \frac{\partial}{\partial \beta} g_{1}\left(\varphi_{i}\right)+\sum_{i=1}^{k} \sum_{j=1}^{n} \frac{\partial}{\partial \beta} g_{2}\left(\varphi_{i}, Y_{i j}, X_{i j}\right) \\
& =\left.\left(a_{1}+\cdots+a_{k}\right)\right|_{\beta_{1}=\cdots=\beta_{k}=\beta}
\end{aligned}
$$

ou seja, podemos usar (4.6) e somar os elementos do vetor $\frac{\partial}{\partial \beta} \log \mathcal{L}$. A aplicação de (4.6) aos elementos de $\theta$ no modelo 64 nos leva a um vetor $6 k \times 1$. Em um modelo qualquer, $\frac{\partial}{\theta} \log \mathcal{L}$ deve ser pré-multiplicado por

$$
\Lambda=\operatorname{bdiag}\left(\lambda_{\mu_{x}}, \lambda_{\alpha}, \lambda_{\beta}, \lambda_{\sigma_{u u}}, \lambda_{\sigma_{e e}}, \lambda_{\sigma_{x x}}\right),
$$

onde "bdiag" denota uma matriz diagonal em blocos e $\boldsymbol{\lambda}_{\mu_{x}}=\mathbf{I}_{k}$ se houver heterogeneidade de $\mu_{x}$ nos $k$ grupos; $\boldsymbol{\lambda}_{\mu_{x}}=\mathbf{1}_{k}^{\prime}$, caso contrário. A dimensão de $\boldsymbol{\Lambda}$ é $p \times 6 k$, onde $p$ é o número de parâmetros do modelo. No modelo $18, p=2 k+4 \mathrm{e}$ $\Lambda=\operatorname{bdiag}\left(\mathbf{1}_{k}^{\prime}, \mathbf{I}_{k}, \mathbf{1}_{k}^{\prime}, \mathbf{1}_{k}^{\prime}, \mathbf{1}_{k}^{\prime}, \mathbf{I}_{k}\right)$.

Em (4.6) a primeira parcela se anula para elementos de $\left(\boldsymbol{\mu}_{x}^{\prime}, \boldsymbol{\alpha}^{\prime}\right)^{\prime}$, enquanto a segunda para elementos de $\left(\boldsymbol{\sigma}_{u u}^{\prime}, \boldsymbol{\sigma}_{e e}^{\prime}, \boldsymbol{\sigma}_{x x}^{\prime}\right)^{\prime}$. Levando em conta as expressões de $\overline{\boldsymbol{d}}$. e $\mathrm{V}$ calculamos

$$
\begin{gathered}
\boldsymbol{d}_{\mu_{x i}}=-\left(0, \ldots, \beta_{i}, \ldots, 0,0, \ldots, 1, \ldots, 0\right)^{\prime}, \\
\boldsymbol{d}_{\alpha_{i}}=-\left(0, \ldots, 1, \ldots, 0,0^{\prime}\right)^{\prime}, \quad \boldsymbol{d}_{\beta_{i}}=-\left(0, \ldots, \mu_{x i}, \ldots, 0,0^{\prime}\right)^{\prime}, \\
\mathrm{V}_{\beta_{i}}=\left(\begin{array}{cc}
2 \mathrm{D}\left(0, \ldots, \beta_{i} \sigma_{x x i}, \ldots, 0\right) & \mathrm{D}\left(0, \ldots, \sigma_{x x i}, \ldots, 0\right) \\
0
\end{array}\right), \\
\mathrm{V}_{\sigma_{u u i}}=\left(\begin{array}{cc}
0 & 0 \\
& \mathrm{D}(0, \ldots, 1, \ldots, 0)
\end{array}\right), \mathrm{V}_{\sigma_{e e i}}=\left(\begin{array}{cc}
\mathrm{D}(0, \ldots, 1, \ldots, 0) & 0 \\
& 0
\end{array}\right)
\end{gathered}
$$

e

$$
\mathbf{V}_{\sigma_{x x i}}=\left(\begin{array}{cc}
\mathbf{D}\left(0, \ldots, \beta_{i}^{2}, \ldots, 0\right) & \mathbf{D}\left(0, \ldots, \beta_{i}, \ldots, 0\right) \\
& \mathbf{D}(0, \ldots, 1, \ldots, 0)
\end{array}\right),
$$

$i=1, \ldots, k$. Partindo de (4.6) e omitindo várias passagens chegamos a

$$
\frac{\partial}{\partial \mu_{x i}} \log \mathcal{L}=\frac{n}{\delta_{i}}\left[\beta_{i} \sigma_{u u i}\left(\bar{Y}_{i .}-\alpha_{i}-\beta_{i} \mu_{x i}\right)+\sigma_{e e i}\left(\bar{X}_{i .}-\mu_{x i}\right)\right],
$$




$$
\begin{gathered}
\frac{\partial}{\partial \alpha_{i}} \log \mathcal{L}=-\frac{n}{\delta_{i}}\left[\left(\sigma_{u u i}+\sigma_{x x i}\right)\left(\bar{Y}_{i .}-\alpha_{i}-\beta_{i} \mu_{x i}\right)+\beta_{i} \sigma_{x x i}\left(\bar{X}_{i .}-\mu_{x i}\right)\right], \\
\frac{\partial}{\partial \beta_{i}} \log \mathcal{L}=n \sigma_{x x i}\left(\beta_{i} p_{11 i}+p_{12 i}\right)+\mu_{x i} \frac{\partial}{\partial \alpha_{i}} \log \mathcal{L} \\
\frac{\partial}{\partial \sigma_{u u i}} \log \mathcal{L}=\frac{n}{2} p_{22 i} \quad, \quad \frac{\partial}{\partial \sigma_{e e i}} \log \mathcal{L}=\frac{n}{2} p_{11 i}
\end{gathered}
$$

$\mathrm{e}$

$i=1, \ldots, k$, onde

$$
\frac{\partial}{\partial \sigma_{x x i}} \log \mathcal{L}=\frac{n}{2}\left(\beta_{i}^{2} p_{11 i}+2 \beta_{i} p_{12 i}+p_{22 i}\right)
$$

$$
\begin{gathered}
p_{11 i}=h_{11 i}-\left(\sigma_{u u i}+\sigma_{x x i}\right) / \delta_{i}, \\
h_{11 i}=\left[\left(\sigma_{u u i}+\sigma_{x x i}\right)^{2} m_{Y Y i}^{*}-2 \beta_{i} \sigma_{x x i}\left(\sigma_{u u i}+\sigma_{x x i}\right) m_{Y X i}^{*}+\beta_{i}^{2} \sigma_{x x i}^{2} m_{X X i}^{*}\right] / \delta_{i}^{2}, \\
p_{12 i}=h_{12 i}+\beta_{i} \sigma_{x x i} / \delta_{i}, \\
h_{12 i}=\left\{-\left(\sigma_{u u i}+\sigma_{x x i}\right) \beta_{i} \sigma_{x x i} m_{Y Y i}^{*}+\left[\beta_{i}^{2} \sigma_{x x i}^{2}+\left(\sigma_{u u i}+\sigma_{x x i}\right)\left(\sigma_{e e i}+\beta_{i}^{2} \sigma_{x x i}\right)\right] m_{Y X i}^{*}\right. \\
\left.-\beta_{i} \sigma_{x x i}\left(\sigma_{e e i}+\beta_{i}^{2} \sigma_{x x i}\right) m_{X X i}^{*}\right\} / \delta_{i}^{2}, \\
p_{22 i}=h_{22 i}-\left(\sigma_{e e i}+\beta_{i}^{2} \sigma_{x x i}\right) / \delta_{i}, \\
h_{22 i}=\left[\beta_{i}^{2} \sigma_{x x i}^{2} m_{Y Y i}^{*}-2 \beta_{i} \sigma_{x x i}\left(\sigma_{e e i}+\beta_{i}^{2} \sigma_{x x i}\right) m_{Y X i}^{*}+\left(\sigma_{e e i}+\beta_{i}^{2} \sigma_{x x i}\right) m_{X X i}^{*}\right] / \delta_{i}^{2},
\end{gathered}
$$

com $m_{Y X i}^{*}=\sum_{j=1}^{n}\left(Y_{i, j}-\alpha_{i}-\beta_{i} \mu_{x i}\right) / n, i=1, \ldots, k$. Igualando o vetor gradiente ao vetor 0 obtemos um sistema de $p$ equações envolvendo os parâmetros. Em nossa avaliação, apenas no modelo 33 é possível obter expressões analíticas para os estimadores de $\theta$. Neste modelo, $\mathbf{V}^{-1}=\Sigma^{-1} \otimes \mathbf{I}_{k}$, com

$$
\Sigma^{-1}=\frac{1}{\delta}\left(\begin{array}{cc}
\sigma_{u u}+\sigma_{x x} & -\beta \sigma_{x x} \\
& \beta^{2} \sigma_{x x}+\sigma_{e e}
\end{array}\right)
$$

e $\delta=\left(\beta^{2} \sigma_{u u}+\sigma_{e e}\right) \sigma_{x x}+\sigma_{u u} \sigma_{e e}$, fato simplificador explorado por Dolby (1976) e que não acontece nos demais modelos da Tabela 4.2. Por exemplo, no modelo 58 a equação $\frac{\partial}{\partial \sigma_{u u}} \log \mathcal{L}=0$ fornece

$$
\begin{gathered}
\sum_{i=1}^{k} \frac{1}{\delta_{i}^{2}}\left[\left(\sigma_{u u}+\sigma_{x x i}\right)^{2} m_{Y X i}^{*}-2 \beta_{i} \sigma_{x x i}\left(\sigma_{u u}+\sigma_{x x i}\right) m_{Y X i}^{*}\right. \\
\left.+\beta_{i}^{2} \sigma_{x x i}^{2} m_{X X i}^{*}\right]=\sum_{i=1}^{k} \frac{1}{\delta_{i}}\left(\sigma_{u u}+\sigma_{x x i}\right)
\end{gathered}
$$


onde $\delta_{i}=\left(\beta_{i}^{2} \sigma_{u u}+\sigma_{e e}\right) \sigma_{x x i}+\sigma_{u u} \sigma_{e e}, i=1, \ldots, k$. O problema é semelhante à estimação por MV em modelos de componentes de variância desbalanceados (Searle, 1997, p. 462). Sendo assim, utilizaremos métodos numéricos para determinação dos pontos de máximo (local) de $\log \mathcal{L}(\boldsymbol{\theta} ; \mathbf{Y}, \mathbf{X})$. Os métodos de Newton e do escore de Fisher requerem as matrizes de informação observada e esperada, calculadas a seguir.

\subsubsection{Derivadas de segunda ordem}

Recordando que $\theta_{r}$ e $\theta_{s}$ são elementos genéricos de $\theta$ e designando por $m$ um elemento qualquer de $\mathbf{M}$, definimos $\boldsymbol{d}_{\theta_{s}, \theta_{r}}$ o vetor $2 k \times 1$ dado por $\frac{\partial}{\partial \theta_{s}} \boldsymbol{d}_{\theta_{r}}, \mathbf{M}_{\theta_{r}}$ a matriz $2 k \times 2 k$ formada pelos $\frac{\partial}{\partial \theta_{r}} m$ e $\mathbf{V}_{\theta_{s}, \theta_{r}}$ a matriz $2 k \times 2 k$ dada por $\frac{\partial}{\partial \theta_{s}} \mathbf{V}_{\theta_{r}}$. Recorrendo a Dolby (1976) segue de (4.6) que

$$
\begin{aligned}
& \frac{\partial^{2}}{\partial \theta_{s} \partial \theta_{r}} \log \mathcal{L}(\boldsymbol{\theta} ; \boldsymbol{Y}, \boldsymbol{X})=n\left[\frac { 1 } { 2 } \operatorname { t r } \left(-\mathbf{V}^{-1} \mathbf{V}_{\theta_{s}} \mathbf{V}^{-1} \mathbf{M ~ V}^{-1} \mathbf{V}_{\theta_{r}}+\mathbf{V}^{-1} \mathbf{M}_{\theta_{s}} \mathbf{V}^{-1} \mathbf{V}_{\theta_{r}}\right.\right. \\
& -\mathrm{V}^{-1} \mathrm{M} \mathrm{V}^{-1} \mathrm{~V}_{\theta_{s}} \mathrm{~V}^{-1} \mathrm{~V}_{\theta_{r}}+\mathrm{V}^{-1} \mathrm{MV}^{-1} \mathrm{~V}_{\theta_{s}, \theta_{r}} \\
& \left.+\mathrm{V}^{-1} \mathrm{~V}_{\theta_{s}} \mathrm{~V}^{-1} \mathrm{~V}_{\theta_{r}}-\mathrm{V}^{-1} \mathrm{~V}_{\theta_{s}, \theta_{r}}\right)-d_{\theta_{s}, \theta_{r}}^{\prime} \mathrm{V}^{-1} \bar{d} \text {. } \\
& \left.+\boldsymbol{d}_{\theta_{r}}^{\prime} \mathrm{V}^{-1} \mathrm{~V}_{\theta_{s}} \mathrm{~V}^{-1} \bar{d}-\boldsymbol{d}_{\theta_{r}}^{\prime} \mathrm{V}^{-1} \boldsymbol{d}_{\theta_{s}}\right]
\end{aligned}
$$


Assim como no cálculo do vetor gradiente, dispomos aqui de uma ferramenta geral para a obtenção da matriz hessiana de $\log \mathcal{L}$. Prosseguindo, decorre de (4.7) que

$$
\begin{aligned}
& \frac{\partial^{2}}{\partial \boldsymbol{\sigma}_{u u} \partial \boldsymbol{\beta}^{\prime}} \log \mathcal{L} \\
& =\left(\begin{array}{ccc}
\frac{\partial^{2}}{\partial \sigma_{u u 1} \partial \beta_{1}} \log \mathcal{L} & \cdots & 0 \\
& \ddots & \vdots \\
& & \frac{\partial^{2}}{\partial \sigma_{u u k} \partial \beta_{k}} \log \mathcal{L}
\end{array}\right) \\
& =\left(\begin{array}{ccc}
\frac{\partial^{2}}{\partial \sigma_{u u 1} \partial \beta_{1}} g_{1}\left(\varphi_{1}\right) & \cdots & 0 \\
+\sum_{j=1}^{n} \frac{\partial^{2}}{\partial \sigma_{u u 1} \partial \beta_{1}} g_{2}\left(\varphi_{1}, Y_{1 j}, X_{1 j}\right) & & \vdots \\
& \ddots & \\
& & +\sum_{j=1}^{n} \frac{\partial^{2}}{\partial \sigma_{u k k} \partial \beta_{k}} g_{1}\left(\varphi_{k}\right) \\
& &
\end{array}\right. \\
& =\left(\begin{array}{ccc}
b_{1} & \cdots & 0 \\
& \ddots & \vdots \\
& & b_{k}
\end{array}\right) \text {. }
\end{aligned}
$$

Se tivermos $\beta_{1}=\cdots=\beta_{k}=\beta$, então $\frac{\partial^{2}}{\partial \boldsymbol{\sigma}_{u u} \partial \beta} \log \mathcal{L}$ será um vetor $k \times 1$ dado por

$$
\begin{aligned}
\frac{\partial^{2}}{\partial \boldsymbol{\sigma}_{u u} \partial \beta} \log \mathcal{L} & =\left(\begin{array}{c}
\frac{\partial^{2}}{\partial \sigma_{u u 1} \partial \beta} g_{1}\left(\boldsymbol{\varphi}_{1}\right)+\sum_{j=1}^{n} \frac{\partial^{2}}{\partial \sigma_{u u 1} \partial \beta} g_{2}\left(\varphi_{1}, Y_{1 j}, X_{1 j}\right) \\
\vdots \\
\frac{\partial^{2}}{\partial \sigma_{u u k} \partial \beta} g_{1}\left(\varphi_{k}\right)+\sum_{j=1}^{n} \frac{\partial^{2}}{\partial \sigma_{u u k} \partial \beta} g_{2}\left(\varphi_{k}, Y_{k j}, X_{k j}\right)
\end{array}\right) \\
& =\left(\begin{array}{c}
b_{1} \\
\vdots \\
b_{k}
\end{array}\right)_{\mid \beta_{1}=\cdots=\beta_{k}=\beta} .
\end{aligned}
$$

Se tivermos $\beta_{1}=\cdots=\beta_{k}=\beta$ e $\sigma_{u u 1}=\cdots=\sigma_{u u k}=\sigma_{u u}$, então $\frac{\partial^{2}}{\partial \sigma \partial \beta} \log \mathcal{L}$ será um escalar expresso por

$$
\begin{aligned}
\frac{\partial^{2}}{\partial \sigma_{u u} \partial \beta} \log \mathcal{L} & =\sum_{i=1}^{k} \frac{\partial^{2}}{\partial \sigma_{u u} \partial \beta} g_{1}\left(\boldsymbol{\varphi}_{i}\right)+\sum_{i=1}^{k} \sum_{j=1}^{n} \frac{\partial^{2}}{\partial \sigma_{u u} \partial \beta} g_{2}\left(\boldsymbol{\varphi}_{i}, Y_{i j}, X_{i j}\right) \\
& =\left(b_{1}+\cdots+b_{k}\right) \mid \begin{array}{c}
\beta_{1}=\cdots=\beta_{k}=\beta, \\
\sigma_{u u 1}=\cdots=\sigma_{u u k}=\sigma_{u u}
\end{array}
\end{aligned} .
$$

No modelo 64 os elementos em (4.9) são organizados em uma matriz $6 k \times 6 k$. Em um modelo qualquer a matriz de derivadas de segunda ordem, com dimensão $p \times p$, 
tem expressão

$$
\Lambda \frac{\partial^{2}}{\partial \theta \partial \theta^{\prime}} \log \mathcal{L}(\boldsymbol{\theta} ; \mathbf{Y}, \mathbf{X}) \Lambda^{\prime}
$$

onde $\boldsymbol{\Lambda}$ foi definida em (4.8).

Passamos ao cálculo das derivadas em (4.9), iniciando com

$$
\begin{gathered}
\boldsymbol{d}_{\beta_{i}, \mu_{x i}}=d_{\mu_{x i}, \beta_{i}}=-\left(0, \ldots, 1, \ldots, 0,0^{\prime}\right)^{\prime}, \\
\mathbf{V}_{\beta_{i}, \beta_{i}}=\left(\begin{array}{rr}
2 \mathbf{D}\left(0, \ldots, \sigma_{x x i}, \ldots, 0\right) & \mathbf{0} \\
0
\end{array}\right), \\
\mathbf{V}_{\sigma_{x x i}, \beta_{i}}=\mathrm{V}_{\beta_{i}, \sigma_{x x i}}=\left(\begin{array}{cc}
2 \mathbf{D}\left(0, \ldots, \beta_{i}, \ldots, 0\right) & \mathbf{D}(0, \ldots, 1, \ldots, 0) \\
0
\end{array}\right),
\end{gathered}
$$

$i=1, \ldots, k$, sendo que $\boldsymbol{d}_{\theta_{s}, \theta_{r}}$ e $\mathrm{V}_{\theta_{s}, \theta_{r}}$ restantes são nulas, e $\mathbf{M}_{\theta_{\boldsymbol{r}}}=\boldsymbol{d}_{\theta_{\boldsymbol{r}}} \overline{\boldsymbol{d}}^{\prime}+\overline{\boldsymbol{d}}^{\prime} \boldsymbol{d}_{\theta_{r}}$, $\theta_{r} \in \theta$. Extensas operações algébricas conduzem a

$$
\begin{gathered}
\frac{\partial^{2}}{\partial \boldsymbol{\mu}_{x} \partial \boldsymbol{\mu}_{x}^{\prime}} \log \mathcal{L}=-n \mathbf{D}\left(\left(\boldsymbol{\beta} \odot \boldsymbol{\beta} \odot \boldsymbol{\sigma}_{u u}+\boldsymbol{\sigma}_{e e}\right) \oslash \boldsymbol{\delta}\right) \\
\frac{\partial^{2}}{\partial \boldsymbol{\alpha} \partial \boldsymbol{\mu}_{x}^{\prime}} \log \mathcal{L}=-n \mathbf{D}\left(\boldsymbol{\beta} \odot \boldsymbol{\sigma}_{u u} \oslash \boldsymbol{\delta}\right) \\
\frac{\partial^{2}}{\partial \boldsymbol{\beta} \partial \boldsymbol{\mu}_{x}^{\prime}} \log \mathcal{L}=n \mathbf{D}(\boldsymbol{v})
\end{gathered}
$$

onde

$$
\begin{aligned}
\boldsymbol{v}= & \frac{1}{n} \frac{\partial}{\partial \boldsymbol{\alpha}} \log \mathcal{L}-\boldsymbol{\sigma}_{x x} \odot\left\{\left(2 \boldsymbol{\beta} \odot \boldsymbol{\beta} \odot \boldsymbol{\sigma}_{u u}+\boldsymbol{\sigma}_{e e}\right) \odot\left[\left(\boldsymbol{\sigma}_{u u}+\boldsymbol{\sigma}_{x x}\right) \odot \overline{\boldsymbol{d}}_{. Y}-\boldsymbol{\beta} \odot \boldsymbol{\sigma}_{x x} \odot \overline{\boldsymbol{d}}_{. X}\right]\right. \\
& \left.+\boldsymbol{\beta} \odot \boldsymbol{\sigma}_{u u} \odot\left[-\boldsymbol{\beta} \odot \boldsymbol{\sigma}_{x \boldsymbol{x}} \odot \overline{\boldsymbol{d}}_{. Y}+\left(\boldsymbol{\beta} \odot \boldsymbol{\beta} \odot \boldsymbol{\sigma}_{x x}+\boldsymbol{\sigma}_{e e}\right) \odot \overline{\boldsymbol{d}}_{. X}\right]\right\}-\boldsymbol{\mu}_{x} \odot \boldsymbol{\beta} \odot \boldsymbol{\sigma}_{u u} \oslash \boldsymbol{\delta}
\end{aligned}
$$

com $\bar{d}_{. Y}$ e $\bar{d}_{\cdot X}$ obtidos de $\bar{d} .=\left(\bar{d}_{. Y}^{\prime}, \bar{d}_{. X}^{\prime}\right)^{\prime}$

$$
\frac{\partial^{2}}{\partial \boldsymbol{\sigma}_{u u} \partial \boldsymbol{\mu}_{x}^{\prime}} \log \mathcal{L}=-n \mathbf{D}(\boldsymbol{v}),
$$

onde

$$
\begin{gathered}
\boldsymbol{v}=\boldsymbol{\sigma}_{e e} \odot\left[-\boldsymbol{\beta} \odot \boldsymbol{\sigma}_{x x} \odot \overline{\boldsymbol{d}}_{. Y}+\left(\boldsymbol{\beta} \odot \boldsymbol{\beta} \odot \boldsymbol{\sigma}_{x x}+\boldsymbol{\sigma}_{e e}\right) \odot \overline{\boldsymbol{d}}_{. X}\right] \oslash(\boldsymbol{\delta} \odot \boldsymbol{\delta}), \\
\frac{\partial^{2}}{\partial \boldsymbol{\sigma}_{e e} \partial \boldsymbol{\mu}_{x}^{\prime}} \log \mathcal{L}=-n \mathrm{D}(\boldsymbol{v}),
\end{gathered}
$$


onde

$$
\begin{gathered}
\boldsymbol{v}=\boldsymbol{\beta} \odot \boldsymbol{\sigma}_{u u} \odot\left[\left(\boldsymbol{\sigma}_{u u}+\boldsymbol{\sigma}_{x x}\right) \odot \overline{\boldsymbol{d}}_{. Y}-\boldsymbol{\beta} \odot \boldsymbol{\sigma}_{x x} \odot \overline{\boldsymbol{d}}_{. X}\right] \oslash(\boldsymbol{\delta} \odot \boldsymbol{\delta}), \\
\frac{\partial^{2}}{\partial \boldsymbol{\sigma}_{x x} \partial \boldsymbol{\mu}_{x}^{\prime}} \log \mathcal{L}=-n \mathrm{D}(\boldsymbol{v}),
\end{gathered}
$$

onde

$$
\begin{gathered}
\boldsymbol{v}=\left(\boldsymbol{\beta} \odot \boldsymbol{\beta} \odot \boldsymbol{\sigma}_{u u}+\boldsymbol{\sigma}_{e e}\right) \odot\left(\boldsymbol{\beta} \odot \boldsymbol{\sigma}_{u u} \odot \overline{\boldsymbol{d}}_{. Y}+\boldsymbol{\sigma}_{e e} \odot \overline{\boldsymbol{d}}_{. X}\right) \oslash(\boldsymbol{\delta} \odot \boldsymbol{\delta}), \\
\frac{\partial^{2}}{\partial \boldsymbol{\alpha} \partial \boldsymbol{\alpha}^{\prime}} \log \mathcal{L}=-n \mathbf{D}\left(\left(\boldsymbol{\sigma}_{u u}+\boldsymbol{\sigma}_{x x}\right) \oslash \boldsymbol{\delta}\right) \\
\frac{\partial^{2}}{\partial \boldsymbol{\beta} \partial \boldsymbol{\alpha}^{\prime}} \log \mathcal{L}=n \mathrm{D}(\boldsymbol{v})
\end{gathered}
$$

onde

$$
\begin{gathered}
\boldsymbol{v}=-\boldsymbol{\sigma}_{x x} \odot\left\{2 \odot \boldsymbol{\beta} \odot \boldsymbol{\sigma}_{u u} \odot\left(\boldsymbol{\sigma}_{u u}+\boldsymbol{\sigma}_{x x}\right) \odot \overline{\boldsymbol{d}}_{. Y}-\left[\boldsymbol{\beta} \odot \boldsymbol{\beta} \odot \boldsymbol{\sigma}_{u u} \odot \boldsymbol{\sigma}_{x x}\right.\right. \\
\left.\left.-\boldsymbol{\sigma}_{e e} \odot\left(\boldsymbol{\sigma}_{u u}+\boldsymbol{\sigma}_{x x}\right)\right] \odot \overline{\boldsymbol{d}}_{. X}\right\} \oslash(\boldsymbol{\delta} \odot \boldsymbol{\delta})-\boldsymbol{\mu}_{x} \odot\left(\boldsymbol{\sigma}_{u u}+\boldsymbol{\sigma}_{x x}\right) \oslash \boldsymbol{\delta} \\
\frac{\partial^{2}}{\partial \boldsymbol{\sigma}_{u u} \partial \boldsymbol{\alpha}^{\prime}} \log \mathcal{L}=n \mathbf{D}(\boldsymbol{v})
\end{gathered}
$$

onde

$$
\begin{gathered}
\boldsymbol{v}=\boldsymbol{\beta} \odot \boldsymbol{\sigma}_{x x} \odot\left[-\boldsymbol{\beta} \odot \boldsymbol{\sigma}_{x x} \odot \overline{\boldsymbol{d}}_{. Y}+\left(\boldsymbol{\beta} \odot \boldsymbol{\beta} \odot \boldsymbol{\sigma}_{x x}+\boldsymbol{\sigma}_{e e}\right) \odot \overline{\boldsymbol{d}}_{. X}\right] \oslash(\boldsymbol{\delta} \odot \boldsymbol{\delta}), \\
\frac{\partial^{2}}{\partial \boldsymbol{\sigma}_{e e} \partial \boldsymbol{\alpha}^{\prime}} \log \mathcal{L}=-n \mathbf{D}(\boldsymbol{v})
\end{gathered}
$$

onde

$$
\begin{gathered}
\boldsymbol{v}=\left(\boldsymbol{\sigma}_{u u}+\boldsymbol{\sigma}_{x x}\right) \odot\left[\left(\boldsymbol{\sigma}_{u u}+\boldsymbol{\sigma}_{x x}\right) \odot \overline{\boldsymbol{d}}_{. Y}-\boldsymbol{\beta} \odot \boldsymbol{\sigma}_{x x} \odot \overline{\boldsymbol{d}}_{. X}\right] \oslash(\boldsymbol{\delta} \odot \boldsymbol{\delta}), \\
\frac{\partial^{2}}{\partial \boldsymbol{\sigma}_{x x} \partial \boldsymbol{\alpha}^{\prime}} \log \mathcal{L}=-n \mathbf{D}(\boldsymbol{v})
\end{gathered}
$$

onde

$$
\begin{gathered}
\boldsymbol{v}=\boldsymbol{\beta} \odot \boldsymbol{\sigma}_{u u} \odot\left(\boldsymbol{\beta} \odot \boldsymbol{\sigma}_{u u} \odot \overline{\boldsymbol{d}}_{. Y}+\boldsymbol{\sigma}_{e e} \odot \overline{\boldsymbol{d}}_{. X}\right) \oslash(\boldsymbol{\delta} \odot \delta), \\
\frac{\partial^{2}}{\partial \boldsymbol{\beta} \partial \boldsymbol{\beta}^{\prime}} \log \mathcal{L}=n \mathrm{D}(\boldsymbol{v}),
\end{gathered}
$$


onde

$$
\begin{aligned}
\boldsymbol{v}= & -\boldsymbol{\sigma}_{x x} \odot \boldsymbol{\sigma}_{x x} \odot\left[\left(4 \boldsymbol{\beta} \odot \boldsymbol{\beta} \odot \boldsymbol{\sigma}_{u u}+\boldsymbol{\beta} \odot \boldsymbol{\beta} \odot \boldsymbol{\sigma}_{x x}+\boldsymbol{\sigma}_{e e}\right) \odot \boldsymbol{h}_{11}\right. \\
& \left.+2 \boldsymbol{\beta} \odot\left(2 \boldsymbol{\sigma}_{u u}+\boldsymbol{\sigma}_{x x}\right) \odot \boldsymbol{h}_{12}+\left(\boldsymbol{\sigma}_{u u}+\boldsymbol{\sigma}_{x x}\right) \odot \boldsymbol{h}_{22}\right] \oslash \boldsymbol{\delta}-\boldsymbol{\mu}_{x} \odot \boldsymbol{\beta} \odot \boldsymbol{\sigma}_{u u} \odot \boldsymbol{\sigma}_{x x} \\
& \odot\left[2\left(\boldsymbol{\sigma}_{u u}+\boldsymbol{\sigma}_{x x}\right) \odot \overline{\boldsymbol{d}}_{. Y}-\boldsymbol{\beta} \odot \boldsymbol{\sigma}_{x x} \odot \overline{\boldsymbol{d}}_{. X}\right] \oslash(\boldsymbol{\delta} \odot \boldsymbol{\delta})+\boldsymbol{p}_{\boldsymbol{1 1}} \odot \boldsymbol{\sigma}_{x x}+\boldsymbol{\sigma}_{x x} \odot \boldsymbol{\sigma}_{x x} \\
& \odot\left(2 \boldsymbol{\beta} \odot \boldsymbol{\beta} \odot \boldsymbol{\sigma}_{u u} \odot \boldsymbol{\sigma}_{u u}+\boldsymbol{\delta}\right) \oslash(\boldsymbol{\delta} \odot \boldsymbol{\delta})-\boldsymbol{\mu}_{x} \odot \boldsymbol{\sigma}_{x x} \odot\left\{2 \boldsymbol{\beta} \odot \boldsymbol{\sigma}_{u u} \odot\left(\boldsymbol{\sigma}_{u u}+\boldsymbol{\sigma}_{x x}\right) \odot \overline{\boldsymbol{d}}_{x}\right. \\
& \left.-\left[\boldsymbol{\beta} \odot \boldsymbol{\beta} \odot \boldsymbol{\sigma}_{u u} \odot \boldsymbol{\sigma}_{x x}-\boldsymbol{\sigma}_{e e} \odot\left(\boldsymbol{\sigma}_{u u}+\boldsymbol{\sigma}_{x x}\right)\right] \odot \overline{\boldsymbol{d}}_{. X}\right\} \oslash(\boldsymbol{\delta} \odot \boldsymbol{\delta}) \\
& -\boldsymbol{\mu}_{x} \odot \boldsymbol{\mu}_{x} \odot\left(\boldsymbol{\sigma}_{u u}+\boldsymbol{\sigma}_{x x}\right) \oslash \boldsymbol{\delta}
\end{aligned}
$$

com $h_{11}$ e $p_{11}$ tendo elementos $h_{11 i}$ e $p_{11 i}$, respectivamente, definidos na p. 68 ,

$$
\frac{\partial^{2}}{\partial \boldsymbol{\sigma}_{u u} \partial \boldsymbol{\beta}^{\prime}} \log \mathcal{L}=n \mathbf{D}(\boldsymbol{v})
$$

onde

$$
\begin{gathered}
\boldsymbol{v}=-\boldsymbol{\sigma}_{x x} \odot\left[\left(-\boldsymbol{\beta} \odot \boldsymbol{\beta} \odot \boldsymbol{\sigma}_{x x}+\boldsymbol{\sigma}_{e e}\right) \odot \boldsymbol{h}_{12}-\boldsymbol{\beta} \odot \boldsymbol{\sigma}_{x x} \odot \boldsymbol{h}_{22}\right] \oslash \boldsymbol{\delta} \\
-\boldsymbol{\beta} \odot \boldsymbol{\sigma}_{e e} \odot \boldsymbol{\sigma}_{x x} \odot \boldsymbol{\sigma}_{x x} \oslash(\boldsymbol{\delta} \odot \boldsymbol{\delta})+\boldsymbol{\mu}_{x} \odot \boldsymbol{\beta} \odot \boldsymbol{\sigma}_{x x} \\
\odot\left[-\boldsymbol{\beta} \odot \boldsymbol{\sigma}_{x x} \odot \overline{\boldsymbol{d}}_{. Y}+\left(\boldsymbol{\beta} \odot \boldsymbol{\beta} \odot \boldsymbol{\sigma}_{x x}+\boldsymbol{\sigma}_{e e}\right) \odot \overline{\boldsymbol{d}}_{. X}\right] \oslash(\boldsymbol{\delta} \odot \boldsymbol{\delta}) \\
\frac{\partial^{2}}{\partial \boldsymbol{\sigma}_{e e} \partial \boldsymbol{\beta}^{\prime}} \log \mathcal{L}=n \mathrm{D}(\boldsymbol{v})
\end{gathered}
$$

onde

$$
\begin{gathered}
\boldsymbol{v}=-\boldsymbol{\sigma}_{x x} \odot\left[\boldsymbol{\beta} \odot\left(2 \boldsymbol{\sigma}_{u u}+\boldsymbol{\sigma}_{x x}\right) \odot \boldsymbol{h}_{11}+\left(\boldsymbol{\sigma}_{u u}+\boldsymbol{\sigma}_{x x}\right) \odot \boldsymbol{h}_{12}\right] \oslash \boldsymbol{\delta}+\boldsymbol{\beta} \odot \boldsymbol{\sigma}_{u u} \odot \boldsymbol{\sigma}_{x x} \odot\left(\boldsymbol{\sigma}_{u u}+\boldsymbol{\sigma}_{x x}\right) \\
-\boldsymbol{\mu}_{x} \odot\left(\boldsymbol{\sigma}_{u u}+\boldsymbol{\sigma}_{x x}\right) \odot\left[\left(\boldsymbol{\sigma}_{u u}+\boldsymbol{\sigma}_{x x}\right) \odot \overline{\boldsymbol{d}}_{. Y}-\boldsymbol{\beta} \odot \boldsymbol{\sigma}_{x x} \odot \overline{\boldsymbol{d}}_{. X}\right] \oslash(\boldsymbol{\delta} \odot \boldsymbol{\delta}), \\
\frac{\partial^{2}}{\partial \boldsymbol{\sigma}_{x x} \partial \boldsymbol{\beta}^{\prime}} \log \mathcal{L}=n \mathbf{D}(v),
\end{gathered}
$$

onde

$$
\begin{aligned}
\boldsymbol{v}= & -\boldsymbol{\sigma}_{x x} \odot\left[\boldsymbol{\beta} \odot\left(2 \boldsymbol{\beta} \odot \boldsymbol{\beta} \odot \boldsymbol{\sigma}_{u u}+\boldsymbol{\sigma}_{e e}\right) \odot \boldsymbol{h}_{11}+\left(3 \boldsymbol{\beta} \odot \boldsymbol{\beta} \odot \boldsymbol{\sigma}_{u u}+\boldsymbol{\sigma}_{e e}\right) \odot \boldsymbol{h}_{12}\right. \\
& \left.+\boldsymbol{\beta} \odot \boldsymbol{\sigma}_{u u} \odot \boldsymbol{h}_{22}\right] \oslash \boldsymbol{\delta}+\boldsymbol{\beta} \odot \boldsymbol{p}_{11}+\boldsymbol{p}_{12}+\boldsymbol{\beta} \odot \boldsymbol{\sigma}_{u u} \odot \boldsymbol{\sigma}_{x x} \\
& \odot\left(\boldsymbol{\beta} \odot \boldsymbol{\beta} \odot \boldsymbol{\sigma}_{u u}+\boldsymbol{\sigma}_{e e}\right) \oslash(\boldsymbol{\delta} \odot \boldsymbol{\delta})-\boldsymbol{\mu}_{x} \odot \boldsymbol{\beta} \odot \boldsymbol{\sigma}_{u u} \\
& \odot\left(\boldsymbol{\beta} \odot \boldsymbol{\sigma}_{u u} \odot \overline{\boldsymbol{d}}_{. Y}+\boldsymbol{\sigma}_{e e} \odot \overline{\boldsymbol{d}}_{. X}\right) \oslash(\boldsymbol{\delta} \odot \boldsymbol{\delta})
\end{aligned}
$$




$$
\frac{\partial^{2}}{\partial \boldsymbol{\sigma}_{u u} \partial \boldsymbol{\sigma}_{u u}^{\prime}} \log \mathcal{L}=n \mathbf{D}(\boldsymbol{v})
$$

onde

$$
\begin{gathered}
\boldsymbol{v}=-\left(\boldsymbol{\beta} \odot \boldsymbol{\beta} \odot \boldsymbol{\sigma}_{x x}+\boldsymbol{\sigma}_{e e}\right) \odot \boldsymbol{h}_{22} \oslash \boldsymbol{\delta}+\left(\boldsymbol{\sigma}_{e e}+\boldsymbol{\beta} \odot \boldsymbol{\beta} \odot \boldsymbol{\sigma}_{x x}\right) \\
\odot\left(\boldsymbol{\sigma}_{e e}+\boldsymbol{\beta} \odot \boldsymbol{\beta} \odot \boldsymbol{\sigma}_{x x}\right) \oslash(2 \boldsymbol{\delta} \odot \boldsymbol{\delta}) \\
\frac{\partial^{2}}{\partial \boldsymbol{\sigma}_{e e} \partial \boldsymbol{\sigma}_{u u}^{\prime}} \log \mathcal{L}=n \mathbf{D}(\boldsymbol{v})
\end{gathered}
$$

onde

$$
\begin{gathered}
\boldsymbol{v}=\boldsymbol{\beta} \odot \boldsymbol{\sigma}_{x x} \odot \boldsymbol{h}_{12} \oslash \boldsymbol{\delta}+\boldsymbol{\beta} \odot \boldsymbol{\beta} \odot \boldsymbol{\sigma}_{x x} \odot \boldsymbol{\sigma}_{x x} \oslash(2 \boldsymbol{\delta} \odot \boldsymbol{\delta}), \\
\frac{\partial^{2}}{\partial \boldsymbol{\sigma}_{x x} \partial \boldsymbol{\sigma}_{u u}^{\prime}} \log \mathcal{L}=n \mathbf{D}(\boldsymbol{v}),
\end{gathered}
$$

onde

$$
\begin{gathered}
\boldsymbol{v}=-\boldsymbol{\sigma}_{e e} \odot\left(\boldsymbol{\beta} \odot \boldsymbol{h}_{12}+\boldsymbol{h}_{22}\right) \oslash \boldsymbol{\delta}+\boldsymbol{\sigma}_{e e} \odot \boldsymbol{\sigma}_{e e} \oslash(2 \boldsymbol{\delta} \odot \boldsymbol{\delta}), \\
\frac{\partial^{2}}{\partial \boldsymbol{\sigma}_{e e} \partial \boldsymbol{\sigma}_{e e}^{\prime}} \log \mathcal{L}=n \mathbf{D}(\boldsymbol{v})
\end{gathered}
$$

onde

$$
\begin{gathered}
\boldsymbol{v}=-\left(\boldsymbol{\sigma}_{u u}+\boldsymbol{\sigma}_{x x}\right) \odot \boldsymbol{h}_{11} \oslash \boldsymbol{\delta}+\left(\boldsymbol{\sigma}_{u u}+\boldsymbol{\sigma}_{x x}\right) \odot\left(\boldsymbol{\sigma}_{u u}+\boldsymbol{\sigma}_{x x}\right) \oslash(2 \boldsymbol{\delta} \odot \boldsymbol{\delta}), \\
\frac{\partial^{2}}{\partial \boldsymbol{\sigma}_{e e} \partial \boldsymbol{\sigma}_{x x}^{\prime}} \log \mathcal{L}=n \mathbf{D}(\boldsymbol{v}),
\end{gathered}
$$

onde

$$
v=-\beta \odot \boldsymbol{\sigma}_{u u} \odot\left(\beta \odot \boldsymbol{h}_{11}+\boldsymbol{h}_{12}\right) \oslash \boldsymbol{\delta}+\boldsymbol{\beta} \odot \boldsymbol{\beta} \odot \boldsymbol{\sigma}_{u u} \odot \boldsymbol{\sigma}_{u u} \oslash(2 \boldsymbol{\delta} \odot \boldsymbol{\delta}),
$$

e, finalmente,

$$
\frac{\partial^{2}}{\partial \boldsymbol{\sigma}_{x x} \partial \sigma_{x x}^{\prime}} \log \mathcal{L}=n \mathbf{D}(\boldsymbol{v})
$$

onde

$$
\begin{aligned}
\boldsymbol{v}= & -\left(\boldsymbol{\beta} \odot \boldsymbol{\beta} \odot \boldsymbol{\sigma}_{u u}+\boldsymbol{\sigma}_{e e}\right) \odot\left(\boldsymbol{\beta} \odot \boldsymbol{\beta} \odot \boldsymbol{h}_{11}+2 \boldsymbol{\beta} \odot \boldsymbol{h}_{12}+\boldsymbol{h}_{22}\right) \oslash \boldsymbol{\delta} \\
& +\left(\boldsymbol{\beta} \odot \boldsymbol{\beta} \odot \boldsymbol{\sigma}_{u u}+\boldsymbol{\sigma}_{e e}\right) \odot\left(\boldsymbol{\beta} \odot \boldsymbol{\beta} \odot \boldsymbol{\sigma}_{u u}+\boldsymbol{\sigma}_{e e}\right) \oslash(2 \boldsymbol{\delta} \odot \boldsymbol{\delta})
\end{aligned}
$$

Juntando esses 21 blocos formamos a matriz $6 k \times 6 k \frac{\partial^{2}}{\partial \boldsymbol{\theta}_{\partial} \boldsymbol{\theta}^{\prime}} \log \mathcal{L}(\boldsymbol{\theta} ; \mathbf{Y}, \mathbf{X})$ correspondente ao modelo 64, a partir da qual se consegue a matriz de qualquer modelo da Tabela 4.2 através de (4.10). 
A matriz de informação esperada no modelo 64, denotada por $\mathcal{I}(\boldsymbol{\theta})$, segue de

$$
\mathcal{I}(\boldsymbol{\theta})=-\mathrm{E}\left(\frac{\partial^{2}}{\partial \boldsymbol{\theta} \partial \boldsymbol{\theta}^{\prime}} \log \mathcal{L}(\boldsymbol{\theta} ; \mathbf{Y}, \mathbf{X})\right)
$$

Tomando esperança de (4.9) ou usando a técnica de Dolby (1976) chega-se à expressão para um elemento genérico de $\mathcal{I}(\theta)$ :

$$
-\mathrm{E}\left(\frac{\partial^{2}}{\partial \theta_{s} \partial \theta_{r}} \log \mathcal{L}(\boldsymbol{\theta} ; \boldsymbol{Y}, \boldsymbol{X})\right)=n\left[\frac{1}{2} \operatorname{tr}\left(-\mathrm{V}^{-1} \mathbf{V}_{\theta_{s}} \mathbf{V}^{-1} \mathbf{V}_{\theta_{r}}\right)+\boldsymbol{d}_{\theta_{r}}^{\prime} \mathbf{V}^{-1} \boldsymbol{d}_{\theta_{s}}\right]
$$

$\operatorname{com} \theta_{s}, \theta_{r} \in \theta$. Efetuando estas operações resulta em $\mathcal{I}(\boldsymbol{\theta})=n \mathcal{I}_{1}(\theta)$,

$$
\begin{aligned}
& \mathcal{I}_{1}(\theta)=\left(\begin{array}{cccccc}
\mathcal{I}_{\mu_{x} \mu_{x}} & \mathcal{I}_{\mu_{x} \alpha} & \mathcal{I}_{\mu_{x} \beta} & 0 & 0 & 0 \\
& \mathcal{I}_{\alpha \alpha} & \mathcal{I}_{\alpha \beta} & 0 & 0 & 0 \\
& & \mathcal{I}_{\beta \beta} & \mathcal{I}_{\beta \sigma_{u u}} & \mathcal{I}_{\beta \sigma_{e e}} & \mathcal{I}_{\beta \sigma_{x x}} \\
& & & \mathcal{I}_{\sigma_{u u} \sigma_{u u}} & \mathcal{I}_{\sigma_{u u} \sigma_{e e}} & \mathcal{I}_{\sigma_{u u} \sigma_{x x}} \\
& & & & \mathcal{I}_{\sigma_{e e} \sigma_{e e}} & \mathcal{I}_{\sigma_{e e} \sigma_{x x}} \\
& & & & & \mathcal{I}_{\sigma_{x x} \sigma_{x x}}
\end{array}\right) \\
& \mathcal{I}_{\mu_{x} \mu_{x}}=\mathbf{D}\left(\left(\beta \odot \beta \odot \boldsymbol{\sigma}_{u u}+\boldsymbol{\sigma}_{e e}\right) \oslash \boldsymbol{\delta}\right) \quad, \quad \mathcal{I}_{\mu_{x} \alpha}=\mathbf{D}\left(\boldsymbol{\beta} \odot \boldsymbol{\sigma}_{u u} \oslash \boldsymbol{\delta}\right), \\
& \mathcal{I}_{\mu_{x} \beta}=\mathbf{D}\left(\boldsymbol{\mu}_{x} \odot \boldsymbol{\beta} \odot \boldsymbol{\sigma}_{u u} \oslash \boldsymbol{\delta}\right) \quad, \quad \mathcal{I}_{\alpha \alpha}=\mathbf{D}\left(\left(\boldsymbol{\sigma}_{u u}+\boldsymbol{\sigma}_{x x}\right) \oslash \boldsymbol{\delta}\right) \text {, } \\
& \mathcal{I}_{\alpha \beta}=\mathbf{D}\left(\boldsymbol{\mu}_{x} \odot\left(\boldsymbol{\sigma}_{u u}+\boldsymbol{\sigma}_{x x}\right) \oslash \delta\right) \quad, \quad \mathcal{I}_{\beta \beta}=\mathbf{D}(\boldsymbol{v}),
\end{aligned}
$$

onde

$$
\begin{gathered}
\boldsymbol{v}=\boldsymbol{\mu}_{x} \odot \boldsymbol{\mu}_{x} \odot\left(\boldsymbol{\sigma}_{u u}+\boldsymbol{\sigma}_{x x}\right) \oslash \boldsymbol{\delta}+\boldsymbol{\sigma}_{x x} \odot \boldsymbol{\sigma}_{x x} \odot\left(2 \boldsymbol{\beta} \odot \boldsymbol{\beta} \odot \boldsymbol{\sigma}_{u u} \odot \boldsymbol{\sigma}_{u u}+\boldsymbol{\delta}\right) \oslash(\boldsymbol{\delta} \odot \boldsymbol{\delta}), \\
\mathcal{I}_{\beta \sigma_{u u}}=-\mathrm{D}\left(\boldsymbol{\beta} \odot \boldsymbol{\sigma}_{x x} \odot \boldsymbol{\sigma}_{x x} \odot \boldsymbol{\sigma}_{e e} \oslash(\boldsymbol{\delta} \odot \boldsymbol{\delta})\right), \\
\mathcal{I}_{\beta \sigma_{e e}}=\mathrm{D}\left(\boldsymbol{\beta} \odot \boldsymbol{\sigma}_{u u} \odot \boldsymbol{\sigma}_{x x} \odot\left(\boldsymbol{\sigma}_{u u}+\boldsymbol{\sigma}_{x x}\right) \oslash(\boldsymbol{\delta} \odot \boldsymbol{\delta})\right), \\
\mathcal{I}_{\beta \sigma_{x x}}=\mathbf{D}\left(\boldsymbol{\beta} \odot \boldsymbol{\sigma}_{u u} \odot \boldsymbol{\sigma}_{x x} \odot\left(\boldsymbol{\beta} \odot \boldsymbol{\beta} \odot \boldsymbol{\sigma}_{u u}+\boldsymbol{\sigma}_{e e}\right) \oslash(\boldsymbol{\delta} \odot \boldsymbol{\delta})\right) \quad, \quad \mathcal{I}_{\sigma_{u u} \sigma_{u u}}=\frac{1}{2} \mathrm{D}(\boldsymbol{v}),
\end{gathered}
$$

onde

$$
\begin{gathered}
\boldsymbol{v}=\left(\boldsymbol{\beta} \odot \boldsymbol{\beta} \odot \boldsymbol{\sigma}_{x x}+\boldsymbol{\sigma}_{e e}\right) \odot\left(\boldsymbol{\beta} \odot \boldsymbol{\beta} \odot \boldsymbol{\sigma}_{x x}+\boldsymbol{\sigma}_{e e}\right) \oslash(\boldsymbol{\delta} \odot \boldsymbol{\delta}) \\
\mathcal{I}_{\sigma_{u u} \sigma_{e e}}=\frac{1}{2} \mathbf{D}\left(\boldsymbol{\beta} \odot \boldsymbol{\beta} \odot \boldsymbol{\sigma}_{x x} \odot \boldsymbol{\sigma}_{x x} \oslash(\boldsymbol{\delta} \odot \boldsymbol{\delta})\right), \quad \boldsymbol{I}_{\sigma_{u u} \sigma_{x x}}=\frac{1}{2} \mathbf{D}\left(\boldsymbol{\sigma}_{e e} \odot \boldsymbol{\sigma}_{e e} \oslash(\boldsymbol{\delta} \odot \boldsymbol{\delta})\right) \\
\mathcal{I}_{\sigma_{e e} \sigma_{e e}}=\frac{1}{2} \mathbf{D}\left(\left(\boldsymbol{\sigma}_{u u}+\boldsymbol{\sigma}_{x x}\right) \odot\left(\boldsymbol{\sigma}_{u u}+\boldsymbol{\sigma}_{x x}\right) \oslash(\boldsymbol{\delta} \odot \boldsymbol{\delta})\right)
\end{gathered}
$$




$$
\mathcal{I}_{\sigma_{e e} \sigma_{x x}}=\frac{1}{2} \mathbf{D}\left(\beta \odot \beta \odot \sigma_{u u} \odot \boldsymbol{\sigma}_{u u} \oslash(\delta \odot \delta)\right) \quad \text { e } \quad \mathcal{I}_{\sigma_{x x} \sigma_{x x}}=\frac{1}{2} \mathbf{D}(\boldsymbol{v}),
$$

onde

$$
\boldsymbol{v}=\left(\boldsymbol{\beta} \odot \boldsymbol{\beta} \odot \boldsymbol{\sigma}_{u u}\right) \odot\left(\boldsymbol{\beta} \odot \boldsymbol{\beta} \odot \boldsymbol{\sigma}_{u u}\right) \oslash(\boldsymbol{\delta} \odot \boldsymbol{\delta}) .
$$

A matriz $\mathcal{I}(\theta)$ é uma generalização da matriz $Z$ de Dolby $(1976$, p. 45). Em um modelo qualquer a matriz de informação esperada, com dimensão $p \times p$, é dada por

$$
\Lambda \mathcal{I}(\theta) \Lambda,
$$

$\Lambda$ encontrada em (4.8).

\subsubsection{Implementação computacional}

De posse das derivadas (de primeira e segunda ordens), dispomos de vários métodos iterativos de maximização do logaritmo da função verossimilhança. Estimativas baseadas nos estimadores consistentes da Seção 4.2 servem como valores iniciais dos esquemas iterativos. Optamos pela linguagem Ox (Doornik, 1998), que inclui implementações dos métodos BFGS, de Newton e do escore de Fisher. Os recursos matriciais da linguagem ajudaram na verificação de todos os cálculos de derivadas deste capítulo.

BFGS é um algoritmo que passo a passo constrói uma aproximação (definida negativa) da matriz de derivadas segundas de $\log \mathcal{L}(\theta ; \mathrm{Y}, \mathrm{X})$ (é um método quaseNewton). Detalhes podem ser vistos em Gill, Murray \& Wright (1981). Em Ox o vetor gradiente do logaritmo da função verossimilhança deve ser fornecido ou pode ser avaliado numericamente.

Os métodos de Newton e do escore de Fisher requerem derivadas de primeira ordem analíticas do logaritmo da função verossimilhança. Quanto às derivadas de segunda ordem, no método de Newton podem ser analíticas ou numéricas.

Nos modelos $34,38,42,46,50,54$ e 58 a distribuição conjunta de

$$
\left\{\left(Y_{i j}, X_{i j}, x_{i j}\right), j=1, \ldots, n, i=1, \ldots, k\right\}
$$

- dados completos segundo Dempster et al. (1977) - pertence à família exponencial regular, podendo simplificar o passo "M" do algoritmo EM, algo que comprovamos no modelo 34. Os demais modelos da Tabela 4.2 dependem de solução iterativa do passo "M", desestimulando o emprego deste algoritmo. Esboçamos na Figura 4.1 
um esquema do processo iterativo do algoritmo EM no modelo 34 . O passo "E" resume-se a calcular

$$
\widehat{x_{i j}}=\mathrm{E}\left(x_{i j} \mid \mathbf{Y}, \mathbf{X} ; \boldsymbol{\theta}\right) \text { e } \widehat{x_{i j}^{2}}=\mathrm{E}\left(x_{i j}^{2} \mid \mathbf{Y}, \mathbf{X} ; \boldsymbol{\theta}\right),
$$

que segue da distribuição conjunta.

$$
\left(\begin{array}{c}
Y_{i j} \\
X_{i j} \\
x_{i j}
\end{array}\right) \sim N_{3}\left(\left(\begin{array}{c}
\alpha+\beta \mu_{x i} \\
\mu_{x i} \\
\mu_{x i}
\end{array}\right),\left(\begin{array}{ccc}
\beta^{2} \sigma_{x x i}+\sigma_{e e} & \beta \sigma_{x x i} & \beta \sigma_{x x i} \\
& \sigma_{x x i}+\sigma_{u u} & \sigma_{x x i} \\
& & \sigma_{x x i}
\end{array}\right)\right),
$$

$j=1, \ldots, n, i=1, \ldots, k$.

Embora os valores iniciais dos parâmetros nos esquemas iterativos sejam baseados em estimadores consistentes, não há garantia de convergência para um ponto de máximo global do logaritmo da função verossimilhança.

\subsection{Resultados assintóticos}

Os modelos considerados neste capítulo, além de não exigirem suposições adicionais envolvendo os parâmetros, também permitem julgar a adequação do ajuste usando o teste da razão de verossimilhanças. Sejam

$$
\left(\begin{array}{l}
\mathbf{Y}_{j} \\
\mathbf{X}_{j}
\end{array}\right) \stackrel{\text { iid }}{\sim} \mathrm{N}_{2 k}\left(\boldsymbol{\mu}^{*}, \boldsymbol{\Sigma}^{*}\right)
$$

com $\mathrm{Y}_{j}$ e $\mathrm{X}_{j}$ trazidos de (4.4), $j=1, \ldots, n, \boldsymbol{\mu}^{*} \in \mathbb{R}^{2 k}, \boldsymbol{\Sigma}^{*}$ definida positiva dada por

$$
\Sigma^{*}=\left(\begin{array}{ll}
\mathbf{D}\left(\sigma_{Y Y 1}^{*}, \ldots, \sigma_{Y Y k}^{*}\right) & \mathbf{D}\left(\sigma_{Y X 1}^{*}, \ldots, \sigma_{Y X k}^{*}\right) \\
& \mathbf{D}\left(\sigma_{X X 1}^{*}, \ldots, \sigma_{X X k}^{*}\right)
\end{array}\right) .
$$

Livres de quaisquer outras restrições, os estimadores de MV de $\boldsymbol{\mu}^{*}$ e $\boldsymbol{\Sigma}^{*}$ são

$$
\widehat{\boldsymbol{\mu}^{*}}=\left(\begin{array}{l}
\overline{\mathbf{Y}} \\
\overline{\mathbf{X}}
\end{array}\right) \quad \text { e } \widehat{\boldsymbol{\Sigma}^{*}}=\frac{n-1}{n}\left(\begin{array}{ll}
\mathbf{D}\left(m_{Y Y 1}, \ldots, m_{Y Y k}\right) & \mathbf{D}\left(m_{Y X 1}, \ldots, m_{Y X k}\right) \\
& \mathbf{D}\left(m_{X X 1}, \ldots, m_{X X k}\right)
\end{array}\right),
$$

onde $m_{Y Y i}, m_{Y X i}$ e $m_{X X i}$ são as covariâncias amostrais, conforme (4.3), $i=1, \ldots, k$. O número total de parâmetros em $\boldsymbol{\mu}^{*}$ e $\Sigma^{*}$ é $5 k$. O logaritmo da função verossimilhança de (4.13) é indicado por $\log \mathcal{L}^{*}\left(\boldsymbol{\mu}^{*}, \Sigma^{*} ; \mathbf{Y}, \mathbf{X}\right)$. Sejam agora

$$
\left(\begin{array}{c}
\mathbf{Y}_{j} \\
\mathbf{X}_{j}
\end{array}\right) \stackrel{\text { iid }}{\sim} \mathrm{N}_{2 k}\left(\left(\begin{array}{c}
\boldsymbol{\mu}_{y} \\
\boldsymbol{\mu}_{x}
\end{array}\right), \mathrm{V}\right),
$$


início

estimativas iniciais:

$$
\begin{aligned}
& \widehat{\mu_{x i}}=\bar{X}_{i .}, i=1, \ldots, k, \\
& \widehat{\beta}=\frac{2}{k(k-1)} \sum_{i=1}^{k} \sum_{l=i+1}^{k} \frac{m_{Y X i}-m_{Y X l}}{m_{X X i}-m_{X X l}}, \\
& \widehat{\alpha}=\frac{1}{k} \sum_{i}\left(\bar{Y}_{i .}-\widehat{\beta} \bar{X}_{i .}\right), \\
& {\widehat{\sigma_{x x i}}}^{*}=m_{Y X i} / \widehat{\beta}, \quad \widehat{\sigma_{x x i}}={\widehat{\sigma_{x x i}}}^{*} I_{(0, \infty)}\left({\widehat{\sigma_{x x i}}}^{*}\right)+\epsilon I_{(-\infty, 0]}\left({\widehat{\sigma_{x x i}}}^{*}\right), i=1, \ldots, k \\
& {\widehat{\sigma_{u u}}}^{*}=\frac{1}{k} \sum_{i}\left(m_{X X i}-\frac{m_{Y X i}}{\widehat{\beta}}\right), \quad \widehat{\sigma_{u u}}={\widehat{\sigma_{u u}}}^{*} I_{(0, \infty)}\left({\widehat{\sigma_{u u}}}^{*}\right)+\epsilon I_{(-\infty, 0]}\left({\widehat{\sigma_{u u}}}^{*}\right), \\
& {\widehat{\sigma_{e e}}}^{*}=\frac{1}{k} \sum_{i}\left(m_{Y Y i}-\widehat{\beta} m_{Y X i}\right), \quad, \quad{\widehat{\sigma_{e e}}}={\widehat{\sigma_{e e}}}^{*} I_{(0, \infty)}\left({\widehat{\sigma_{e e}}}^{*}\right)+\epsilon I_{(-\infty, 0]}\left(\widehat{\sigma}_{e e}^{*}\right),
\end{aligned}
$$

passo E:

$$
\begin{aligned}
& \widehat{\delta_{i}}=\left(\widehat{\beta}_{i}^{2} \widehat{\sigma_{u u}}+\widehat{\sigma_{e e}}\right) \widehat{\sigma_{x x i}}+\widehat{\sigma_{u u}} \widehat{\sigma_{e e}}, \\
& \widehat{x_{i j}}=\widehat{\mu_{x i}}+\widehat{\sigma_{x x i}}\left[\widehat{\beta} \widehat{\sigma_{u u}}\left(Y_{i j}-\widehat{\alpha}-\widehat{\beta} \widehat{\mu_{x i}}\right)+\widehat{\sigma_{e e}}\left(X_{i j}-\widehat{\mu_{x i}}\right)\right] / \widehat{\delta_{i}}, \\
& \widehat{x_{i j}^{2}}=\widehat{\sigma_{u u}} \widehat{\sigma_{e e}} \widehat{\sigma_{x x i}} / \widehat{\delta_{i}}+\left(\widehat{x_{i j}}\right)^{2}, j=1, \ldots, n, i=1, \ldots, k,
\end{aligned}
$$

passo M:

$$
\begin{aligned}
& \widehat{\mu_{x i}}=\overline{\widehat{x_{i .}}}, i=1, \ldots, k, \\
& \widehat{\beta}=\frac{\frac{1}{n k} \sum_{i, j} Y_{i j} \widehat{x_{i j}}-\bar{Y} . . \widehat{\widehat{x_{.}}}}{\frac{1}{n k} \sum_{i, j} \widehat{x_{i j}^{2}}-\left(\overline{\widehat{x_{.}}}\right)^{2}}, \\
& \widehat{\alpha}=\bar{Y} . .-\widehat{\beta \widehat{x_{.}}}, \\
& \widehat{\sigma_{u u}}=\frac{1}{n k} \sum_{i, j}\left(X_{i j}-2 X_{i j} \widehat{x_{i j}}+\widehat{x_{i j}^{2}}\right), \\
& \widehat{\sigma_{x x i}}=\frac{1}{n} \sum_{j} \widehat{x_{i j}^{2}}-\left(\widehat{\widehat{x_{i}}}\right)^{2}, i=1, \ldots, k, \\
& \widehat{\sigma_{e e}}=\frac{1}{n k} \sum_{i, j} Y_{i j}^{2}-\frac{1}{k} \sum_{i}\left[\widehat{\beta}^{2} \widehat{\sigma_{x x i}}+\left(\widehat{\alpha}+\widehat{\beta} \widehat{x_{i .}}\right)^{2}\right],
\end{aligned}
$$

repetir passos $\mathrm{E}$ e $\mathrm{M}$, fim.

Figura 4.1: Algoritm̄8 EM no modelo 34. 
$j=1, \ldots, n$, onde $\boldsymbol{\mu}_{y}, \boldsymbol{\mu}_{x}$ e V (totalizando $p$ parâmetros) dependem do modelo, segundo exposto na Seção 4.2. Sob a hipótese nula de que a distribuição das observações segue (4.14), temos que a distribuição limite de

$$
w=2\left[\log \mathcal{L}^{*}\left(\widehat{\boldsymbol{\mu}^{*}}, \widehat{\boldsymbol{\Sigma}^{*}} ; \mathbf{Y}, \mathbf{X}\right)-\log \mathcal{L}(\widehat{\boldsymbol{\theta}} ; \mathbf{Y}, \mathbf{X})\right],
$$

quando $n \rightarrow \infty$, é qui-quadrado com $5 k-p$ graus de liberdade, se $5 k-p>0$, onde $\log \mathcal{L}(\boldsymbol{\theta} ; \mathbf{Y}, \mathbf{X})$ vem de (4.5). Assim, podemos testar o ajuste de qualquer um dos 37 modelos identificáveis da Tabela 4.2 e comparar modelos encaixados.

Verificado o ajuste satisfatório de um modelo, estimativas das variâncias dos estimadores de MV dos parâmetros podem ser obtidas com o auxílio de outro resultado assintótico, a saber:

$$
\sqrt{n}(\widehat{\boldsymbol{\theta}}-\boldsymbol{\theta}) \stackrel{\mathrm{d}}{\longrightarrow} \mathrm{N}_{p}\left(\mathbf{0},\left[\boldsymbol{\Lambda} \mathcal{I}_{1}(\boldsymbol{\theta}) \boldsymbol{\Lambda}^{\prime}\right]^{-1}\right)
$$

quando $n \rightarrow \infty, \operatorname{com} \Lambda$ e $\mathcal{I}_{1}(\theta)$ dadas por (4.8) e (4.11), respectivamente. Nos modelos 50, 52, 54, 57, 58 e 61 o estimador de MV de $\boldsymbol{\mu}_{x}$ é $\widehat{\boldsymbol{\mu}_{x}}=\overline{\mathbf{X}}$., de maneira que $\widehat{\mu_{x i}} \sim \mathrm{N}\left(\mu_{x i},\left(\sigma_{u u i}+\sigma_{x x i}\right) / n\right)$, podendo $\sigma_{u u i}$ e (ou) $\sigma_{x x i}$ serem constantes nos grupos (Tabela 4.1), $i=1, \ldots, k$.

\subsection{Influência local}

Nesta seção a técnica de influência local proposta por Cook (1986) é aplicada ao estudo das variações causadas por perturbações do modelo sobre os estimadores de MV dos parâmetros do modelo. Na Seção 3.4 apresentamos um resumo da proposta de Cook, bem como definições e notações que serão aproveitadas aqui. Em seguida fornecemos expressões para a matriz $\Delta$, com dimensão $6 k \times q$ (referente ao modelo 64), onde $q$ é a dimensão do vetor de perturbações. Em um modelo qualquer, de forma semelhante a outras matrizes deste capítulo, $\Delta$ deve ser multiplicada à direita por $\Lambda$ definida em (4.8). Adotaremos a partição

$$
\Delta=\left(\Delta_{\mu_{x}}^{\prime}, \Delta_{\alpha}^{\prime}, \Delta_{\beta}^{\prime}, \Delta_{\sigma_{u u}}^{\prime}, \Delta_{\sigma_{e e}}^{\prime}, \Delta_{\sigma_{x x}}^{\prime}\right)^{\prime}
$$

cada bloco tendo dimensão $k \times q$. Dois diferentes esquemas de perturbação serão tratados. 


\subsubsection{Perturbação das respostas}

As respostas $\mathbf{Y}_{j}=\left(Y_{1 j}, \ldots, Y_{k j}\right)^{\prime}$ são modificadas por um vetor $\omega_{j}=\left(w_{1 j}, \ldots, w_{k j}\right)^{\prime}$, $j=1, \ldots, n$, segundo esquemas aditivo e multiplicativo:

$$
\mathbf{Y}_{j}\left(\omega_{j}\right)= \begin{cases}\mathbf{Y}_{j}+\omega_{j}, & \text { para perturbações aditivas, } \\ \mathbf{Y}_{j} \odot \omega_{j}, & \text { para perturbações multiplicativas, }\end{cases}
$$

$j=1, \ldots, n$. Os vetores $\omega_{0}=0$ e $\omega_{0}=\mathbf{1}_{n k}$, respectivamente, reproduzem os modelos sem perturbação. As funções log-verossimilhança perturbadas seguem de (4.5) substituindo $\mathbf{Y}_{j}$ por $\mathbf{Y}_{j}\left(\boldsymbol{\omega}_{j}\right)$ na expressão de $\boldsymbol{d}_{j}, j=1, \ldots, n$. Sejam $\Delta^{\mathrm{a}}$ e $\Delta^{\mathrm{m}}$ as matrizes dos esquemas aditivo (a) e multiplicativo ( $\mathrm{m}$ ), subdivididas conforme (4.16). Antes de apresentarmos estes blocos necessitamos de algumas matrizes auxiliares. Definimos

$$
\Delta_{\mu_{x}}^{\mathrm{a} *}=\left(\Delta_{\mu_{x 1}}^{\mathrm{a} *}, \ldots, \Delta_{\mu_{x n}}^{\mathrm{a} *}\right) \quad \text { e } \quad \Delta_{\mu_{x}}^{\mathrm{m} *}=\left(\Delta_{\mu_{x 1}}^{\mathrm{m} *}, \ldots, \Delta_{\mu_{x n}}^{\mathrm{m} *}\right) ;
$$

as matrizes dos demais parâmetros são definidas de modo similar, dadas por

$$
\begin{gathered}
\Delta_{\mu_{x j}}^{\mathrm{a} *}=\beta \odot \sigma_{u u} \oslash \delta \quad, \quad \Delta_{\alpha_{j}}^{\mathrm{a} *}=\left(\sigma_{u u}+\sigma_{x x}\right) \oslash \delta \\
\Delta_{\beta_{j}}^{\mathrm{a} *}=\sigma_{x x} \odot\left(\beta \odot \boldsymbol{p}_{11 j}^{*}+p_{12 j}^{*}\right)+\mu_{x} \odot \Delta_{\alpha_{j}}^{\mathrm{a} *}, \quad \Delta_{\sigma_{u u j}}^{\mathrm{a} *}=\frac{1}{2} \boldsymbol{p}_{22 j}^{*} \quad, \quad \Delta_{\sigma_{e e j}}^{\mathrm{a} *}=\frac{1}{2} p_{11 j}^{*}, \\
\Delta_{\sigma_{x x j}}^{\mathrm{a} *}=\frac{1}{2}\left(\beta \odot \beta \odot \boldsymbol{p}_{11 j}^{*}+2 \beta \odot \boldsymbol{p}_{12 j}^{*}+\boldsymbol{p}_{22 j}^{*}\right)
\end{gathered}
$$

e

$$
\Delta_{\gamma_{j}}^{\mathrm{m} *}=\Delta_{\gamma_{j}}^{\mathrm{a} *} \odot \mathbf{Y}_{j} \quad, \quad \gamma \in\left\{\mu_{x}, \alpha, \beta, \sigma_{u u}, \sigma_{e e}, \sigma_{x x}\right\},
$$

onde

$$
\begin{aligned}
& \boldsymbol{p}_{11 j}^{*}= 2\left(\boldsymbol{\sigma}_{u u}+\boldsymbol{\sigma}_{x x}\right) \odot\left[\left(\boldsymbol{\sigma}_{u u}+\boldsymbol{\sigma}_{x x}\right) \odot\left(\mathbf{Y}_{j}-\boldsymbol{\alpha}-\boldsymbol{\beta} \odot \boldsymbol{\mu}_{x}\right)-\boldsymbol{\beta} \odot \boldsymbol{\sigma}_{x x} \odot\left(\mathbf{X}_{j}-\boldsymbol{\mu}_{x}\right)\right] \oslash(\boldsymbol{\delta} \odot \boldsymbol{\delta}), \\
& \boldsymbol{p}_{12 j}^{*}=\left\{-2\left(\boldsymbol{\sigma}_{u u}+\boldsymbol{\sigma}_{x x}\right) \odot \boldsymbol{\beta} \odot \boldsymbol{\sigma}_{x x} \odot\left(\mathbf{Y}_{j}-\boldsymbol{\alpha}-\boldsymbol{\beta} \odot \boldsymbol{\mu}_{x}\right)+\left[\boldsymbol{\beta} \odot \boldsymbol{\beta} \odot \boldsymbol{\sigma}_{x x} \odot \boldsymbol{\sigma}_{x x}\right.\right. \\
&\left.\left.+\left(\boldsymbol{\sigma}_{u u}+\boldsymbol{\sigma}_{x x}\right) \odot\left(\boldsymbol{\sigma}_{e e}+\boldsymbol{\beta} \odot \boldsymbol{\beta} \odot \boldsymbol{\sigma}_{x x}\right)\right] \odot\left(\mathbf{X}_{j}-\boldsymbol{\mu}_{x}\right)\right\} \oslash(\boldsymbol{\delta} \odot \boldsymbol{\delta}) \text { e } \\
& \boldsymbol{p}_{22 j}^{*}= 2 \boldsymbol{\beta} \odot \boldsymbol{\sigma}_{x x} \odot\left[\boldsymbol{\beta} \odot \boldsymbol{\sigma}_{x x} \odot\left(\mathbf{Y}_{j}-\boldsymbol{\alpha}-\boldsymbol{\beta} \odot \boldsymbol{\mu}_{x}\right)-\left(\boldsymbol{\sigma}_{e e}+\boldsymbol{\beta} \odot \boldsymbol{\beta} \odot \boldsymbol{\sigma}_{x x}\right) \odot\left(\mathbf{X}_{j}-\boldsymbol{\mu}_{x}\right)\right] \oslash(\boldsymbol{\delta} \odot \boldsymbol{\delta}), \\
& j=1, \ldots, n . \text { Finalmente, fazemos }
\end{aligned}
$$

$$
\Delta_{\mu_{x}}^{\mathrm{a}}=\operatorname{bdiag}\left(\Delta_{\mu_{x}, 1}^{\mathrm{a} *}, \ldots, \Delta_{\mu_{x}, k}^{\mathrm{a} *}\right),
$$

onde $\Delta_{\mu_{x}, i}^{\mathrm{a} *}$ é a $i$-ésima linha de $\Delta_{\mu_{x}}^{\mathrm{a} *}, i=1, \ldots, k$. Os blocos restantes de $\Delta^{\mathrm{a}}$ e $\Delta^{\mathrm{m}}$ são processados analogamente. 


\subsubsection{Perturbação das covariáveis}

As covariáveis $\mathbf{X}_{j}=\left(X_{1 j}, \ldots, X_{k j}\right)^{\prime}$ são alteradas por um vetor $\omega_{j}=\left(w_{1 j}, \ldots, w_{k j}\right)^{\prime}$, $j=1, \ldots, n$, de acordo com esquemas aditivo e multiplicativo,

$$
\mathbf{X}_{j}\left(\omega_{j}\right)= \begin{cases}\mathbf{X}_{j}+\omega_{j}, & \text { para perturbações aditivas, } \\ \mathbf{X}_{j} \odot \omega_{j}, & \text { para perturbações multiplicativas }\end{cases}
$$

$j=1, \ldots, n$. Expressões dos logaritmos das funções verossimilhança perturbadas decorrem de (4.5) ao substituirmos $\mathbf{X}_{j}$ por $\mathbf{X}_{j}\left(\omega_{j}\right)$ em $\boldsymbol{d}_{j}, j=1, \ldots, n$. Mantendo notações da Seção 4.4.1, apresentamos abaixo as matrizes auxiliares $\left(\Delta_{\mu_{x}}^{\mathrm{a} *}, \Delta_{\mu_{x}}^{\mathrm{m} *}, \ldots\right)$ :

$$
\begin{gathered}
\Delta_{\mu_{x j}}^{\mathrm{a} *}=\sigma_{e e} \oslash \delta, \quad \Delta_{\alpha_{j}}^{\mathrm{a} *}=-\beta \odot \boldsymbol{\sigma}_{x x} \oslash \boldsymbol{\delta}, \\
\Delta_{\beta_{j}}^{\mathrm{a} *}=\sigma_{x x} \odot\left(\beta \odot \boldsymbol{p}_{11 j}^{* *}+\boldsymbol{p}_{12 j}^{* *}\right)+\boldsymbol{\mu}_{x} \odot \Delta_{\alpha_{j}}^{\mathrm{a} *}, \quad \Delta_{\sigma_{u u j}}^{\mathrm{a} *}=\frac{1}{2} p_{22 j}^{* *}, \quad \Delta_{\sigma_{e e j}}^{\mathrm{a} *}=\frac{1}{2} p_{11 j}^{* *}, \\
\Delta_{\sigma_{x x j}}^{\mathrm{a} *}=\frac{1}{2}\left(\boldsymbol{\beta} \odot \beta \odot \boldsymbol{p}_{11 j}^{* *}+2 \beta \odot \boldsymbol{p}_{12 j}^{* *}+\boldsymbol{p}_{22 j}^{* *}\right)
\end{gathered}
$$

e

$$
\Delta_{\gamma_{j}}^{\mathrm{m} *}=\Delta_{\gamma_{j}}^{\mathrm{a} *} \odot \mathbf{X}_{j} \quad, \quad \gamma \in\left\{\mu_{x}, \alpha, \beta, \sigma_{u u}, \sigma_{e e}, \sigma_{x x}\right\}
$$

onde

$$
\begin{aligned}
\boldsymbol{p}_{11 j}^{* *}= & -2 \odot \boldsymbol{\beta} \odot \boldsymbol{\sigma}_{x x} \odot\left[\left(\boldsymbol{\sigma}_{u u}+\boldsymbol{\sigma}_{x x}\right) \odot\left(\mathbf{Y}_{j}-\boldsymbol{\alpha}-\boldsymbol{\beta} \odot \boldsymbol{\mu}_{x}\right)-\boldsymbol{\beta} \odot \boldsymbol{\sigma}_{x x} \odot\left(\mathbf{X}_{j}-\boldsymbol{\mu}_{x}\right)\right] \oslash(\boldsymbol{\delta} \odot \boldsymbol{\delta}), \\
\boldsymbol{p}_{12 j}^{* *}= & \left\{\left[\boldsymbol{\beta} \odot \boldsymbol{\beta} \odot \boldsymbol{\sigma}_{x x} \odot \boldsymbol{\sigma}_{x x}+\left(\boldsymbol{\sigma}_{u u}+\boldsymbol{\sigma}_{x x}\right) \odot\left(\boldsymbol{\sigma}_{e e}+\boldsymbol{\beta} \odot \boldsymbol{\beta} \odot \boldsymbol{\sigma}_{x x}\right)\right] \odot\left(\mathbf{Y}_{j}-\boldsymbol{\alpha}-\boldsymbol{\beta} \odot \boldsymbol{\mu}_{x}\right)\right. \\
& \left.-2 \boldsymbol{\beta} \odot \boldsymbol{\sigma}_{x x} \odot\left(\boldsymbol{\sigma}_{e e}+\boldsymbol{\beta} \odot \boldsymbol{\beta} \odot \boldsymbol{\sigma}_{x x}\right) \odot\left(\mathbf{X}_{j}-\boldsymbol{\mu}_{x}\right)\right\} \oslash(\boldsymbol{\delta} \odot \boldsymbol{\delta}) \mathrm{e} \\
\boldsymbol{p}_{22 j}^{* *}= & 2 \odot\left(\boldsymbol{\sigma}_{e e}+\boldsymbol{\beta} \odot \boldsymbol{\beta} \odot \boldsymbol{\sigma}_{x x}\right) \odot\left[-\boldsymbol{\beta} \odot \boldsymbol{\sigma}_{x x} \odot\left(\mathbf{Y}_{j}-\boldsymbol{\alpha}-\boldsymbol{\beta} \odot \boldsymbol{\mu}_{x}\right)\right. \\
& \left.+\left(\boldsymbol{\sigma}_{e e}+\boldsymbol{\beta} \odot \boldsymbol{\beta} \odot \boldsymbol{\sigma}_{x x}\right) \odot\left(\mathbf{X}_{j}-\boldsymbol{\mu}_{x}\right)\right] \oslash(\boldsymbol{\delta} \odot \boldsymbol{\delta}),
\end{aligned}
$$

$j=1, \ldots, n$. Os blocos de $\Delta^{\mathrm{a}}$ e $\Delta^{\mathrm{m}}$ (4.16) são compostos seguindo o que foi estabelecido em (4.17).

Se pretendemos perturbar apenas valores das covariáveis do $i$-ésimo grupo, $i=$ $1, \ldots, k$, temos

$$
\mathbf{X}_{j}\left(\omega_{j}\right)=\left(X_{1 j}, \ldots, X_{i j}\left(\omega_{j}\right), \ldots, X_{k j}\right)^{\prime}
$$


onde

$$
X_{i j}\left(\omega_{j}\right)= \begin{cases}X_{i j}+\omega_{j}, & \text { para perturbações aditivas, } \\ X_{i j} \omega_{j}, & \text { para perturbações multiplicativas, }\end{cases}
$$

$j=1, \ldots, n$, notando que $\omega_{0}=0$ e $\omega_{0}=\mathbf{1}_{n}$ significam nenhuma perturbação. As matrizes $\Delta^{\mathrm{a}}$ e $\Delta^{\mathrm{m}}$ (esquemas aditivo e multiplicativo) são calculadas com base nas matrizes auxiliares da p. 81. Por exemplo,

$$
\Delta_{\mu_{x}}^{\mathrm{a}}=\left(\begin{array}{c}
0 \\
\vdots \\
\Delta_{\mu_{x}, i}^{\mathrm{a} *} \\
\vdots \\
0
\end{array}\right) \quad \text { e } \quad \Delta_{\mu_{x}}^{\mathrm{m}}=\left(\begin{array}{c}
0 \\
\vdots \\
\Delta_{\mu_{x}, i}^{\mathrm{m} *} \\
\vdots \\
0
\end{array}\right)
$$

onde $\Delta_{\mu_{x}, i}^{\mathrm{a} *}$ é a $i$-ésima linha de $\Delta_{\mu_{x}}^{\mathrm{a} *}, i=1, \ldots, k$. De forma similar tratamos as perturbações nas respostas no $i$-ésimo grupo,

$$
\mathbf{Y}_{j}\left(\omega_{j}\right)=\left(Y_{1 j}, \ldots, Y_{i j}\left(\omega_{j}\right), \ldots, Y_{k j}\right)^{\prime}
$$

onde

$$
Y_{i j}\left(\omega_{j}\right)= \begin{cases}Y_{i j}+\omega_{j}, & \text { para perturbações aditivas, } \\ Y_{i j} \omega_{j}, & \text { para perturbações multiplicativas }\end{cases}
$$

$j=1, \ldots, n$.

Em todos os esquemas de perturbação as matrizes $\Lambda \Delta$ são avaliadas nas estimativas de MV de $\theta, \operatorname{com} \boldsymbol{\Lambda}$ definida em (4.8). Recordando a Seção 3.4, a análise de autovalores e autovetores é efetuada com a matriz

$$
\Delta^{\prime} \Lambda^{\prime}\left[\Lambda \frac{\partial^{2}}{\partial \theta \partial \theta^{\prime}} \log \mathcal{L}(\boldsymbol{\theta} ; \mathbf{Y}, \mathbf{X}) \Lambda^{\prime}\right]^{-1} \Lambda \Delta
$$

notando que a matriz de derivadas de segunda ordem de $\log \mathcal{L}$ foi calculada na Seção 4.2.1.

\subsection{Aplicação}

Desenvolvemos nesta seção um exemplo de aplicação. 


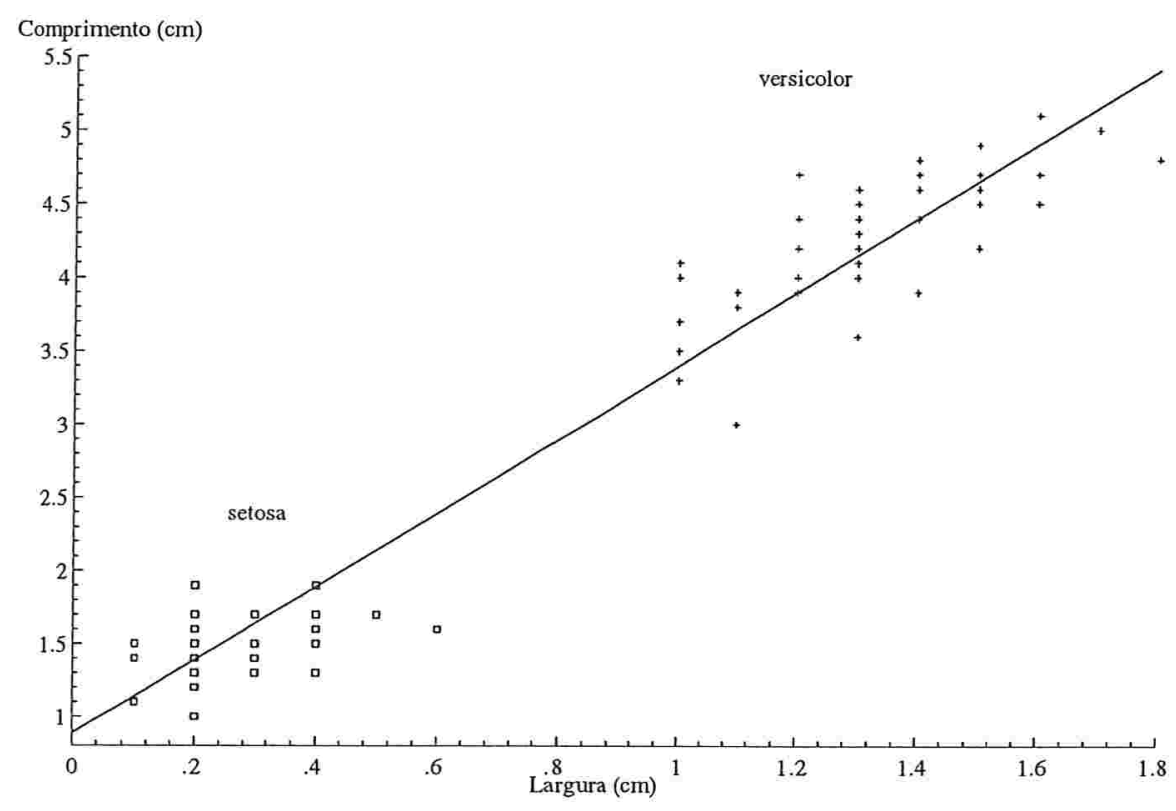

Figura 4.2: Gráfico de dispersão da largura e do comprimento de pétala e reta ajustada por mínimos quadrados - Exemplo 4.1.

Exemplo 4.1 Selecionamos 50 pares de medições de largura de pétala (covariável) e comprimento de pétala (variável resposta), ambas em centímetros, realizadas em flores das espécies Iris setosa e Iris versicolor (grupos) supondo que houve erro de medição. Estes dados são parte do conjunto utilizado por R. A. Fisher em Análise Discriminante (Kendall, Stuart \& Ord, 1983). A Figura 4.2 apresenta o gráfico de dispersão das variáveis, bem como a reta ajustada por mínimos quadrados ordinários, fornecendo estimativas $\widehat{\alpha}=0,88669$ e $\widehat{\beta}=2,5118$. Consideramos que os erros de medição têm variâncias $\left(\sigma_{u u}\right.$ e $\left.\sigma_{e e}\right)$ homogêneas nos dois grupos. A Figura 4.2 sugere diferenças entre as médias $\left(\mu_{x}\right)$ e também entre as variâncias $\left(\sigma_{x x}\right)$ das larguras. Restando os interceptos $(\alpha)$ e os coeficientes da covariável $(\beta)$, o modelo mais geral seria o modelo 58 (Tabela 4.1), que admite $\alpha$ e $\beta$ heterogêneos, mas com nenhum grau de liberdade, impedindo a rẹalização de um teste de ajuste. Na Tabela 4.1 o próximo candidato é o modelo 50, com vetor paramétrico $\theta=\left(\mu_{x 1}, \mu_{x 2}, \alpha_{1}, \alpha_{2}, \beta, \sigma_{u u}, \sigma_{e e}, \sigma_{x x 1}, \sigma_{x x 2}\right)^{\prime}$, totalizando nove parâmetros. O ajuste é aceitável, pois $w$ em (4.15) vale 1,5143 com nível descritivo igual a 0,21849 (um grau de liberdade). Simplificando, passamos ao modelo 34 , onde temos um só intercepto, $\theta=\left(\mu_{x 1}, \mu_{x 2}, \alpha, \beta, \sigma_{u u}, \sigma_{e e}, \sigma_{x x 1}, \sigma_{x x 2}\right)^{\prime}$ e o número de parâmetros é sete. O valor da estatística de ajuste ( $w$ ) é 1,7859, 
com dois graus de liberdade. Comparando os modelos 50 e 34, temos $w=0,2716$ a um nível descritivo de 0,60226 (um grau de liberdade), favorecendo o modelo mais simples. Tentamos ainda o modelo 33 (em que apenas $\mu_{x}$ difere nos dois grupos), mas resultou em um ajuste insatisfatório, com $w=45,126$ excessivamente grande para um grau de liberdade. Adotamos, portanto, o modelo 34. As estimativas dos parâmetros (acompanhadas de estimativas dos desvios padrão) são dadas por $\widehat{\mu_{x 1}}=0,24682(0,021816), \widehat{\mu_{x 2}}=1,3252(0,039399), \widehat{\alpha}=0,82073(0,073024)$, $\widehat{\beta}=2,5958(0,076179), \widehat{\sigma_{u u}}=0,010222(0,0024553), \widehat{\sigma_{e e}}=0,0098789(0,018487)$, $\widehat{\sigma_{x x 1}}=0,0018153(0,0014358)$ e $\widehat{\sigma_{x x 2}}=0,028724(0,0088697)$. As estimativas de $\mu_{x 1}$ e $\mu_{x 2}$ estão próximas das médias amostrais das covariáveis nos grupos $(0,246$ e 1,326), assim como as estimativas de $\alpha$ e $\beta$ estão próximas das estimativas de mínimos quadrados. Nos modelos 50 e 34 os métodos BFGS, de Newton e do escore de Fisher forneceram praticamente as mesmas estimativas de $\theta$. Experiências com derivadas analíticas e numéricas não produziram diferenças consideráveis nas estimativas dos parâmetros.

Perturbando as respostas (Seção 4.4.1), a Figura 4.3 (a) mostra que no esquema aditivo predominam no vetor $\boldsymbol{l}_{\max }$ componentes devidos à espécie Iris setosa (índices de 1 a 50), responsável pelos menores valores de comprimentos de pétala. Perturbações multiplicativas (Figura 4.3 (b)) têm efeito inverso (maiores valores de $\left|l_{\max }\right|$ devidos à espécie Iris versicolor), embora sem ser tão marcante como no caso anterior. Se estivermos interessados em $\theta_{1}=(\alpha, \beta)^{\prime}$, perturbações aditivas de sinais opostos nas medições dos comprimentos nos dois grupos provocam as maiores variações nas estimativas de $\theta_{1}$ (Figura 4.4 (a)). A Figura 4.4 (b) revela que para perturbações multiplicativas, nitidamente só é relevante o grupo Iris versicolor.

No esquema de perturbação aditiva dos valores das covariáveis (Seção 4.4.2), a Figura 4.5 (a) indica comportamento parecido nos dois grupos. Nas perturbações multiplicativas (Figura 4.5 (b)) os componentes do grupo Iris versicolor no vetor $\left|l_{\max }\right|$ se destacam. Se o interesse recair sobre $\theta_{1}=(\alpha, \beta)^{\prime}$, perturbações nas covariáveis (Figura 4.6) e nas respostas (Figura 4.4) afetam as estimativas de MV de $\theta_{1}$ de forma análoga. 


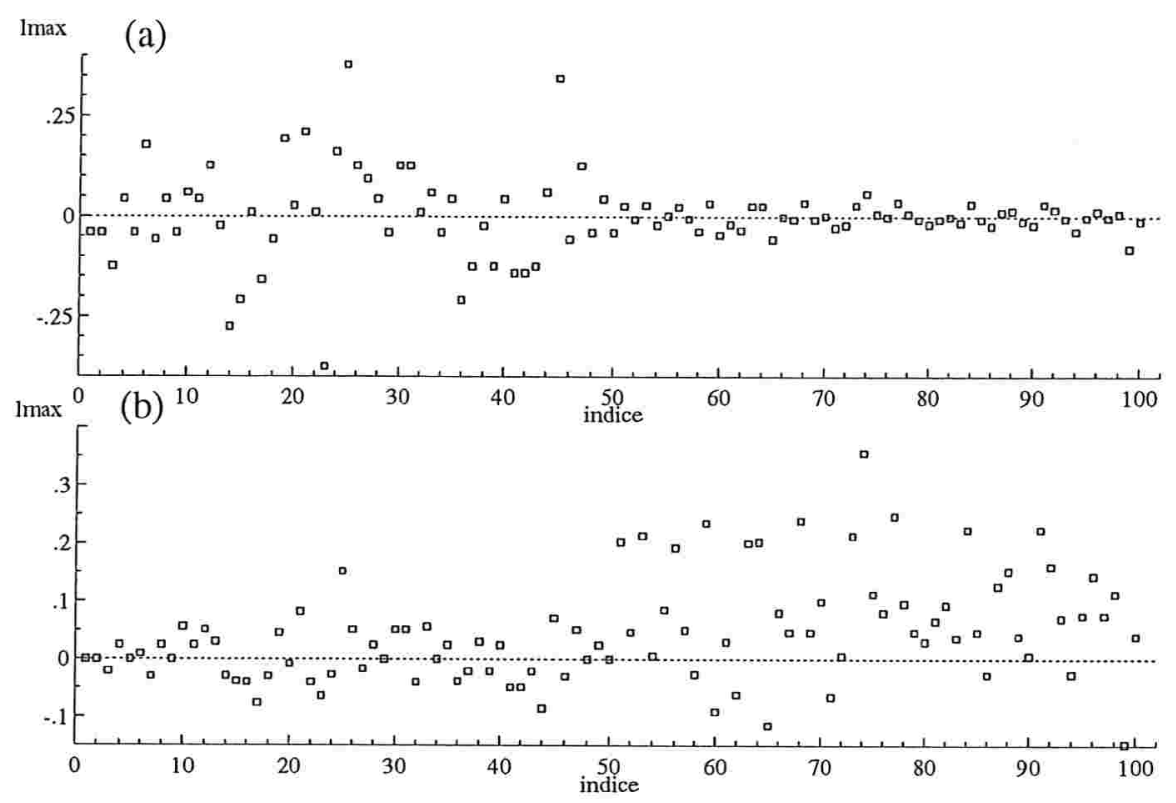

Figura 4.3: Gráfico de índices de $\boldsymbol{l}_{\max }$ nas perturbações aditiva (a) e multiplicativa (b) das respostas, interesse em $\theta$ - Exemplo 4.1 .

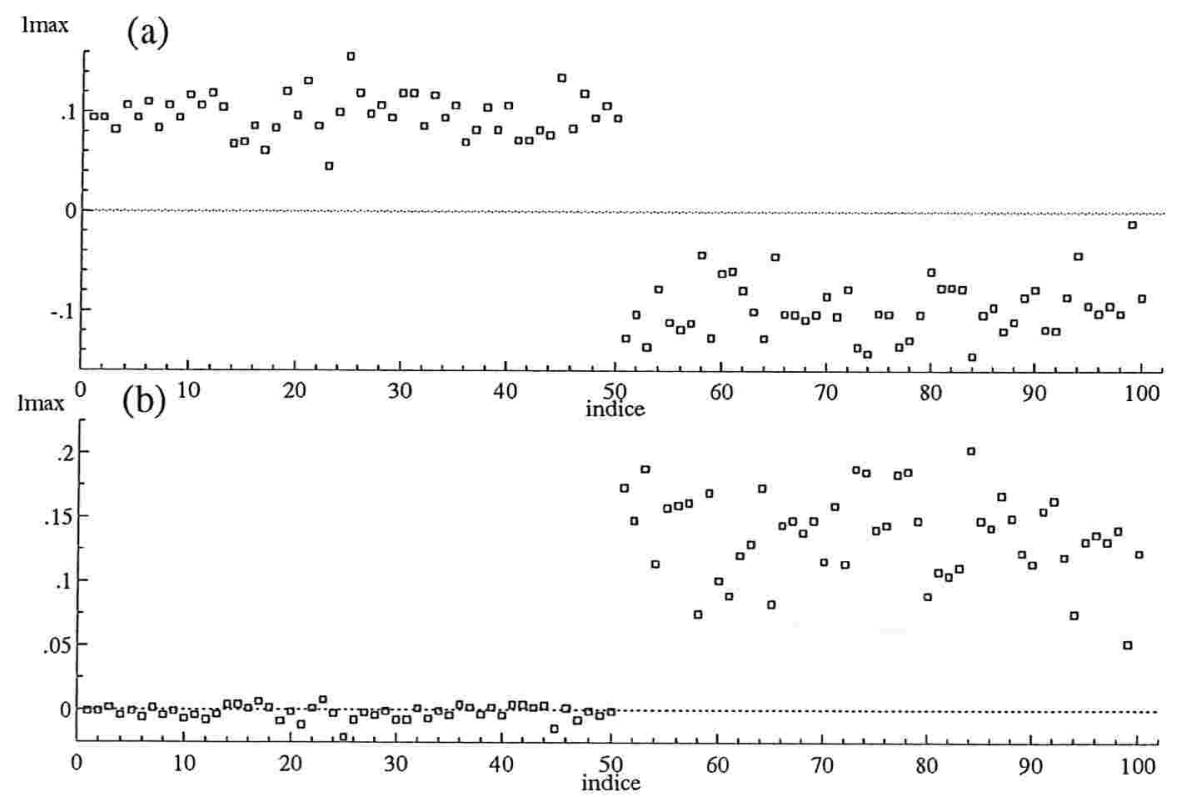

Figura 4.4: Gráfico de índices de $\boldsymbol{l}_{\max }$ nas perturbações aditiva (a) e multiplicativa (b) das respostas, interesse em $\theta_{1}$ - Exemplo 4.1 . 


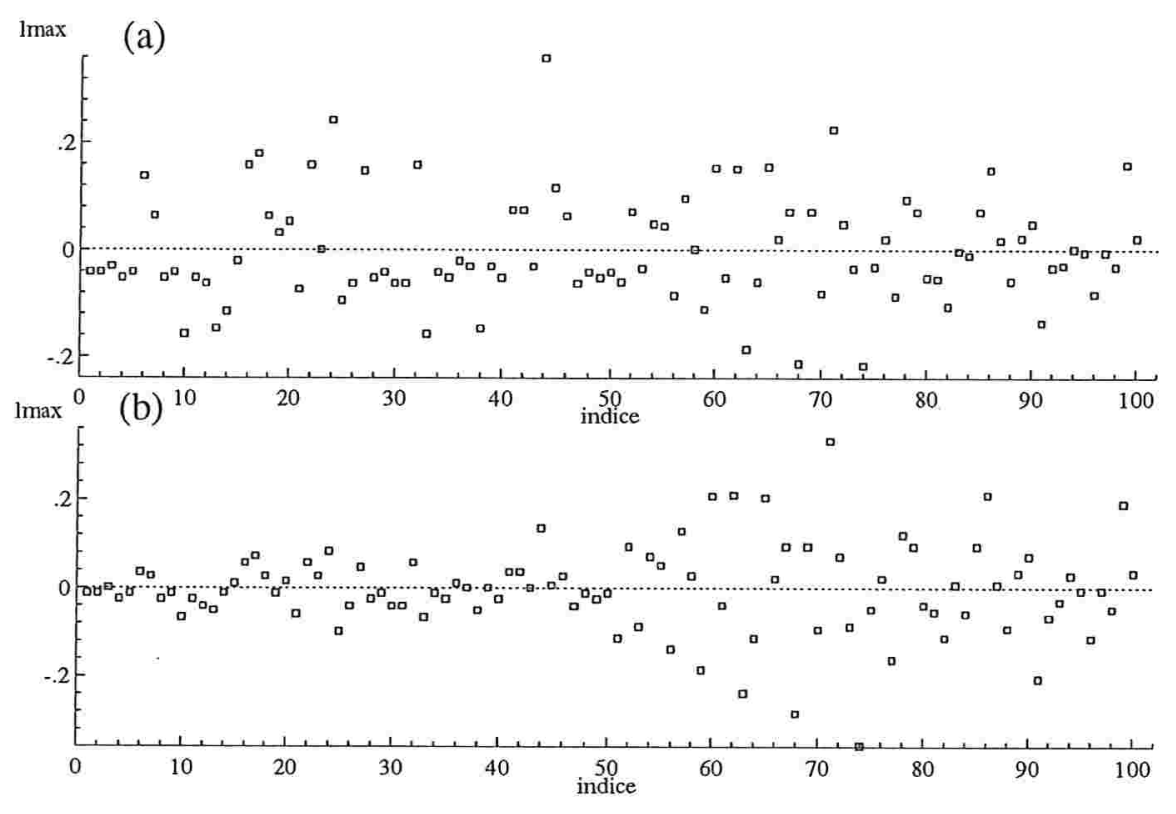

Figura 4.5: Gráfico de índices de $\boldsymbol{l}_{\max }$ nas perturbações aditiva (a) e multiplicativa (b) das covariáveis, interesse em $\theta$ - Exemplo 4.1.

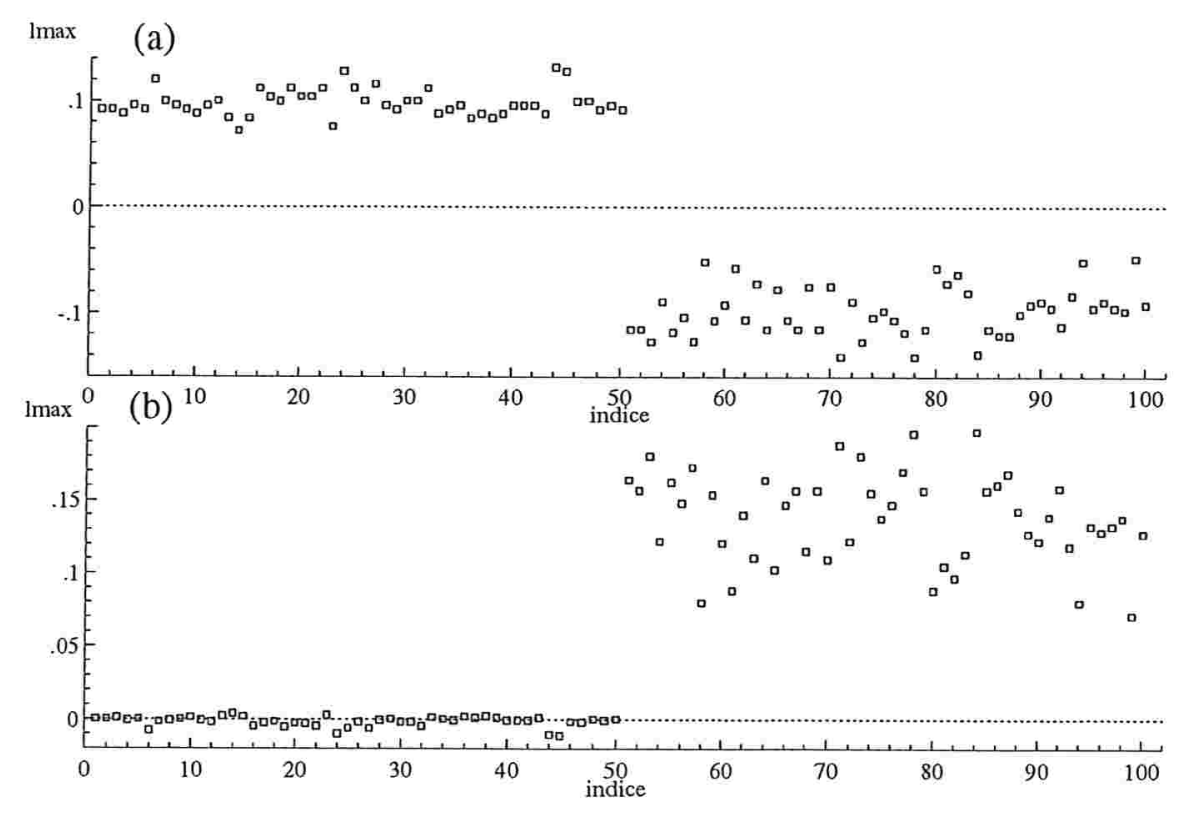

Figura 4.6: Gráfico de índices de $\boldsymbol{l}_{\max }$ nas perturbações aditiva (a) e multiplicativa (b) das covariáveis, interesse em $\theta_{1}$ - Exemplo 4.1. 


\subsection{Comentários finais}

Concluímos listando alguns itens que poderão ser detalhados futuramente. O logaritmo da função verossimilhança deve ser maximizado com restrições que garantam estimativas pertencentes ao espaço paramétrico. Testes de hipóteses relevantes e modelos desbalanceados no número de observações em cada população não foram considerados. O procedimento sugerido por Fuller (1987, Seção 4.3.2) pode ser empregado na estimação dos parâmetros dos modelos 50, 52, 54, 57, 58 e 61, pois nestes os estimadores de $\mathrm{MV}$ de $\mu_{Y i}=\mathrm{E}\left(Y_{i j}\right)$ e $\mu_{x i}=\mathrm{E}\left(X_{i j}\right)$ são tais que $\widehat{\mu_{Y}} i=\bar{Y}_{i \text {. }}$ e $\widehat{\mu_{x i}}=\bar{X}_{i,}, i=1, \ldots, k$ (são modelos de Análise Fatorial).

Acreditamos que os resultados que desenvolvemos, aplicáveis a 37 modelos diferentes, facilitarão extensões e complementos do nosso trabalho. 


\section{Referências Bibliográficas}

Barnett, V. D. (1969). Simultaneous Pairwise Linear Structural Relationships. Biometrics, 25(1), 129-142.

Bolfarine, H. \& de Castro, M. (2000). ANOCOVA Models with Measurement Errors. Statistics 83 Probability Letters, 50, 257-263.

Bolfarine, H. \& Galea-Rojas, M. (1995). Structural Comparative Calibration Using the EM Algorithm. Journal of Applied Statistics, 22(2), 277-292.

Brown, G. H. (1978). Generalized Least Squares Applied to the Linear Ultrastructural Model. Biometrika, 65(2), 441-444.

Brown, P. J. \& Fuller, W. A., editors (1990). Statistical Analysis of Measurement Error Models and Applications, volume 112 of Contemporary Mathematics, Providence, RI. American Mathematical Society.

Carroll, R. J. (1989). Covariance Analysis in Generalized Linear Measurement Error Models. Statistics in Medicine, 8, 1075-1093.

Carroll, R. J., Gallo, P. P. \& Gleser, L. J. (1985). Comparison of Least Squares and Errorsin-Variables Regression, With Special Reference to Randomized Analysis of Covariance. J. Amer. Statist. Assoc, 80(392), 929-932.

Carroll, R. J., Ruppert, D. \& Stefanski, L. A. (1995). Measurement Error in Nonlinear Models. Chapman \& Hall, New York.

Carter, R. L. (1981). Restricted Maximum-Likelihood Estimation of Bias and Reliability in the Comparison of Several Measuring Methods. Biometrics, 37(4), 733-741.

Chen, C.-C. (1997). Rank Transformations When Covariables are Measured With Error or Mismodelled. Comm. in Statist. - Theory and Methods, 26(12), 2967-2982.

Cheng, C.-L. \& Van Ness, J. W. (1999). Statistical Regression With Measurement Error, volume 6 of Kendall's Library of Statistics. Arnold, London. 
Chipkevitch, E., Nishimura, R. T., Tu, D. G. S. \& Galea-Rojas, M. (1996). Clinical Measurement of Testicular Volume in Adolescents: Comparison of the Reliability of 5 Methods. Journal of Urology, 156(6), 2050-2053.

Cochran, W. (1957). Analysis of Covariance : Its Nature and Uses. Biometrics, 13(3), 261-281.

Cochran, W. (1968). Errors of Measurement in Statistics. Technometrics, 10, 637-666.

Cook, R. D. (1986). Assessment of Local Influence (with Discussion). Journal of the Royal Statistical Society - Series B , 48(2), 133-169.

Cook, R. D. (1987). Influence Assessment. Journal of Applied Statistics, 14(2), 117-131.

Cox, D. R. \& McCullagh, P. (1982). Some Aspects of Analysis of Covariance. Biometrics, 38(3), 541-561.

Cox, N. R. (1976). The Linear Structural Relation for Several Groups of Data. Biometrika, 63(2), 231-237.

de Souza, F. A. M. (1999). Influência Local e Análise de Resíduos em Modelos de Regressão von Mises. Tese de doutorado, IME - Universidade de São Paulo, São Paulo.

DeGracie, J. (1968). Analysis of Covariance When the Concomitant Variable is Measured With Error. Ph.D. thesis, Iowa State University, Ames, Iowa.

DeGracie, J. \& Fuller, W. A. (1972). Estimation of the Slope and Analysis of Covariance when the Concomitant Variable is Measured With Error. J. Amer. Statist. Assoc., 67(340), 930-937.

Dempster, A. P., Laird, N. M. \& Rubin, D. B. (1977). Maximum Likelihood from Incomplete Data via the EM Algorithm (with Discussion). J. Roy. Statist. Soc. Ser. B, 39(1), $1-38$.

Dolby, G. R. (1976). The Ultrastructural Relation: A Synthesis of the Functional and Structural Relations. Biometrika, 63(1), 39-50.

Doornik, J. A. (1998). Object-Oriented Matrix Programming using Ox 2.0. Timberlake Consultants, London.

Dunn, G. (1989). Design and Analysis of Reliability Studies - The Statistical Evaluation of Measurement Errors. Arnold, London.

Fuller, W. A. (1987). Measurement Error Models. Wiley, New York. 
Galea-Rojas, M. (1995). Calibração Comparativa Estrutural e Funcional. Tese de doutorado, IME - Universidade de São Paulo, São Paulo.

Galea-Rojas, M., Bolfarine, H. \& de Castro, M. (1999). Local Influence in Comparative Calibration Models. Technical Report RT-MAE-9925, IME-USP, São Paulo. To appear in Biometrical Journal (2001).

Galea-Rojas, M., Vilca-Labra, F. \& Bolfarine, H. (2001). Local Influence in Elliptical Comparative Calibration Models. Technical Report in progress, IME-USP, São Paulo.

Gill, P. E., Murray, W. \& Wright, M. H. (1981). Practical Optimization. Academic Press, London.

Gleser, L. J. (1981). Estimation in a Multivariate "Errors in Variables" Regression Model: Large Sample Results. The Annals of Statistics, 9(1), 24-44.

Gleser, L. J. (1992). The Importance of Assessing Measurement Reliability in Multivariate Regression. J. Amer. Statist. Assoc., 87(419), 696-707.

Gleser, L. J. (1993). Estimators of Slopes in Linear Errors-in-Variables Regression-Models when the Predictors Have Known Reliability Matrix. Statistics \& Probability Letters, $17(2), 113-121$.

Gleser, L. J., Carroll, R. J. \& Gallo, P. P. (1987). The Limiting Distribution of Least Squares in an Errors-in-Variables Regression Model. The Annals of Statistics, 15(1), 220-233.

Grubbs, F. E. (1948). On Estimating Precision of Measuring Instruments and Product Variability. J. Amer. Statist. Assoc., 43(242), 243-264.

Grubbs, F. E. (1973). Errors of Measurement, Precision, Accuracy and Statistical Comparison of Measuring-Instruments. Technometrics, 15(1), 53-66.

Hwang, J. T. (1986). Multiplicative Errors-in-Variables Models With Applications to Recent Data Released by the U.S. Department of Energy. J. Amer. Statist. Assoc., 81(395), $680-688$.

Jaech, J. L. (1985). Statistical Analysis of Measurement Errors. Wiley, New York.

Kelly, G. (1984). The Influence Function in the Errors in Variables Problem. The Annals of Statistics, 12(1), 87-100.

Kelly, G. (1985). Use of the Structural Equations Model in Assessing the Reliability of a new Measurement Technique. Applied Statistics, 34, 258-263. 
Kendall, M. G., Stuart, A. S. \& Ord, J. K. (1983). The Advanced Theory of Statistics, volume 3. Griffin, London, fourth edition.

Kimura, D. K. (1992). Functional Comparative Calibration Using an EM Algorithm. Biometrics, 48(4), 1263-1271.

Kwan, C. W. \& Fung, W. K. (1998). Assessing Local Influence for Specific Restricted Likelihood: Application to Factor Analysis. Psychometrika, 63(1), 35-46.

Lehmann, E. L. (1997). Theory of Point Estimation. Springer-Verlag, New York.

Lord, F. M. (1960). Large-Sample Covariance Analysis When the Control Variable is Fallible. J. Amer. Statist. Assoc., 55, 307-321.

Muirhead, R. J. (1982). Aspects of Multivariate Statistical Theory. Wiley, New York.

Porter, A. C. (1967). The Effects of Using Fallible Variables in the Analysis of Covariance. Ph.D. thesis, University of Wisconsin, Madison, Wisconsin.

Searle, S. R. (1997). Linear Models. Wiley, New York. Wiley Classics Library.

Sen, P. K. \& Singer, J. M. (1993). Large Sample Methods in Statistics : An Introduction With Applications. Chapman \& Hall, New York.

Shyr, J. Y. (1984). Comparative Precision in Linear Structural Relationship. Technical Report 84-30, Purdue University, West Lafayette, IN.

Styan, G. (1973). Hadamard Products and Multivariate Statistical Analysis. Linear Algebra and Its Applications, 6, 217-240.

Theobald, C. M. \& Mallinson, J. R. (1978). Comparative Calibration, Linear Structural Relationships and Congeneric Measurements. Biometrics, 34(1), 39-45.

Van Huffel, S., editor (1997). Recent Advances in Total Least Squares Techniques and Errors-in-Variables Modeling, SIAM Proceedings Series List, Philadelphia, PA. SIAM.

Van Huffel, S. \& Vandewalle, J. (1991). The Total Least Squares Problems - Computational Aspects and Analysis, volume 9 of Frontiers in Applied Mathematics. SIAM, Philadelphia, PA.

Wong, M. Y. (1991). Bartlett Adjustment to the Likelihood Ratio Statistic for Testing Several Slopes. Biometrika, 78(1), 221-224. 
Wu, X. \& Luo, Z. (1993). Second-order Approach to Local Influence. Journal of the Royal Statistical Society - Series B, 55(4), 929-936.

Zhao, Y. \& Lee, A. H. (1998). Influence Diagnostics for Simultaneous Equations Models. Austral. E New Zealand J. Statist., 40(3), 345-357. 